University of Massachusetts Amherst

ScholarWorks@UMass Amherst

July 2019

\title{
THE BIOACTIVE EFFECTS OF CHICORIC ACID AS A FUNCTIONAL FOOD INGREDIENT
}

\author{
Ye Peng \\ University of Massachusetts Amherst
}

Follow this and additional works at: https://scholarworks.umass.edu/dissertations_2

Part of the Food Biotechnology Commons, and the Food Chemistry Commons

\section{Recommended Citation}

Peng, Ye, "THE BIOACTIVE EFFECTS OF CHICORIC ACID AS A FUNCTIONAL FOOD INGREDIENT" (2019). Doctoral Dissertations. 1588.

https://doi.org/10.7275/14153010 https://scholarworks.umass.edu/dissertations_2/1588

This Open Access Dissertation is brought to you for free and open access by the Dissertations and Theses at ScholarWorks@UMass Amherst. It has been accepted for inclusion in Doctoral Dissertations by an authorized administrator of ScholarWorks@UMass Amherst. For more information, please contact scholarworks@library.umass.edu. 


\title{
THE BIOACTIVE EFFECTS OF CHICORIC ACID AS A FUNCTIONAL FOOD INGREDIENT
}

\author{
A Dissertation Presented
}

by

YE PENG

\author{
Submitted to the Graduate School of the \\ University of Massachusetts Amherst in partial fulfillment \\ of the requirements for the degree of
}

DOCTOR OF PHILOSOPHY

MAY 2019

The Department of Food Science 
(C) Copyright by Ye Peng 2019

All Rights Reserved 


\title{
THE BIOACTIVE EFFECTS OF CHICORIC ACID AS A FUNCTIONAL FOOD INGREDIENT
}

\author{
A Dissertation Presented \\ by \\ YE PENG
}

Approved as to style and content by:

Yeonhwa Park, Chair

Lynne A. McLandsborough, Member

Young-Cheul Kim, Member

Eric A. Decker, Department Head

Department of Food Science 


\section{DEDICATIONS}

Dedicated to my husband, my parents, my twin sister, and parents-in-law. 


\section{ACKNOWLEDGEMENTS}

First of all, I would like to express the deepest gratitude to my advisor, Dr.

Yeonhwa Park, for giving me a great opportunity to pursue my Ph.D. study in her lab and for her continuous guidance, support, and patience during the four years. Without her kind help and encouragement, I cannot make it here. My sincere thanks also go to my thesis committee, Dr. McLandsborough and Dr. Kim, for their great support and guidance during my study here.

I would like to give special thanks to Dr. Decker, the department head, and Mr. Peter Salmon, the CFS CPP of IFN Consulting, who generously gave great help, financial support, and gracious encouragement to me.

Many thanks to all of my lab members, past and present: Dr. Yoo Kim, Dr. Xiao Xiao, Dr. Peiyi Shen, Mr. Daniel Colmenares, Dr. Ou Wang, Dr. Phoebe Chen, Dr. Jason Yang, Dr. Yiren Yue, Dr. Weipeng Qi, Dr. Renalison Farias Pereira, Ms. Jinning Liu, Ms. Jiaying Wang, and Mr. Yuejia Xu for their friendship and help during the study period. I would like to thank all of my friends in Amherst, especially Siyue Gao, Xinyi Du, Ruojie Zhang, Hualu Zhou, Yuxi Wang, Tianxi Yang, Shuqi, Yanqi Qu, Weicang Wang, Zipei Zhang, Zili Gao, for their help and friendship.

I want to thank my loving parents and my beautiful sister for their unwavering support and encouragement. Finally, I graciously thank my husband, Quancai Sun for giving me a warm home feeling wherever I am. 


\author{
ABSTRACT \\ THE BIOACTIVE EFFECTS OF CHICORIC ACID AS \\ A FUNCTIONAL FOOD INGREDIENT
}

MAY 2019

YE PENG

B.S., NORTHWEST A \& F UNIVERSITY, CHINA

M.S., UNIVERSITY OF GEORGIA, ATHENS, GEORGIA

Ph.D., UNIVERSITY OF MASSACHUSETTS, AMHERST, MA

Directed by: Professor Yeonhwa Park

Chicoric acid, a hydroxycinnamic acid, has been reported to possess a variety of health benefits, including antivirus, anti-oxidation, anti-inflammation, obesity prevention and neuroprotection effects. However, its role on aging and glucose homeostasis are largely unknown. Therefore, the bioactive effects of chicoric acid on aging and glucose homeostasis were determined using Caenorhabditis elegans (C. elegans) and $\mathrm{C} 2 \mathrm{C} 12$ myotubes, respectively. Our study showed that chicoric acid (25 and $50 \mu \mathrm{M})$ significantly extended the lifespan of $C$. elegans and increased median survival rates. The declines of pumping rate and locomotive activity, two indicators of aging, were delayed by chicoric acid. Chicoric acid also enhanced resistance to oxidative stress compared to the control. These effects were in part via AAK-2 (a homolog of AMP-activated protein kinase) and 
SKN-1 (a homolog of nuclear factor erythroid 2-related factor 2). Furthermore, chicoric acid significantly enhanced glucose uptake and the phosphorylation of protein kinase B (Akt) as an indicator of insulin signaling pathway independent of insulin in C2C12 myotubes. These effects were dependent of the phosphorylation of 5'-AMP-activated protein kinase $\alpha(\mathrm{AMPK} \alpha)$, via increasing the $\mathrm{AMP} / \mathrm{ATP}$ ratio in $\mathrm{C} 2 \mathrm{C} 12$ myotubes. Our study may suggest the potential of chicoric acid to be used as an anti-aging and antidiabetic bioactive compound. 


\section{TABLE OF CONTENTS}

Page

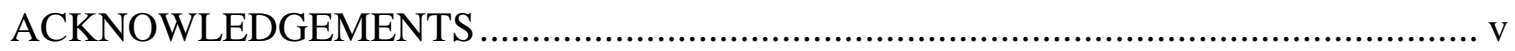

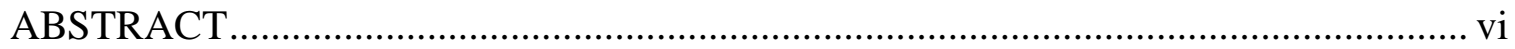

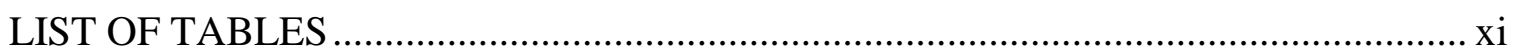

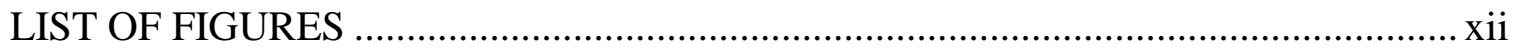

\section{CHAPTER}

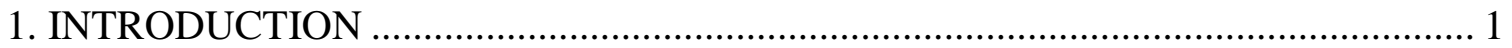

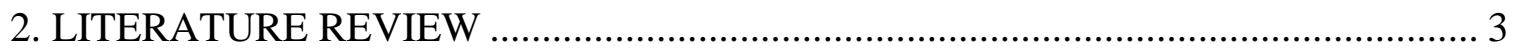

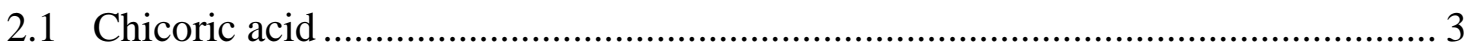

2.2 The biological effects of chicoric acid ....................................................... 4

2.2.1 Anti-viral effects of chicoric acid ........................................................ 4

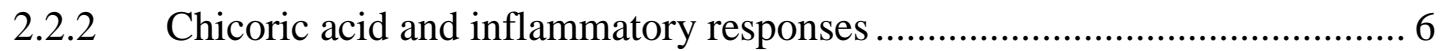

2.2.3 Chicoric acid and glucose metabolism .............................................. 7

2.2.4 Chicoric acid and lipid metabolism \& liver function............................... 8

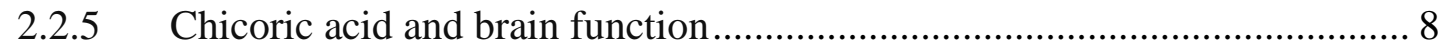

2.2.6 Anti-oxidative stress effects of chicoric acid .......................................... 9

2.2.7 Other bioactivates of chicoric acid …................................................ 10

2.3 Suggested molecular targets of chicoric acid............................................ 20

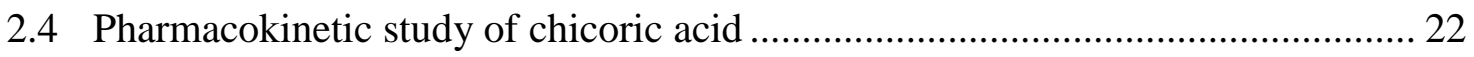


2.5 Conclusion

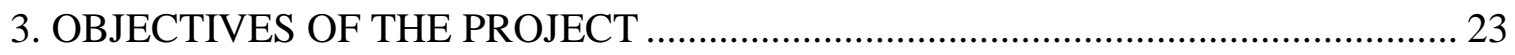

4. EFFECTS OF CHICORIC ACID ON AGING AND GLUCOSE UPTAKE .............. 24

4.1 Effect of chicoric acid on aging in C. elegans and the underlying mechanisms .. 24

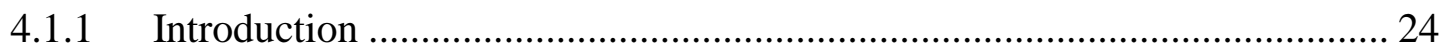

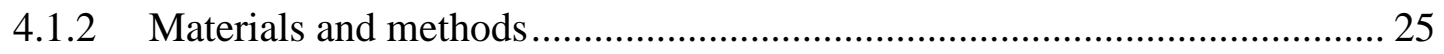

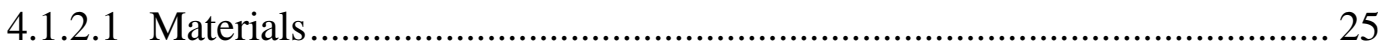

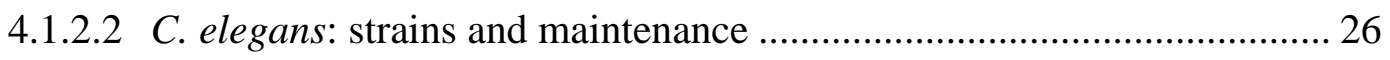

4.1.2.3 Growth rate, body size, and moving speed determination .................... 27

4.1.2.4 Progeny production and pumping rate determination ......................... 27

4.1.2.5 Measurement of intracellular ROS and oxidative stress resistance ....... 28

4.1.2.6 Quantitative real-time PCR ....................................................... 28

4.1.2.7 Intracellular localization of SKN-1::GFP ...................................... 29

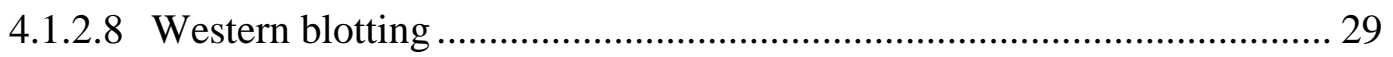

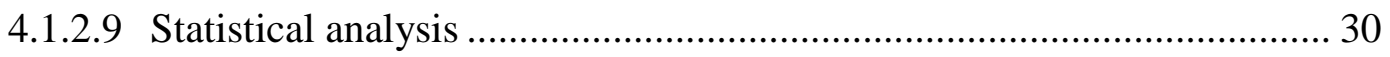

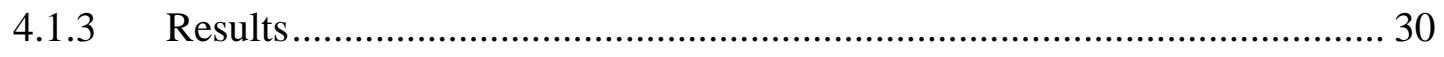

4.1.3.1 Chicoric acid extended the lifespan of $C$. elegans .............................. 31

4.1.3.2 Effect of chicoric acid on the age-associated decline of phenotypes and

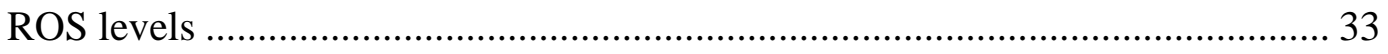

4.1.3.3 Extended lifespan by chicoric acid required AAK-2 and SKN-1 .......... 35 
4.1.3.4 Chicoric acid might extend $C$. elegans lifespan through upregulation of $a a k-2$ and $s k n-1$ 38

4.1.3.5 Chicoric acid enhanced oxidative stress resistance in wild type N2 worms but not aak-2 mutants 41

4.1.4 Discussion 42

4.2 Effect of chicoric acid on glucose uptake and the underlying mechanisms 47

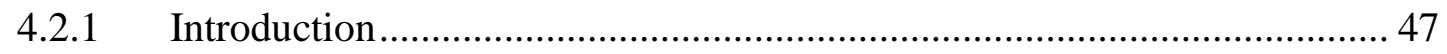

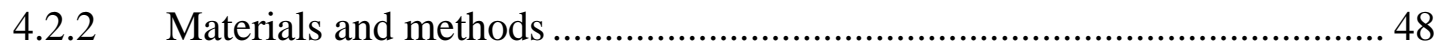

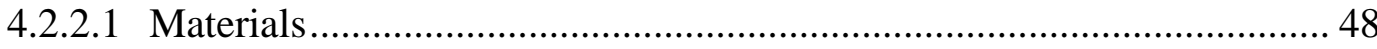

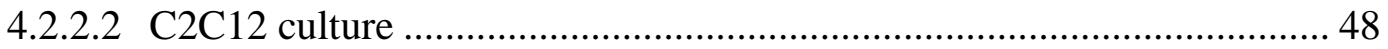

4.2.2.3 Glucose uptake assay ................................................................. 49

4.2.2.4 Cell membrane protein extraction ................................................ 50

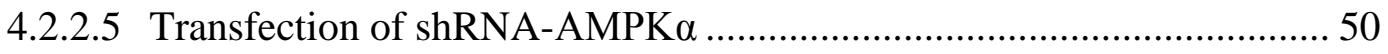

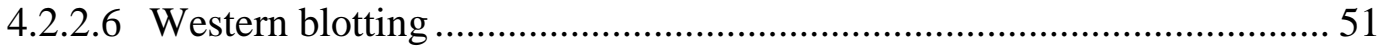

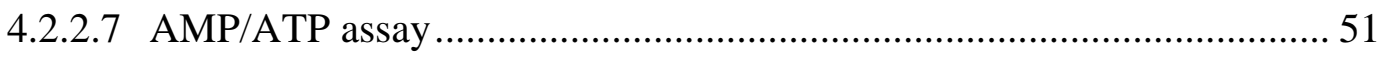

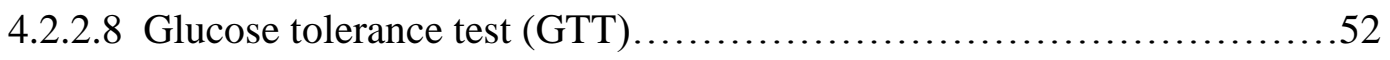

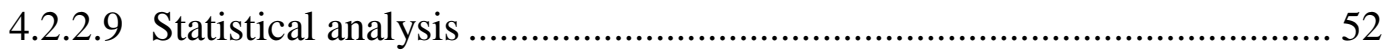

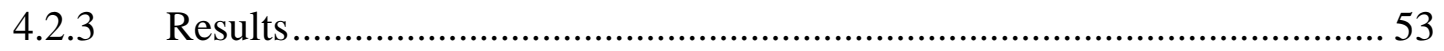

4.2.3.1 Chicoric acid promoted glucose uptake .......................................... 53

4.2.3.2 Effects of chicoric acid on Akt activation and GLUT4 translocation in

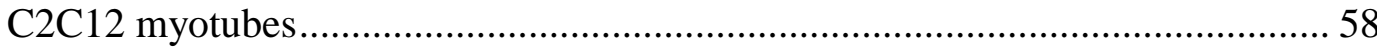


4.2.3.3 Effects of chicoric acid on the AMPK $\alpha$ signaling pathway in $\mathrm{C} 2 \mathrm{C} 12$ myotubes .

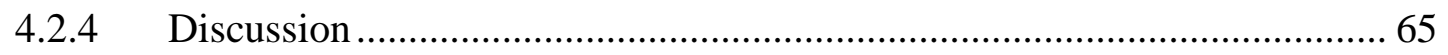

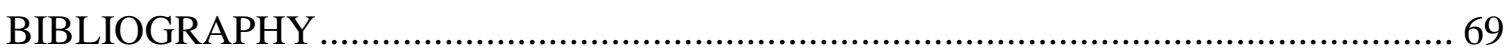




\section{LIST OF TABLES}

Table $\quad$ Page

2.1 In vitro effects of chicoric acid in the treatment of various disorders..................11

2.2 In vivo effects of chicoric acid in the treatment of various disorders...............16

4.1 Effect of chicoric acid on median lifespan of wild type and mutants................37 


\section{LIST OF FIGURES}

Figure

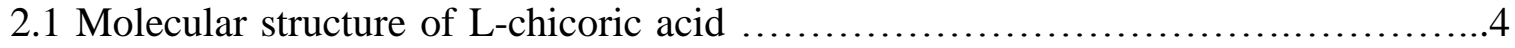

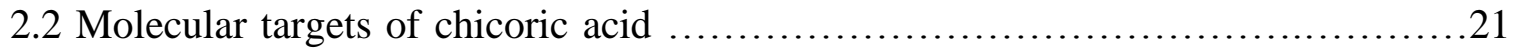

4.1 Survival of wild-type $C$. elegans treated with chicoric acid ......................... 31

4.2 Effect of chicoric acid on growth rate, worm size and progeny production

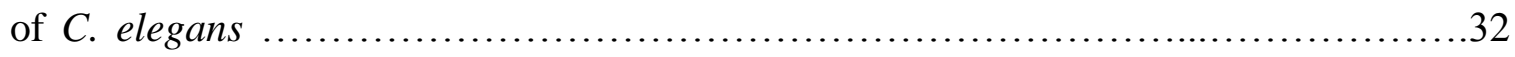

4.3 Chicoric acid ameliorated the age-related decline of movement and pumping rate.

4.4 Effect of chicoric acid on the oxidative stress sensitivity of wild type N2

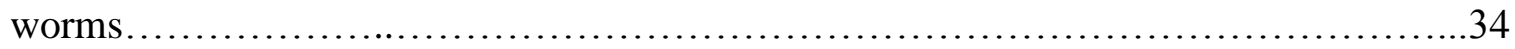

4.5 Survival of several mutant strains treated with chicoric acid.......................38

4.6 Chicoric acid might extend $C$. elegans lifespan via the regulation of AAK-2 and

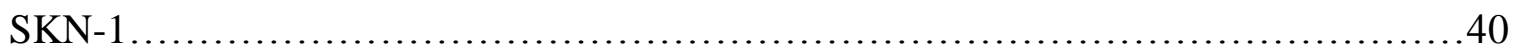

4.7 Chicoric acid enhanced the stress resistance of wild type worms but not aak-2

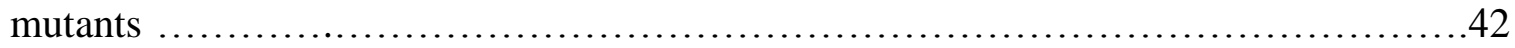

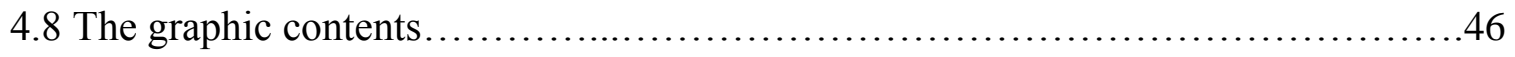

4.9 Chicoric acid promoted glucose uptake in $\mathrm{C} 2 \mathrm{C} 12$ myotube.......................55

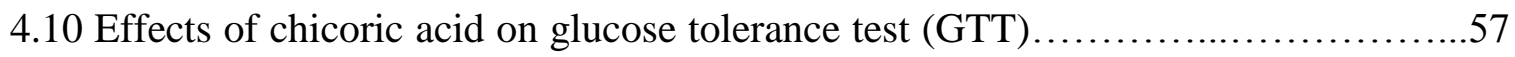

4.11 Activation of Akt and translocation of glucose transporter 4 (GLUT4) by chicoric

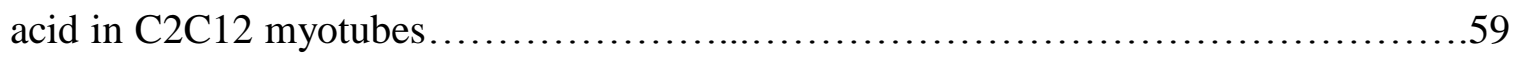

4.12 Activation of AMP-activated protein kinase $\alpha(\mathrm{AMPK} \alpha)$ pathway by chicoric acid in

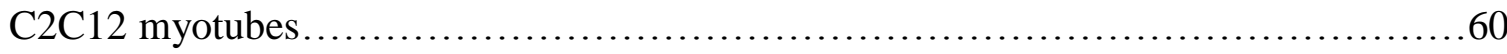


4.13 Increased glucose uptake and activation of Akt by chicoric acid were abolished by

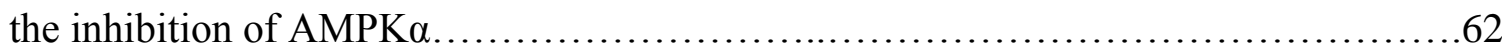

4.14 Increased glucose uptake and activation of Akt by chicoric acid were abolished by

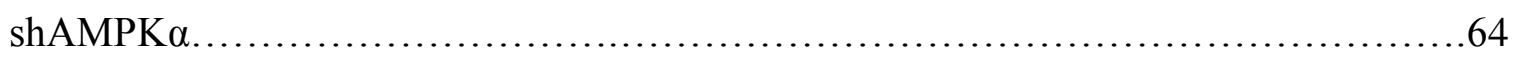

4.15 Chicoric acid enhanced AMP/ATP ratio in $\mathrm{C} 2 \mathrm{C} 12$ myotubes...................65

4.16 The graphic contents................................................. 68 


\section{CHAPTER 1}

\section{INTRODUCTION}

Chicoric acid is a hydroxycinnamic acid presents in the roots of a large number of plants (63 genera and species), including many plants grown in the Mediterranean area (1), and many of these plants have been consumed traditionally as food ingredients and alternative medicines for some time (2,3). Because chicoric acid is found in especially high amounts in chicory (Cichorium intybus), purple coneflower (Echinacea purpurea), and basil, the acid is often used as a marker for the quality check of herbal products from these plants (4). More recently, an increasing number of publications have reported the beneficial effects of chicoric acid in cell culture and animal studies. However, its role on aging and glucose homeostasis are largely unknown.

Aging is defined as a progressive decline of intrinsic physiological functions, resulting in an increased risk of death (5). Considering that aging is one of the key risk factors associated with many chronic diseases, slowing aging is of great importance to prevent age-associated diseases and improve the health span of life (6). A previous study reported that chicoric acid extend lifespan in Caenorhabditis elegans (7), its effects on aging-related phenotypes and its underlying mechanisms, however, remain to be determined.

Type 2 diabetes mellitus (T2DM) has been recognized as a major public health challenge throughout the world in recent decades $(8,9)$. Impaired glucose uptake in muscle tissues is the primary associated with hyperglycemia in T2DM (10). Previous in vitro studies reported that chicoric acid ameliorated insulin resistance in human hepatoma cells (11). However, the effects of chicoric acid on muscle cells and its underlying 
mechanism are still unclear. Here, $\mathrm{C} 2 \mathrm{C} 12$ myotubes, derived from murine skeletal muscle cells and a C57BL/6J mice model were used to determine the role of chicoric acid on glucose homeostasis and its underlying mechanisms were determined. 


\section{CHAPTER 2}

\section{LITERATURE REVIEW}

\subsection{Chicoric acid}

Chicoric acid, a hydroxycinnamic acid that is a member of the phenylpropanoid family, contains two caffeoyl units (2). Chicoric acid mainly presents in two forms: first, the most abundant natural form of chicoric acid is L-chicoric acid (Figure 1); second, the acid presents in the stereoisomer meso-chicoric acid (i.e., dicaffeoyl-meso-tartaric acid or di-E-caffeoyl-(2R-3S)-(-)-tartaric acid) (1).

Chicoric acid is present in the roots of a large number of plants (63 genera and species), including many plants grown in the Mediterranean area (1), and many of these plants have been consumed as alternative medicines or food supplements for some time $(2,3)$. Because chicoric acid is found in especially high amounts in chicory (Cichorium intybus), purple coneflower (Echinacea purpurea), and basil, the acid is often used as a marker for the quality check of herbal products from these plants (4). The roots of chicory and purple coneflower are usually baked, grounded and used as a coffee substitute in Europe (12). In Turkey, an herbal tea made from chicory has been used historically for the treatment of diabetes, epilepsy, hemorrhoids, inflammation and digestive disorders (13). The plant roots containing chicoric acid have been used in Asian traditional medicine as a tonic for curing infectious diseases, inflammatory diseases, eye diseases, and nerves injuries $(13,14)$. Commercial products made from purple coneflower are currently popular alternative medicines widely used in North America for cold and flu prevention (15). More recently, an increasing number of publications have reported the beneficial effects of chicoric acid in cell culture and animal studies. This 
review summarizes these health benefit studies and the underlying mechanisms of chicoric acid.

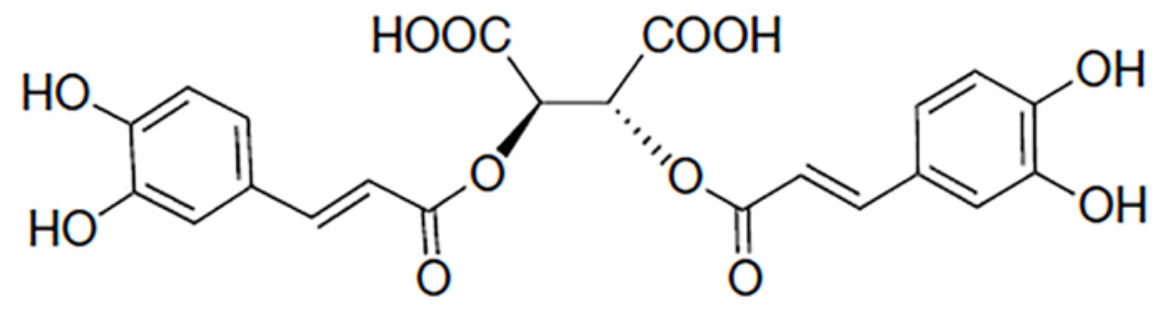

Figure 2.1. Molecular structure of L-chicoric acid. The most abundant natural form of chicoric acid is L-chicoric acid, i.e., (-)-chicoric acid, 2,3-dicaffeoyl-L-tartaric acid, 2,3O-dicaffeoyltartaric acid, $2 R, 3 R$-O-dicaffeoyltartaric acid, or di-E-caffeoyl-(2R-3R)-(-)tartaric acid.

\subsection{The Biological effects of chicoric acid}

The main benefits of chicoric acid include antivirus, anti-inflammation, glucose and lipid homeostasis, neuroprotection, and anti-oxidation effects. Biological activities and related mechanisms of chicoric acid from in vitro and in vivo studies are summarized in Tables 1 and 2.

\subsubsection{Anti-viral effects of chicoric acid}

The first reported bioactive effect of chicoric acid is its ability to inhibit infection with human immunodeficiency virus 1 (HIV-1) (16). Several studies reported that chicoric acid inhibits infection with HIV-1 by deactivating HIV-1 integrase (16-23). HIV-1 integrase is a multidomain enzyme required for the integration of viral DNA into the host genome, a critical step in viral replication (24). The inhibition of HIV-1 integration by chicoric acid results in the stopping virus replication, leading to increased T-lymphoblastoid cell viability $(17-19,21,22)$. It has been further suggested that chicoric 
acid decreases integrase binding site activity, including: the downregulation of HIV 3'end processing products (16), the occupation of HIV-1 integrase catalytic core (20), the chelation of integrase divalent cations (17), the increase of long terminal repeat circle formation (21), and the inhibition of integrase-mediated catalysis (18). Hu et al. (25) reported that the mutation of HIV integrase might result in the blocking of chicoric acid specific binding sites and King et al. (26) further reported that the mutation on glycine to serine at position 140 (G140S) of HIV integrase reduced chicoric acid's effects on HIV infection, suggesting that the integrase G140S might be a target site of chicoric acid. There are a few studies that are using new computational techniques including molecular docking and quantitative structure-activity relationship (QSAR) analysis to identify the binding sites of chicoric acid with HIV-1 integrase $(25,27,28)$. Chicoric acid binds HIV1 integrase at its two arms, including the s-cis/s-cis isomer and s-cis/s-trans isomer arrangements (27). Based on the observation that the s-cis/s-cis isomer exhibits the most stable binding, this site was suggested to be the target of chicoric acid to inhibit HIV integrase. Another study using QSAR analysis indicated that the poly aromatic rings of chicoric acid are central linkers in binding to HIV-1 integrase (28). In addition to integrase, reverse transcriptase is another potential target of chicoric acid $(23,29)$, as chicoric acid downregulates reverse transcriptase of HIV-1 through the inhibition of the trans-activator of transcription, an important protein promoting HIV-1 reverse transcription (29).

Although chicoric acid may be a potential treatment for HIV, there are several limitations of using naturally occurring chicoric acid as a treatment, such as poor stability and limited cell permeability due to diacid moiety (30). To overcome these limitations, 
chicoric acid analogs have been introduced, such as an analog of a decarboxyl compound, while extending a caffeoyl group on 3,4,5-trihydroxycinnamoyl sidechains, which showed improved stability and cell bioavailability (30-32). This analog also exhibited the high potency against HIV integrase infectivity (30-32).

\subsubsection{Chicoric acid and inflammatory responses}

Chicoric acid has been found to ameliorate inflammation induced by lipopolysaccharides (LPS) in both cell culture and mice. Reduced inflammation was associated with down-regulations of nuclear factor $\kappa \mathrm{B}(\mathrm{NF}-\kappa \mathrm{B})$ and tumor necrosis factor $\alpha($ TNF- $\alpha)$ (33-36), which are two major regulators of inflammation responses (37-39). Several other pro-inflammatory factors - including nitric oxide synthase (iNOS), cyclooxygenase-2 (COX-2), prostaglandin $\mathrm{E}_{2}\left(\mathrm{PGE}_{2}\right)$, interleukin-1 $\beta$ (IL-1 $\left.\beta\right)$, IL-12 and IL-18 have been also reported to be downregulated by chicoric acid (33, 35, 40-43). However, two relevant studies showed results inconsistent with the above. Matthias et al. (44) reported that LPS inhibited NF- $\kappa \mathrm{B}$ expression, which was reversed by chicoric acid treatments in Jurkat E6.1 leukaemic T-cell lymphoblasts. The other reported that echinacea extracts (containing chicoric acid) upregulated LPS-induced TNF- $\alpha$ in rat alveolar macrophages (45). Inconsistencies may be due to dosage and types of LPS used (i.e., 0.1 vs. $1 \mu \mathrm{g} / \mathrm{ml})(34,43,46-48)$ and/or other components present in treatments $(36$, 49). Overall, since the pro-inflammatory factors above are related to the occurrence of many chronic diseases, most research suggests chicoric acid may be considered a preventive tool for inflammation-associated diseases $(33,35,40-43)$, however, further evaluation is needed. 


\subsubsection{Chicoric acid and glucose metabolism}

Chicoric acid has been reported to promote glucose uptake in muscle cells and hepatocytes, through activating the insulin receptor substrate/ phosphoinositide 3-kinase /protein kinase B (Akt) pathway $(11,50,51)$. Zhu et al.(51,52) suggested that chicoric acid activated Akt via the AMP-activated protein kinase $\alpha$ (AMPK $\alpha$ )-dependent mechanism, which is a master regulator for energy homeostasis and also plays a key role in glucose metabolism. However, the mechanism underlying how chicoric acid induces AMPK $\alpha$ activation is not known currently. In addition to AMPK $\alpha$, glucosidase, a digestive enzyme for carbohydrates, was reported to be suppressed by chicory (53), which can contribute to reduced glucose levels $(54,55)$. Another enzyme, protein tyrosine phosphatase 1B (PTP1B) negatively regulates the insulin signaling pathway by inhibiting the activity of insulin receptor kinase (56). Two studies with molecular docking showed the molecular interactions between the allosteric site of PTP1B and chicoric acid, which suggests chicoric acid might inhibit PTP1B and further activate the insulin signaling pathway $(57,58)$.

In in vivo studies, chicoric acid (or leafy extracts of echinacea or basil) reduced streptozotocin induced-hyperglycemia in mice $(50,52,59,60)$. In these studies, the protection of pancreatic $\beta$-cells by chicoric acid was attributed to its regulation of apoptosis related-genes, including c-Jun N-terminal kinase (JNK), B-cell lymphoma 2associated X/B-cell lymphoma 2 (Bax/Bcl-2) ratio, and pancreatic duodenal homeobox 1 (PDX-1) (61). In addition, as stated in the previous section, chicoric acid downregulates several pro-inflammatory cytokines and mediators and these metabolic inflammatory 
cytokines are related to the impairment of glucose homeostasis (62), indicating that chicoric acid might improve glucose homeostasis via regulating inflammatory responses, including those associated with regulators $\mathrm{COX}-2$, mitogen-activated protein kinase (MAPK), cAMP response element binding protein (CREB) and NF- $\kappa \mathrm{B}(51,52)$,

\subsubsection{Chicoric acid and lipid metabolism \& liver function}

Chicoric acid has been found to reduce high-fat-diet-induced weight gain in mice $(63,64)$. This was in part via the inhibition of peroxisome proliferator-activated receptor$\gamma(\operatorname{PPAR} \gamma)$ and CCAAT/enhancer binding protein $\alpha(\mathrm{C} / \mathrm{EBP} \alpha)$, two critical transcription factors in adipocyte differentiation and lipid accumulation, while increasing the secretion of adiponectin (64). Similarly, a few studies suggested that chicoric acid protected the liver from high-fat- or alcohol-induced fat accumulation and hepatic steatosis (63-65). The hepatoprotective effects of chicoric acid were suggested to be due in part to a decrease in the Bax/Bcl-2 ratio and the inhibition of fatty acid synthase (FAS) and proinflammatory cytokines, including TNF- $\alpha$, IL-6, COX-2 and JNK in the liver $(63,64)$.

\subsubsection{Chicoric acid and brain function}

There is a report that chicoric acid treatment ameliorated restraint stress-induced behavioral despair and depression in mice (66). This neuro-protective effects of chicoric acid may result from its upregulation of neurotransmitters, including noradrenaline, dopamine, and 5-hydroxy tryptamine in the whole brain region of mice (66). Since restraint stress is an important factor contributing to disorders and behavioral changes (such as depression, memory loss, anxiety, and learning disability), the possible 
alleviation of restraint stress by chicoric acid suggests the potential application of chicoric acid for the above-mentioned brain disorders (66). Others reported that chicoric acid protected neurons from memory impairment, amyloidogenesis, and hippocampus shrinkage induced by LPS and D-galactose in mice $(33,34,67)$. They also found that chicoric acid inhibited the expression of amyloid $\beta$ and its downstream enzyme, neuronal $\beta$-secretase 1 , both of which are known factors contributing to the disruption of neural connectivity and neuronal death (68). Meanwhile, chicoric acid upregulated brain-derived neurotrophic factor, which is a canonical nerve growth factor supporting the survival of existing neurons and promoting the growth of new neurons and synapses (69). Although limited, these findings suggest the potential protective activity of chicoric acid on controlling the pathogenesis of neuro-degenerative diseases.

\subsubsection{Anti-oxidative stress effects of chicoric acid}

Oxidative stress is defined as the imbalance between the generation of reactive oxygen species (ROS) and a physical ability of detoxification or damage restoration associated with ROS (70). ROS could act as cellular massagers but cause physical damages through disruptions of normal cell signaling pathways (71). Chicoric acid has been found to have a high oxygen radical scavenging capacity, reducing the ROS level and protecting cells from free radical-induced cytotoxicity $(34,53,72-75)$. Moreover, chicoric acid increased the generation of anti-oxidative enzymes that contribute to the reduction of ROS levels, i.e., glutathione, glutathione peroxidase, superoxide dismutase, chloramphenicol acetyl transferase, heme oxygenase and $\mathrm{NAD}(\mathrm{P}) \mathrm{H}$ dehydrogenase in various cells $(15,21,25,43,49,61,62,71,76,77)$. The underlying mechanism of the 
antioxidative effects of chicoric acid is attributed to the enhanced nuclear translocation of nuclear factor erythroid 2-related factor 2 (Nrf-2) and the level of peroxisome proliferator-activated receptor- $\gamma$ coactivator $\alpha(\mathrm{PGC}-1 \alpha)(34,73,75)$. The activation of Nrf-2 and PGC-1 $\alpha$ inhibit ROS-induced cytotoxicity by upregulation of antioxidant response-related genes and promotion of the mitochondrial antioxidant defense system, respectively $(78,79)$. Since oxidative stress is closely related to the development of certain cancers and chronic diseases, all these findings suggest the potential future application of chicoric acid for oxidative stress-associated diseases.

\subsubsection{Other bioactivities of chicoric acid}

Chicoric acid at relatively higher concentrations $(105-315 \mu \mathrm{M})$ has been reported to inhibit cancer cell growth via inhibiting cell proliferation, promoting cell apoptosis, and deactivating telomerase through upregulation of DNA fragmentation, cleaved caspase-9, and cleaved poly (ADP-ribose) polymerase (80).

Another study suggested that chicoric acid ameliorated ultraviolet A irradiationinduced dermal fibroblast senescence by the inhibition of matrix metalloproteinase-3 activity (81). As dermal fibroblast senescence is a hallmark of intrinsic and ultravioletmediated aging (77), this indicates the potential role of chicoric acid in aging. 
Table 2.1 In vitro effects of chicoric acid in the treatment of various disorders.

\begin{tabular}{|c|c|c|c|c|c|c|}
\hline Disorders & Models & $\begin{array}{l}\text { Dose } \\
(\mu \mathrm{M})\end{array}$ & $\begin{array}{l}\text { Duration } \\
\text { (hrs) }\end{array}$ & Effects & Suggested mechanisms & References \\
\hline \multirow[t]{8}{*}{$\begin{array}{l}\text { Anti-viral } \\
\text { effects }\end{array}$} & $\begin{array}{l}\text { H9 \& MT-2 human T- } \\
\text { lymphoblastoid }\end{array}$ & $\begin{array}{l}1.05- \\
10.5\end{array}$ & 48 & $\begin{array}{l}\uparrow \text { infected cell viability; } \\
\downarrow \text { HIV-1 integrase } \\
\text { activity }\end{array}$ & $\begin{array}{l}\downarrow \text { HIV } 3 \text { '-end processing } \\
\text { products }\end{array}$ & $(16)$ \\
\hline & $\begin{array}{l}\text { H9 \& MT-2 human T- } \\
\text { lymphoblastoid }\end{array}$ & 0.4 & 48 & $\begin{array}{l}\downarrow \text { HIV-1 integrase } \\
\text { activity }\end{array}$ & $\begin{array}{l}\downarrow \text { HIV integrase catalytic } \\
\text { core }\end{array}$ & $(20)$ \\
\hline & $\begin{array}{l}\text { MT-2 human T- } \\
\text { lymphoblastoid }\end{array}$ & 105 & 48 & $\begin{array}{l}\downarrow \text { HIV-1 reverse } \\
\text { transcriptase activity }\end{array}$ & $\begin{array}{l}\downarrow \text { HIV-1 envelope } \\
\text { glycoprotein } 120\end{array}$ & (29) \\
\hline & $\begin{array}{l}\text { MT-2 human T- } \\
\text { lymphoblastoid }\end{array}$ & 0.4 & 72 & $\begin{array}{l}\uparrow \text { infected cell viability; } \\
\downarrow \text { HIV-1 reverse } \\
\text { transcriptase activity; } \downarrow \\
\text { HIV-1 reverse } \\
\text { transcriptase release }\end{array}$ & N/A & $(23)$ \\
\hline & $\begin{array}{l}\text { MT-2 human T- } \\
\text { lymphoblastoid }\end{array}$ & 25 & 72 & $\begin{array}{l}\downarrow \text { HIV-1 integrase } \\
\text { replication }\end{array}$ & N/A & (19) \\
\hline & $\begin{array}{l}\text { MAGI human cervical } \\
\text { epithelial carcinoma }\end{array}$ & 100 & 52 & $\begin{array}{l}\downarrow \text { HIV-1 integrase } \\
\text { activity; }\end{array}$ & $\begin{array}{l}\downarrow \text { HIV-1 Tat protein } \\
\text { synthesis }\end{array}$ & $(82)$ \\
\hline & $\begin{array}{l}\text { H9 \& MT-2 human T- } \\
\text { lymphoblastoid }\end{array}$ & $\begin{array}{l}0.1- \\
10\end{array}$ & 24 & $\begin{array}{l}\downarrow \text { viral replication; } \\
\downarrow \text { HIV-1 integrase } \\
\text { activity }\end{array}$ & $\begin{array}{l}\uparrow \text { integrase divalent } \\
\text { cations chelation }\end{array}$ & $(17)$ \\
\hline & MT-4 human T- & 70 & 2 & $\downarrow$ viral replication cycle; & $\downarrow$ HIV-1 envelope & $(83)$ \\
\hline
\end{tabular}




\begin{tabular}{|c|c|c|c|c|c|c|}
\hline & lymphoblastoid & & & $\downarrow$ viral entry & glycoprotein 120 & \\
\hline & $\begin{array}{l}\text { MT-2 human T- } \\
\text { lymphoblastoid }\end{array}$ & 4.2 & 72 & $\begin{array}{l}\uparrow \text { infected cell viability; } \\
\downarrow \text { HIV-1 integrase } \\
\text { activity }\end{array}$ & $\begin{array}{l}\uparrow \text { long terminal repeat } \\
\text { circle formation }\end{array}$ & $(21)$ \\
\hline & $\begin{array}{l}\text { H9 \& MT-2 human T- } \\
\text { lymphoblastoid }\end{array}$ & 50 & $1-4$ & $\begin{array}{l}\downarrow \text { HIV-1 integrase } \\
\text { activity }\end{array}$ & $\begin{array}{l}\downarrow \text { integrase binding site } \\
\text { activity }\end{array}$ & $(22)$ \\
\hline & $\begin{array}{l}\text { H9 \& MT-2 human T- } \\
\text { lymphoblastoid }\end{array}$ & 25 & 1 & $\begin{array}{l}\downarrow \text { HIV-1 integrase } \\
\text { activity }\end{array}$ & $\begin{array}{l}\downarrow \text { integrase-mediated } \\
\text { catalysis }\end{array}$ & (18) \\
\hline \multirow[t]{4}{*}{$\begin{array}{l}\text { Glucose } \\
\text { metabolism }\end{array}$} & $\begin{array}{l}\text { L6 rat myotubes \& } \\
\text { insulinoma-derived ISN- } \\
1 \text { pancreatic } \beta \text { cells }\end{array}$ & $\begin{array}{l}200 \\
\& \\
20- \\
100\end{array}$ & $1-2$ & $\begin{array}{l}\uparrow \text { glucose uptake; } \\
\uparrow \text { insulin secretion }\end{array}$ & N/A & (11) \\
\hline & $\begin{array}{l}\text { INS-1 pancreatic } \beta \text { - } \\
\text { cells; L6 myotubes; } \\
\text { isolated hepatocytes } \\
\text { (rat) }\end{array}$ & $\begin{array}{l}105- \\
210\end{array}$ & $1-3$ & $\begin{array}{l}\uparrow \text { glucose uptake; } \\
\uparrow \text { insulin secretion }\end{array}$ & N/A & $(50)$ \\
\hline & $\begin{array}{l}\text { HepG2 human } \\
\text { hepatoma }\end{array}$ & 100 & 24 & $\begin{array}{l}\downarrow \text { insulin resistance; } \\
\downarrow \text { NO \& ROS }\end{array}$ & $\begin{array}{l}\uparrow \text { GLUT2 translocation; } \\
\uparrow \mathrm{Akt} ; \downarrow \text { MAPK; } \downarrow \text { NF- } \\
\kappa \mathrm{B}\end{array}$ & $(51)$ \\
\hline & $\begin{array}{l}\text { HepG2 human } \\
\text { hepatoma }\end{array}$ & 100 & 24 & $\uparrow$ glucose uptake & $\begin{array}{l}\uparrow \text { IRS-1; } \uparrow \text { Akt; } \uparrow \\
\text { AMPK } \alpha ; \uparrow \text { SIRT1; } 1 \\
\text { GSK-3 } ; \downarrow \downarrow \text { CREB }\end{array}$ & $(52)$ \\
\hline \multirow[t]{3}{*}{$\begin{array}{l}\text { Inflammation } \\
\text { responses }\end{array}$} & $\begin{array}{l}\text { RAW } 264.7 \text { mouse } \\
\text { macrophage }\end{array}$ & 4.2 & 4 & N/A & $\downarrow N F-\kappa B ; \downarrow$ TNF- $\alpha$ & $(36)$ \\
\hline & Macrophage & N/A & N/A & $\downarrow$ cell inflammation & $\downarrow$ TNF- $\alpha$ & $(35)$ \\
\hline & Jurkat E6.1 human & 17 & 4 & N/A & $\uparrow \mathrm{NF \kappa B}$ & $(44)$ \\
\hline
\end{tabular}




\begin{tabular}{|c|c|c|c|c|c|c|}
\hline & $\begin{array}{l}\text { leukemic T-cell } \\
\text { lymphoblast }\end{array}$ & & & & & \\
\hline & $\begin{array}{l}\text { Mouse peripheral blood } \\
\text { mononuclear cells }\end{array}$ & $\begin{array}{l}0.5- \\
4.2\end{array}$ & 4 & $\uparrow$ immune homeostasis & $\uparrow$ IL-2; $\uparrow$ IFN- $\gamma ; \downarrow$ IL-4 & $(41)$ \\
\hline & BV-2 mouse microglia & 80 & 4 & $\downarrow$ cell inflammation & $\begin{array}{l}\downarrow \text { iNOS } ; \downarrow \text { COX-2; } \downarrow \\
\text { PGE } 2 ; \downarrow \text { IL- } 1 \beta ; \downarrow \text { TNF- } \alpha\end{array}$ & (33) \\
\hline & $\begin{array}{l}\text { Human umbilical vein } \\
\text { endothelial cells }\end{array}$ & $\begin{array}{l}12.5- \\
100\end{array}$ & 24 & $\begin{array}{l}\downarrow \text { endothelial } \\
\text { dysfunction; } \downarrow \text { cell } \\
\text { apoptosis; } \uparrow \text { cell } \\
\text { viability; } \downarrow \text { ROS }\end{array}$ & $\begin{array}{l}\uparrow \mathrm{SOD} ; \uparrow \text { eNOS; } \downarrow \\
\text { Bax/Bcl-2; } \downarrow \text { cleaved } \\
\text { caspase-3; } \downarrow \text { MAPK; } \downarrow \\
\text { NF- } \mathrm{B}\end{array}$ & $(42)$ \\
\hline & $\begin{array}{l}\text { HT-29 human colorectal } \\
\text { adenocarcinoma }\end{array}$ & 42 & 12 & $\downarrow$ cell inflammation & $\begin{array}{l}\downarrow \mathrm{NF}-\kappa \mathrm{B} ; \downarrow \text { COX-2; } \downarrow \\
\mathrm{IL}-1 \beta ; \downarrow \text { IL-18 }\end{array}$ & $(43)$ \\
\hline \multirow[t]{8}{*}{$\begin{array}{l}\text { Oxidative } \\
\text { stress }\end{array}$} & Human plasma & 1 & 5 & $\begin{array}{l}\downarrow \mathrm{Cu} \text { (II)-catalyzed LDL } \\
\text { oxidation }\end{array}$ & N/A & $(84)$ \\
\hline & $\begin{array}{l}\text { RAW264.7 mouse } \\
\text { macrophage }\end{array}$ & $\begin{array}{l}16- \\
32\end{array}$ & 20 & $\downarrow$ oxidative stress & $\begin{array}{l}\downarrow \text { PGE2; } \downarrow \text { TNF- } \alpha ; \downarrow \text { IL- } \\
1 \beta ; \downarrow \text { NF- } \kappa B ; \downarrow \text { Akt }\end{array}$ & $(47)$ \\
\hline & $\begin{array}{l}\text { RAW264.7 mouse } \\
\text { macrophage }\end{array}$ & $\mathrm{N} / \mathrm{A}$ & 20 & $\begin{array}{l}\downarrow \text { oxidative stress \& } \downarrow \\
\text { NO }\end{array}$ & $\begin{array}{l}\uparrow \mathrm{GSH} ; \downarrow \text { iNOS; } \downarrow \text { NF- } \\
\kappa \mathrm{B}\end{array}$ & $(48)$ \\
\hline & L6 rat myotubes & $5-50$ & 1 & $\downarrow$ ROS & $\begin{array}{l}\uparrow \mathrm{GPx} ; \uparrow \mathrm{SOD} ; \uparrow \mathrm{p}- \\
\mathrm{AMPK} \alpha ; \uparrow \mathrm{PGC}-1 \alpha\end{array}$ & $(73)$ \\
\hline & $\begin{array}{l}\text { RGC-5 rat retinal } \\
\text { ganglion cells }\end{array}$ & 0.025 & 24 & $\begin{array}{l}\uparrow \text { cell viability \& } \\
\downarrow \text { ROS }\end{array}$ & $\begin{array}{l}\downarrow \text { cleaved PARP; } \downarrow \\
\text { cleaved caspase-3 }\end{array}$ & (67) \\
\hline & $\begin{array}{l}\text { RAW264.7 mouse } \\
\text { macrophage }\end{array}$ & 340 & 19 & $\downarrow$ oxidative stress & $\begin{array}{l}\downarrow \text { MyD88; } \downarrow \text { iNOS; } \downarrow \\
\text { TNF- } \alpha\end{array}$ & $(65)$ \\
\hline & $\begin{array}{l}\text { HepG2 human } \\
\text { hepatoma }\end{array}$ & 100 & 24 & $\downarrow \mathrm{NO} ; \downarrow$ ROS & $\begin{array}{l}\downarrow \mathrm{COX}-2 ; \downarrow \text { iNOS; } \downarrow \text { NF- } \\
\kappa \mathrm{B}\end{array}$ & $(51)$ \\
\hline & $\begin{array}{l}\text { Daudi \& Namalwa B } \\
\text { lymphocyte; JeKo-1 }\end{array}$ & $\begin{array}{l}21- \\
105\end{array}$ & $12-48$ & $\begin{array}{l}\downarrow \text { B cell activating factor } \\
\text { belonging to the TNF }\end{array}$ & $\downarrow \mathrm{NF}-\kappa \mathrm{B} ; \downarrow$ I $\kappa \mathrm{B}$ & $(85)$ \\
\hline
\end{tabular}




\begin{tabular}{|c|c|c|c|c|c|c|}
\hline & $\begin{array}{l}\text { mantle cell lymphoma, } \\
\text { THP-1 monocytes \& } \\
\text { HepG2 hepatoma } \\
\text { (human) }\end{array}$ & & & family (BAFF) & & \\
\hline & BV-2 mouse microglia & 80 & 4 & $\downarrow$ oxidative stress & $\begin{array}{l}\downarrow \text { NF- } \kappa \text { B; } \downarrow \text { MAPK; } \uparrow \\
\text { Nrf2 }\end{array}$ & (34) \\
\hline & $\begin{array}{l}\text { SH-SY5Y human } \\
\text { neuroblastoma }\end{array}$ & 50 & 24 & $\begin{array}{l}\downarrow \text { oxidative stress; } \\
\uparrow \text { cell viability }\end{array}$ & $\begin{array}{l}\uparrow \text { Nrf2; } \uparrow \text { HO-1; } \uparrow \text { NQO- } \\
1 ; \uparrow \text { CAT; } \uparrow \text { GSH; } \downarrow \\
\text { TNF- } \alpha ; \downarrow \text { IL-1 } \beta ; \downarrow \\
\text { malondialdehyde }\end{array}$ & $(75)$ \\
\hline \multirow[t]{6}{*}{ Others } & $\begin{array}{l}\text { HL- } 7702 \text { human } \\
\text { hepatocytes \& HepG } 2 \\
\text { human hepatoma }\end{array}$ & $\begin{array}{l}20- \\
200\end{array}$ & 48 & $\begin{array}{l}\uparrow \text { infected cell viability; } \\
\downarrow \text { HBV activity }\end{array}$ & $\begin{array}{l}\downarrow \text { HBV surface \& } \\
\text { envelope antigen }\end{array}$ & $(86)$ \\
\hline & $\begin{array}{l}\text { Caco-2 \& HCT-116 } \\
\text { epithelial colorectal } \\
\text { adenocarcinoma } \\
\text { (human) }\end{array}$ & $\begin{array}{l}105- \\
315\end{array}$ & $24-48$ & $\begin{array}{l}\downarrow \text { cell proliferation; } \\
\uparrow \text { cell apoptosis; } \\
\downarrow \text { telomerase activity }\end{array}$ & $\begin{array}{l}\uparrow \text { DNA fragmentation; } \uparrow \\
\text { cleaved caspase- } 9 ; \uparrow \\
\text { cleaved PARP }\end{array}$ & $(80)$ \\
\hline & $\begin{array}{l}\text { HeLa cervical } \\
\text { carcinoma \& MCF-7 } \\
\text { breast carcinoma } \\
\text { (human) }\end{array}$ & 0.05 & 24 & $\begin{array}{l}\downarrow \text { doxorubicin-induced } \\
\text { cell death }\end{array}$ & N/A & $(87)$ \\
\hline & Human skin fibroblasts & 2 & 24 & $\begin{array}{l}\downarrow \text { dermal fibroblasts } \\
\text { senescence }\end{array}$ & $\downarrow$ MMP-3 & $(81)$ \\
\hline & $\begin{array}{l}\text { 3T3-L1 mouse } \\
\text { preadipocytes }\end{array}$ & 100 & 48 & $\begin{array}{l}\uparrow \text { cell apoptosis } \& \downarrow \\
\text { mitochondrial membrane } \\
\text { potential }\end{array}$ & $\begin{array}{l}\uparrow \text { cleaved caspase-3; } \\
\downarrow \text { Akt; } \uparrow \text { MAPK; } \downarrow \text { JNK } \\
\& \text { ERK1/2 }\end{array}$ & $(64)$ \\
\hline & $\begin{array}{l}\text { 3T3-L1 mouse } \\
\text { preadipocytes }\end{array}$ & 100 & 24 & $\uparrow$ free radical scavenging & N/A & $(76)$ \\
\hline
\end{tabular}


$\uparrow$ - increase; $\downarrow$ - decrease; N/A, not available.

Acronyms: Akt, protein kinase B; AMPK $\alpha$, AMP-activated Protein Kinase $\alpha$; Bax/Bcl-2, B-cell lymphoma 2-associated X/B-cell lymphoma 2; BSO, 1-buthionine-(S,R)-sulfoximine; CREB, cAMP response element binding binding protein; CAT, chloramphenicol acetyl transferase; COX-2, cyclooxygenase-2; eNOS, endothelial nitric oxide synthase; ERK1/2, extracellular signal-regulated kinase

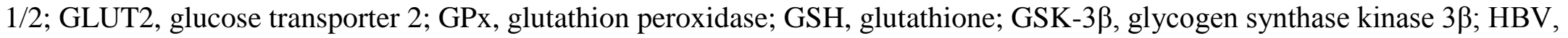
hepatitis B virus; HIV-1, human immunodeficiency virus 1; 4-HNE protein adducts, 4-hydroxynonenal-protein adducts; HO-1, heme oxygenase; IFN- $\gamma$, interferon $\gamma$; IkB, inhibitor of kappa B; IL-1 $\beta$, interleukin 1 beta; iNOS, nitric oxide synthase; IRS-1, insulin receptor substrate 1; JNK, c-Jun N-terminal kinase; LPS, lipopolysaccharides; MAGI, HeLa CD4+ HIV-1 LTR- $\beta$-gal cells; MAPK, mitogen-activated protein kinase; MMP-3, matrix metalloproteinase-3; MyD88, myeloid differentiation primary response 88; NF- $\mathrm{B}$, nuclear factor $\kappa \mathrm{B}$; NQO-1, NAD(P)H dehydrogenase; Nrf2, nuclear factor erythroid 2-related factor 2; LDL, low-density lipoprotein; PARP, poly (ADP-ribose) polymerase; PGC- $1 \alpha$, peroxisome proliferator-activated receptor- $\gamma$ coactivator $\alpha$; PGE2, prostaglandin E2; ROS, reactive oxygen species; SIRT1, sirtuin 1; SOD, superoxide dismutase; Tat, trans-activator of transcription; TNF- $\alpha$, tumor necrosis factor $\alpha$. 
Table 2.2 In vivo effects of chicoric acid in the treatment of various disorders.

\begin{tabular}{|c|c|c|c|c|c|c|c|}
\hline Diseases & $\begin{array}{l}\text { Species } \\
(\operatorname{sex})\end{array}$ & Models & $\begin{array}{l}\text { Dose } \\
(\mathrm{mg} / \mathrm{kg} / \mathrm{d})\end{array}$ & $\begin{array}{l}\text { Duration } \\
\text { (days) }\end{array}$ & Effects & $\begin{array}{l}\text { Suggested } \\
\text { Mechanisms }\end{array}$ & References \\
\hline \multirow[t]{5}{*}{$\begin{array}{l}\text { Brain } \\
\text { function }\end{array}$} & $\begin{array}{l}\text { Swiss albino } \\
\text { mice }(\mathrm{M})\end{array}$ & $\begin{array}{l}\text { Restraint stress } \\
\text { (Porsolt's swim } \\
\text { stress \& conical } \\
\text { polypropylene } \\
\text { tubes) }\end{array}$ & $1-2$ p.o. & 14 & $\begin{array}{l}\downarrow \text { behavioral } \\
\text { despair; } \\
\uparrow \text { learning ability; } \\
\uparrow \text { neurotransmitters }\end{array}$ & $\begin{array}{l}\uparrow \text { norepinephrine; } \\
\uparrow \text { dopamine; } \uparrow 5 \text { - } \\
\text { hydroxy } \\
\text { tryptamine }\end{array}$ & $(66)$ \\
\hline & $\begin{array}{l}\text { Sprague-Dawley } \\
\text { rats \& ICR mice } \\
\text { (M) }\end{array}$ & $\begin{array}{l}\text { Retinal damage } \\
\text { (N-methyl-D- } \\
\text { aspartate / optic } \\
\text { nerve crush) }\end{array}$ & $\begin{array}{l}2 \mu \mathrm{g} / \text { eye } \\
\text { intravitreal } \\
\text { injection }\end{array}$ & 7 & $\begin{array}{l}\uparrow \text { retinal ganglion } \\
\text { cell viability }\end{array}$ & $\begin{array}{l}\downarrow \text { cleaved PARP; } \downarrow \\
\text { cleaved caspase-3 }\end{array}$ & $(67)$ \\
\hline & $\begin{array}{l}\text { C57BL/6J mice } \\
(\mathrm{M})\end{array}$ & LPS challenges & $\begin{array}{l}0.5 \mathrm{~g} / \mathrm{L} \\
\text { drinking } \\
\text { water }\end{array}$ & 45 & $\begin{array}{l}\downarrow \text { memory } \\
\text { impairment; } \downarrow \\
\text { amyloidogenesis }\end{array}$ & $\begin{array}{l}\downarrow \text { amyloid } \beta\left(\mathrm{A} \beta_{1-}\right. \\
42) ; \\
\downarrow \text { BACE } 1 ; \downarrow \\
\text { MAPK; } \downarrow \text { NF- } \kappa \mathrm{B}\end{array}$ & (33) \\
\hline & $\begin{array}{l}\text { C57BL/6J mice } \\
(\mathrm{M})\end{array}$ & LPS challenges & $\begin{array}{l}0.5 \mathrm{~g} / \mathrm{L} \\
\text { drinking } \\
\text { water }\end{array}$ & 45 & $\begin{array}{l}\downarrow \text { oxidative stress } \\
\text { induced neuron } \\
\text { damage }\end{array}$ & $\begin{array}{l}\downarrow \text { MAPK; } \downarrow \text { NF- } \\
\kappa B ; \downarrow \text { iNOS; } \downarrow \text { IL- } \\
1 \beta ; \downarrow \text { TNF- } \alpha ; \uparrow \\
\text { Nrf2; } \uparrow \text { HO- } 1 ; \uparrow \\
\text { NQO-1 }\end{array}$ & (34) \\
\hline & $\begin{array}{l}\text { C57BL/6J mice } \\
(\mathrm{M})\end{array}$ & $\begin{array}{l}\text { D-glactose } \\
\text { challenges }\end{array}$ & 100 i.p. & 56 & $\begin{array}{l}\downarrow \text { neuron damage; } \\
\downarrow \text { hippocampus } \\
\text { shrinkage }\end{array}$ & $\begin{array}{l}\downarrow \text { amyloid } \beta\left(\mathrm{A} \beta_{1-}\right. \\
42) ; \uparrow \mathrm{BDNF}\end{array}$ & $(75)$ \\
\hline \multirow[t]{2}{*}{$\begin{array}{l}\text { Glucose } \\
\text { metabolism }\end{array}$} & Wistar rats $(\mathrm{M})$ & No challenge & 3-30 i.p. & 4 & $\begin{array}{l}\uparrow \text { insulin secretion; } \\
\downarrow \text { hyperglycemia }\end{array}$ & N/A & $(50)$ \\
\hline & Swiss mice $(\mathrm{M})$ & $\begin{array}{l}\text { Streptozotocin } \\
\text { challenge }\end{array}$ & 3 i.p. & $2 \mathrm{hrs}$ & $\downarrow$ hyperglycemia & N/A & $(59)$ \\
\hline
\end{tabular}




\begin{tabular}{|c|c|c|c|c|c|c|c|}
\hline & Swiss mice $(\mathrm{M})$ & $\begin{array}{l}\text { Streptozotocin } \\
\text { challenge }\end{array}$ & $\begin{array}{l}1-3 \\
\text { i.p. }\end{array}$ & $2 \mathrm{hrs}$ & $\downarrow$ hyperglycemia & N/A & $(60)$ \\
\hline & $\begin{array}{l}\text { C57BL/6J mice } \\
(\mathrm{M})\end{array}$ & $\begin{array}{l}\text { Streptozotocin } \\
\text { challenge }\end{array}$ & $\begin{array}{l}60 \text { drinking } \\
\text { water }\end{array}$ & 4 & $\begin{array}{l}\downarrow \text { hyperglycemia; } \downarrow \\
\text { pancreas } \\
\text { apoptosis; } \uparrow \\
\text { insulin secretion }\end{array}$ & $\begin{array}{l}\downarrow \mathrm{JNK} ; \downarrow \text { Bax/Bcl- } \\
2 ; \uparrow \mathrm{PDX}-1\end{array}$ & $(61)$ \\
\hline \multirow[t]{4}{*}{$\begin{array}{l}\text { Lipid } \\
\text { metabolism } \\
\text { \& liver } \\
\text { function }\end{array}$} & $\begin{array}{l}\text { C57BL/6J mice } \\
\text { (M) }\end{array}$ & $\begin{array}{l}\text { High-fat diet } \\
\text { challenge }\end{array}$ & 15-60 р.о. & 8 & $\begin{array}{l}\downarrow \text { body weight; } \downarrow \\
\text { hepatic steatosis }\end{array}$ & $\begin{array}{l}\downarrow \text { TNF- } \alpha ; \downarrow \text { IL-6; } \downarrow \\
\text { COX-2; } \downarrow \text { JNK; } \downarrow \\
\text { PPAR } \gamma ; \downarrow \\
\text { C/EBP } \alpha ; \downarrow \text { FAS; } \downarrow \\
\text { ALT \& AST }\end{array}$ & $(64)$ \\
\hline & $\begin{array}{l}\text { C57BL/6J mice } \\
\text { (F) }\end{array}$ & $\begin{array}{l}\text { Hepatic steatosis } \\
\text { (alcohol) }\end{array}$ & $\begin{array}{l}4 \text { drinking } \\
\text { water }\end{array}$ & 4 & $\begin{array}{l}\downarrow \text { hepatic } \\
\text { triacylglycerols }\end{array}$ & $\begin{array}{l}\downarrow \text { iNOS } ; \downarrow \text {-HNE } \\
\text { protein adducts; } \downarrow \\
\text { TNF- } \alpha ; \downarrow \text { PAI- } ; \downarrow \downarrow \\
\text { CD11c }\end{array}$ & $(65)$ \\
\hline & $\begin{array}{l}\text { C57BL/6J mice } \\
(\mathrm{M})\end{array}$ & $\begin{array}{l}\text { Methionine- and } \\
\text { choline-deficient } \\
\text { diet challenge }\end{array}$ & $10-30$ p.o. & 4 & $\begin{array}{l}\downarrow \text { hepatic lipid } \\
\text { accumulation; } \downarrow \\
\text { lipid peroxidation; } \\
\downarrow \text { hepatic } \\
\text { ballooning, } \\
\text { steatosis \& } \\
\text { inflammation }\end{array}$ & $\begin{array}{l}\downarrow \text { SREBP-1c; } \uparrow \\
\text { Nrf2; } \uparrow \text { AMPK; } \downarrow \\
\text { TNF- } \alpha ; \downarrow \text { MCP-1; } \\
\downarrow \text { FAS } \downarrow \downarrow \text { ALT \& } \\
\text { AST }\end{array}$ & (63) \\
\hline & $\begin{array}{l}\text { C57BL/6J mice } \\
\text { (M) }\end{array}$ & $\begin{array}{l}\text { Streptozotocin } \\
\text { challenge }\end{array}$ & $\begin{array}{l}60 \text { drinking } \\
\text { water }\end{array}$ & 28 & $\downarrow$ hepatic injury & $\begin{array}{l}\uparrow \text { glycogen; } \uparrow \\
\text { glycolysis genes } \\
\text { (Gck, Pk \& Pfk); } \uparrow \\
\text { AMPK }\end{array}$ & $(52)$ \\
\hline Inflammation & Sprague-Dawley & LPS challenge & 20 p.o. & 4 & $\uparrow$ phagocytic & $\uparrow$ TNF- $\alpha ; \uparrow$ IFN- $\gamma$ & $(45)$ \\
\hline
\end{tabular}




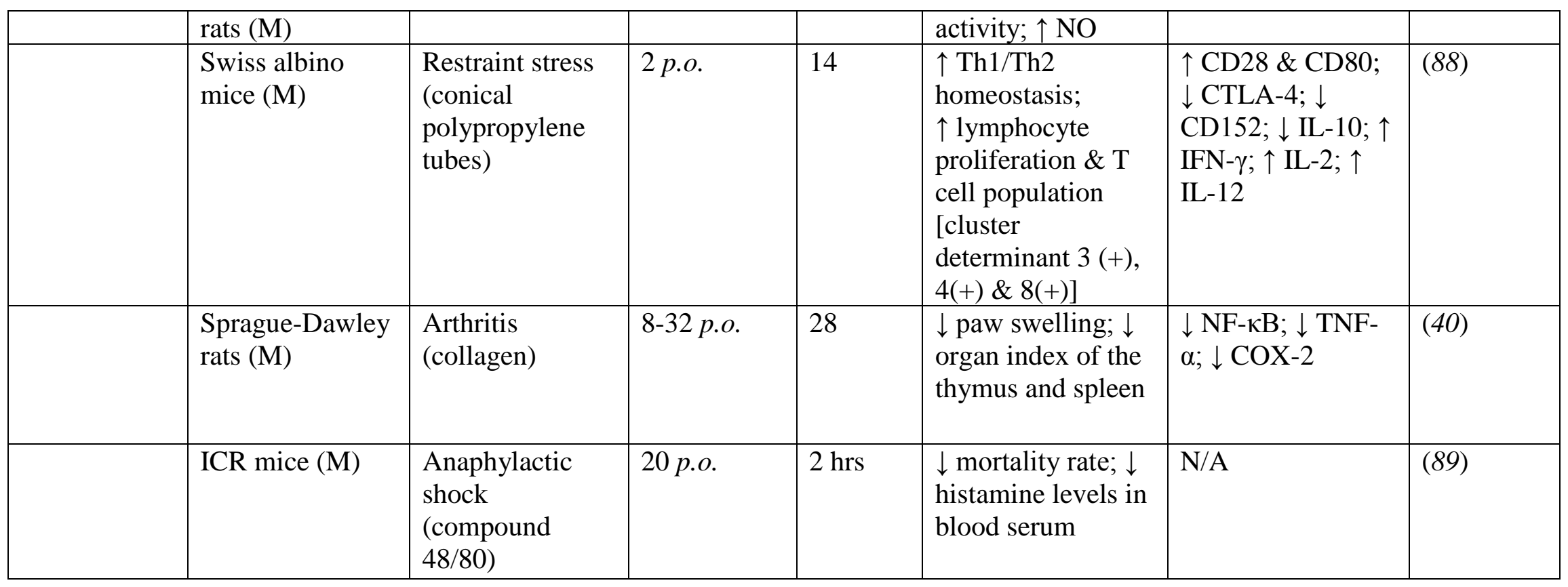

M - male; F - female; $\uparrow$ - increase; $\downarrow$ - decrease; N/A, not available; p.o., Per os (oral administration); i.p., intraperitoneal injection. Acronyms: Akt, protein kinase B; ALT, alanine aminotransferase; AMPK $\alpha$, AMP-activated Protein Kinase $\alpha$; AST, aspartate aminotransferase; BACE1, neuronal $\beta$-secretase 1; Bax/Bcl-2, B-cell lymphoma 2-associated X/B-cell lymphoma 2; BDNF, brainderived neurotrophic factor; CD11c, integrin $\alpha$ X chain protein; CD28, cluster of differentiation 28; CD80, cluster of differentiation 80; $\mathrm{CD} 152$, cluster of differentiation 152; C/EBP $\alpha, \mathrm{CCAAT} /$ enhancer binding protein $\alpha$; COX-2, cyclooxygenase-2; FAS, fatty acid synthase; CTLA-4, cytotoxic T-lymphocyte associated antigen 4; 4-HNE protein adducts, 4-hydroxynonenal-protein adducts; HO-1, heme oxygenase; IFN- $\gamma$, interferon $\gamma$; IL-1 $\beta$, interleukin $1 \beta$; iNOS, nitric oxide synthase; JNK, c-Jun N-terminal kinase; Keap1, kelchlike ECH-associated protein 1; LPS, lipopolysaccharides; MAPK, mitogen-activated protein kinase; MCP-1, monocyte chemoattractant protein 1 ; NF- $\kappa \mathrm{B}$, nuclear factor $\kappa \mathrm{B}$; Nrf2, nuclear factor erythroid 2-related factor 2; NQO-1, NAD(P)H dehydrogenase; PAI-1, plasminogen activator inhibitor-1; PARP, poly (ADP-ribose) polymerase; PDX-1, pancreatic duodenal 
homeobox 1; PPAR $\gamma$, peroxisome proliferator activated receptor $\gamma$; ROS, reactive oxygen species; SREBP-1, sterol regulatory element-binding protein 1 ; TNF- $\alpha$, tumor necrosis factor $\alpha$; Th1/Th2, T helper cells $1 / 2$. 


\subsection{Suggested molecular targets of chicoric acid}

As discussed in previous sections, chicoric acid modulates genes, transcription factors, growth factors, enzymes, and proteins involved in important cellular processes, such as virus infection, chemoresistance, inflammation, and glucose metabolism (Figure 2.2) $(11,42,82,85)$. Among them, it is suggested that NF- $\kappa B$ and TNF- $\alpha$ are two major mediators of chicoric acid's activities.

$\mathrm{NF}-\kappa \mathrm{B}$ is a protein complex involved in cellular responses to stress, free radicals, heavy metals, and bacterial or viral infections through regulating DNA transcription, cytokine production, and cell survival $(37,38,90)$. It is known that the activity of NF- $\mathrm{B}$ is directly suppressed by binding with the inhibitor of $\kappa \mathrm{B}(\mathrm{I} \kappa \mathrm{B})$ in the cytoplasm $(90)$. In

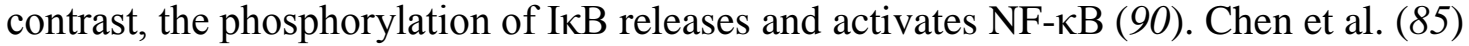
reported that chicoric acid deactivates $\mathrm{NF}-\kappa \mathrm{B}$ by inhibiting the phosphorylation of $\mathrm{I} \kappa \mathrm{B}$, indicating that $\mathrm{I} \kappa \mathrm{B}$ may be a major regulator for chicoric acid-mediated inactivation of $\mathrm{NF}-\kappa \mathrm{B}$. Alternatively, NF- $\kappa \mathrm{B}$ can be activated in combination with TNF- $\alpha$ (37). Several studies reported that chicoric acid decreased TNF- $\alpha$ production $(40,47,64,65,75)$, which might be a contributing factor to the reduced activation of NF- $\kappa \mathrm{B}$. Chicoric acidrelated deregulations of NF- $\mathrm{BB}$ and TNF- $\alpha$ were shown to alleviate conditions such as autoimmune disorders, restraint stress, hepatic steatosis and neuron damage known to be involved in cellular inflammation and associated diseases $(34-36,40,47,63-65,75)$. 


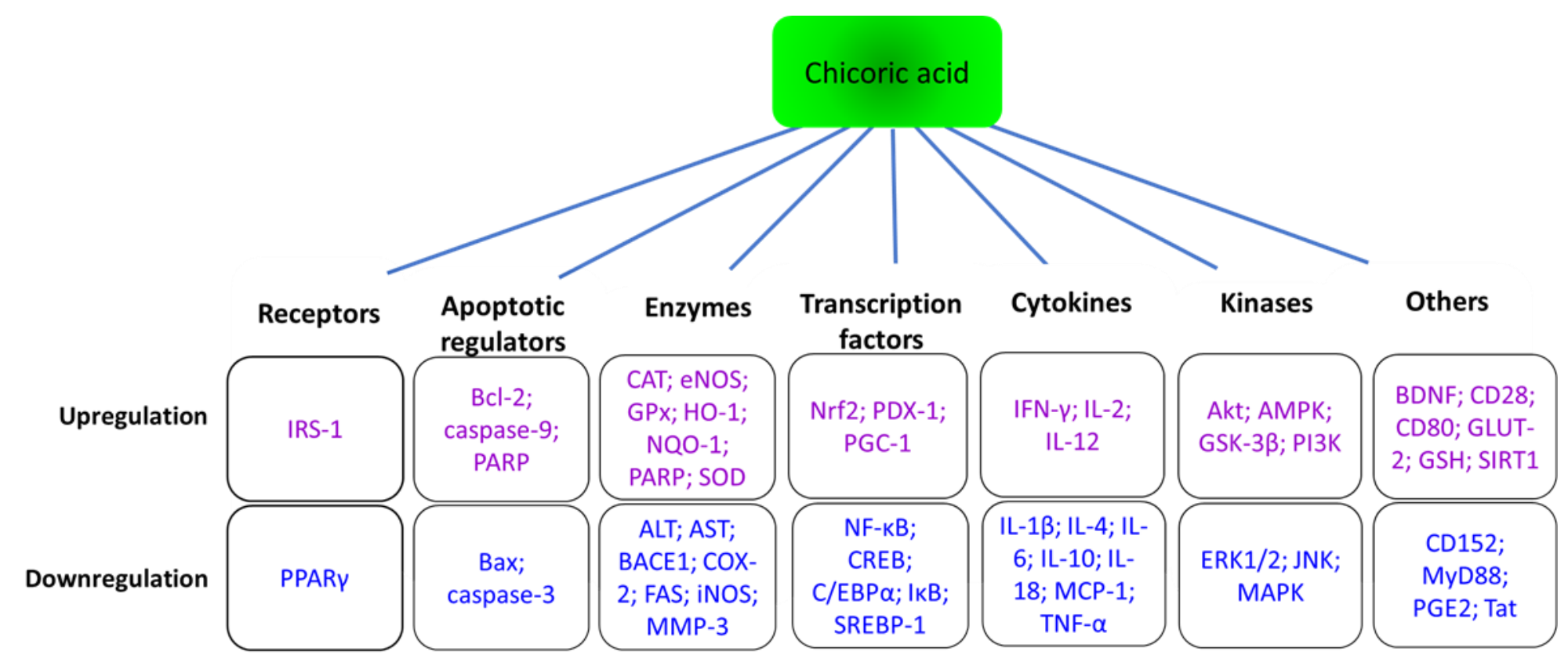

Figure 2.2 Molecular targets of chicoric acid. Akt, protein kinase B; ALT, alanine aminotransferase; AMPK $\alpha$, AMP-activated Protein Kinase $\alpha$; AST, aspartate aminotransferase; BACE1, neuronal $\beta$-secretase 1; Bax/Bcl-2, B-cell lymphoma 2-associated X/B-cell lymphoma 2; BDNF, brain-derived neurotrophic factor; CD28, cluster of differentiation 28; CD80, cluster of differentiation 80; $\mathrm{CD} 152$, cluster of differentiation 152; $\mathrm{C} / \mathrm{EBP} \alpha$, CCAAT/enhancer binding protein $\alpha$; COX-2, cyclooxygenase-2; ERK1/2, extracellular signal-regulated kinase 1/2; FAS, fatty acid synthase; HO-1, heme oxygenase; GLUT2, glucose transporter 2; IFN- $\gamma$, interferon $\gamma$; IL-1 $\beta$, interleukin $1 \beta$; iNOS, nitric oxide synthase; JNK, c-Jun N-terminal kinase; MAPK, mitogen-activated protein kinase; MCP-1, monocyte chemoattractant protein 1; NF- $\kappa \mathrm{B}$, nuclear factor $\kappa \mathrm{B}$; Nrf2, nuclear factor erythroid 2-related factor 2; NQO-1, NAD(P)H dehydrogenase; PARP, poly (ADP-ribose) polymerase; PDX-1, pancreatic duodenal homeobox 1; PPAR $\gamma$, peroxisome proliferator activated receptor $\gamma$; SREBP-1, sterol regulatory element-binding protein 1; TNF- $\alpha$, tumor necrosis factor $\alpha$; Th1/Th2, T helper cells $1 / 2$. 


\subsection{Pharmacokinetic study of chicoric acid}

Chicoric acid is known to have relatively low absorption in rats (91). The oral administration of chicoric acid at $50 \mathrm{mg} / \mathrm{kg}$ body weight resulted in a peak plasma concentration of chicoric acid at $1.63 \pm 0.25 \mathrm{mg} / \mathrm{L}$ after $4 \mathrm{~h}(91)$. Chicoric acid was mainly distributed in the liver, lung and kidney after oral administration for $3 \mathrm{~h}$. Chicoric acid has a relatively long residence time and low body clearance, $18.58 \pm 4.43 \mathrm{~h}$ and 2.80 $\pm 0.46 \mathrm{~L} / \mathrm{kg} / \mathrm{h}$, respectively, in rats $(91)$. All these findings help in better understanding the bioavailability and fate of chicoric acid.

\subsection{Conclusion}

The prevention of early-stage chronic diseases by food bioactive seems to be a promising strategy. Chicoric acid's multi-bioactivities suggest it has great potential in treating a number of metabolic disorders, including inflammatory responses, impairment of energy homeostasis, brain dysfunction, and immune disorders. Even though there are increasing number of studies reporting bioactivities of chicoric acid, these are still limitations to make a concrete conclusion due to differences on models, doses, and treatment durations used, as well as lack of pharmacokinetic study, including metabolism, of chicoric acid in humans. The research on chicoric acid is still at an early stage, so there are still many questions that need to be answered regarding its benefits to health. Thus, more studies are needed to guide the development of chicoric acid-based functional food products. 


\section{CHAPTER 3}

\section{OBJECTIVES OF THE PROJECT}

The long-term goal is to develop chicoric acid as a functional food bioactive for the prevention and/or treatment for aging and type 2 diabetes. Previous studies reported that chicoric acid extends C. elegans lifespan and promotes glucose transport in hepatic cells (7). However, more experiments are needed to further investigate the effects of chicoric acid on aging-related phenotypes in C. elegans and the glucose uptake in other models. The object of this project is to determine the effect of chicoric acid on the agingassociated phenotypes of C. elegans and glucose uptake in muscle cells. The central hypothesis is that chicoric acid can protect $C$. elegans during aging as well as promote glucose uptake in muscle cells. The rationale of the proposed research is that by investigating the effect of chicoric acid on aging and glucose uptake as well as the underlying mechanisms, we will be able to better understand chicoric acid as a beneficial bioactive for the promotion of health span and prevention from type 2 diabetes.

The project aims are as follows:

Specific aim 1: Determine the effect of chicoric acid on aging and aging-related phenotypes of $C$. elegans. The hypothesis is that chicoric acid extends the lifespan of $C$. elegans and delays the decrease of aging-related phenotypes.

Specific aim 2: Investigate the effect of chicoric acid on glucose uptake in muscle cells and mice. The hypothesis to be tested is that chicoric acid promotes the glucose uptake in $\mathrm{C} 2 \mathrm{C} 12$ myotubes and reduces the blood glucose level in mice. 


\section{CHAPTER 4}

\section{EFFECTS OF CHICORIC ACID ON AGING AND \\ GLUCOSE UPTAKE}

\subsection{Effect of chicoric acid on aging in C elegans and the underlying mechanisms}

\subsubsection{Introduction}

Aging is defined as a progressive decline of intrinsic physiological functions, resulting in an increased risk of death (5). Considering that aging is one of the key risk factors associated with many chronic diseases, slowing aging is of great importance to prevent age-associated diseases and improve the health span of life (6). Currently, foodderived bioactive compounds are drawing significant attention because of their anti-aging effects and the modulation of aging-related pathways (92-95), which provide potentially valuable preventive tools for aging and aging-related disorders.

Chicoric acid, one of the hydroxycinnamic acids, is a dicaffeoyl ester naturally found in the roots of many plants, such as chicory, purple coneflower, and basil (4). Chicoric acid is reported to have a number of bioactivities, including antioxidation, antiinflammation, antivirus and immune-stimulating properties through multiple molecular pathways $(18,33,41,52)$. A previous study reported that chicoric acid extended the lifespan in Caenorhabditis elegans (7), however, the effects of chicoric acid on agingrelated phenotypes and the mechanisms underlying these effects remain to be determined.

One recent study reported that chicoric acid regulates glucose metabolism via AMP-activated protein kinase (AMPK) activation (52). AMPK is an energy sensor that controls energy homeostasis through the regulation of AMP/ATP metabolism in cells (4). 
Moreover, AMPK is considered important in the regulation of longevity as the increased activation of AMPK extended the lifespan through dietary restriction and/or enhanced stress resistance in C. elegans and Drosophila melanogaster (96, 97). aak-2, a homolog of the $\alpha$-catalytic subunits of mammalian AMPK, is evolutionarily conserved in both $C$. elegans and mammals (98). Since AMPK has been reported to be activated by chicoric acid (52), it is plausible to assume that chicoric acid might regulate the lifespan of $C$. elegans via aak-2. In addition to aak-2, skn-1, encoding a homolog of nuclear factor erythroid 2-related factor 2 (Nrf-2) in C. elegans, was also reported to extend lifespan by regulating the oxidative stress response pathway (99). Therefore, the current research focused on determining the influence of chicoric acid on aging-related phenotypes in $C$. elegans and the role of AAK-2 and SKN-1 in this process.

\subsubsection{Materials and methods}

\subsubsection{Materials}

Chicoric acid ( $\geq 98 \%$ ) was purchased from PufeiDe Biotech Co., Ltd (Chengdu, Sichuan, China). Household bleach used for bleaching adult nematodes during synchronization was obtained from Clorox company (Oakland, CA, USA). Fluorodeoxyuridine (FUdR), ampicillin, and carbenicillin, paraquat, and 2',7'dichlorodihydrofluorescein diacetate (DCFDA) were ordered from Sigma-Aldrich (St. Louis, MO, USA). Rabbit antibodies for phospho-AMPK and $\alpha$-tubulin, as well as goat anti-rabbit IgG-horseradish peroxidase (HRP) were obtained from Cell Signaling Technology (Danvers, MA, USA). Rabbit antibodies for phospho-AMPK and $\alpha$-tubulin, 
as well as goat anti-rabbit IgG-horseradish peroxidase (HRP), were obtained from Cell Signaling Technology (Danvers, MA, USA).

\subsubsection{C. elegans: strains and maintenance}

The strains in the study were provided by the Caenorhabditis Genetics Center (CGC, University of Minnesota, Minneapolis, MN, USA): wild-type Bristol N2, aak-2 (ok524) X, daf-2 (e1370) III, eat-2(ad1116) II, daf-16(mgDf50) I, skn-1(zu135) IV, agel(hx546), and ldIs 7 (skn-lb/c::gfp; rol-6). All strains were grown and incubated at $20{ }^{\circ} \mathrm{C}$ on nematode growth media (NGM) plates with Escherichia coli OP50, except daf-2, which was maintained at $15^{\circ} \mathrm{C}$. The solutions including nematode growth medium (NGM), M9 buffer, and S-complete required for C. elegans culture were prepared according to the worm book (100). A synchronized culture was obtained as previously described (101). The chicoric acid stock solutions with 12.5, 25, and $50 \mathrm{mM}$ were prepared using dimethyl sulfoxide (DMSO) as solvent. Stock solutions were diluted 1000 times resulting in final concentrations of chicoric acid at $12.5,25$, and $50 \mu \mathrm{M}$. These doses were based on a previous report on the effects of chicoric acid on life span extension in C. elegans (7). For lifespan analysis, L4 stage nematodes were transferred in Transwell-96 well plate (Corning Inc., NY, USA; n=10-15 worms per well, 15-18 wells/ treatment) and treated with $0.1 \%$ DMSO (control) or chicoric acid during the lifespan study $(94,102,103)$. FUdR $(120 \mathrm{mM})$, to prevent eggs from hatching out, was also added (104). The medium was changed every four days. The survivals were determined by a gentle touch-prod with a sterilized picker under a microscope (Nikon Instruments Inc., Melville, NY, USA) $(94,103)$. Worm viability was recorded every other day until all 
worms died. The day that the treatment began is considered as day 0 . The lifespan test in $\mathrm{N} 2$ was repeated for three independent trials.

\subsubsection{Growth rate, body size, and moving speed determination}

The growth rate of C. elegans was determined by quantifying the population of worms at three different developmental stages after $48 \mathrm{~h}$ chicoric acid treatments. Results were shown in percent of proportion of C. elegans in each stage (94). Body size and moving speed of C. elegans were measured by the WormLab tracking system (Allied Vision Technologies, Stadtroda, Germany) and analyzed by WormLab Software (MBF Bioscience, Williston, VT, USA) as published (101). Worms were transferred to the lowpeptone NGM plates seeded with OP50 and were undisturbed for 30 min for acclimation before tracking (94). A 1-min recording (8.12 frames per second) was captured. The size of the worms (70-90/treatment) was measured by the WormLab Software after $48 \mathrm{~h}$ of treatments. The moving speed of worms was measured every other day from day 2 to day 12.

\subsubsection{Progeny production and pumping rate determination}

After $48 \mathrm{~h}$ chicoric acid treatments, two L4 staged nematodes/treatment were shifted to freshly prepared NGM plates with E. coli OP50 every day during the reproduction period. The number of progeny hatching out of eggs was counted and recorded daily (94). The pumping rate was obtained by quantifying pharyngeal contraction times of nematodes using a microscope (Olympus Corporation, Tokyo, Japan) 
for $30 \mathrm{sec}$. The test was repeated three times with 12 randomly-selected nematodes per treatment (94).

\subsubsection{Measurement of intracellular ROS and oxidative stress resistance}

The intracellular reactive oxygen species (ROS) level was detected using DCFDA, a fluorescent probe commonly applied for ROS detection (105). After a 72-h treatment with DMSO (control group) or chicoric acid, day 1 adult nematodes were washed with M9 buffer three times to remove bacteria. They were next transferred into a 96-well plate, $\sim 60$ worms/well, with or without chicoric acid treatments in $50 \mu \mathrm{L}$ of M9 buffer, following the mixture of $50 \mu \mathrm{L}$ DCFDA solution $(200 \mu \mathrm{M})$ to achieve $100 \mu \mathrm{M}$ of the final concentration. A well with $100 \mu \mathrm{M}$ DCFDA solution with no worms was used as the background, and worms treated with DCFDA solution and $5 \mathrm{mM}$ paraquat were used as the positive control. The fluorescent readings were taken after $30 \mathrm{~min}$ of incubation at an emission/excitation wavelength of 530 and $485 \mathrm{~nm}$ by an Infinite 200 PRO microplate reader (Tecan Trading AG, Switzerland).

For oxidative stress resistance determination, day 1 adult worms were transferred into the 96 trans-well plate, $12-15$ worms per well, containing $5 \mathrm{mM}$ paraquat, an oxidative stress inducer. The oxidative stress resistance of $\mathrm{C}$. elegans was determined by counting the survival percentage of worms under the exposure of paraquat for 2, 4, and 6 days with or without chicoric acid treatment (i.e., $25 \mu \mathrm{M})(94)$.

\subsubsection{Quantitative real-time PCR}

RNA was extracted in each sample using Trizol under RNase-free conditions, as 
previously described (101). cDNA was prepared by reverse transcription of mRNA using a high-capacity cDNA reverse transcription kit (Thermo Fisher Scientific Inc, Middletown, VA, USA). The gene expression was quantified by QuantStudio 3 real-time PCR system using the TaqMan detection assay (Applied Biosystems, Waltham, MA, USA). $a a k-2$ (Ce02404259_g1), nhr-49 (Ce02412666_g1), skn-1 (Ce02407444_m1), gsr1 (Ce02444754_g1) and pbs-5 (Ce02426103_g1), and the internal control ama-1

(Ce02462726_m1) were ordered from Thermo Scientific (Middletown, VA, USA).

\subsubsection{Intracellular localization of SKN-1::GFP}

Transgenic strain $l d I s 7(s k n-1 b / c:: g f p$; rol-6) was used to detect the intracellular localization of GFP tagged SKN-1 protein. After two-day of chicoric acid treatment (25 $\mu \mathrm{M}$ ), around 10-20 worms (L4 stage or young adults) were placed on each microscope slide coated with $2 \%$ agarose pad and anesthetized with $10 \mathrm{mM} \mathrm{NaN}_{3}$ within 3 min (94). Worms treated with NaN3 (2\%) for 15 min were used as the positive control. The cellular localizations of SKN-1 were detected by a Nikon Eclipse Ti-U (Nikon Instruments Inc., Melville, NY, USA). The nuclear translocation patterns of SKN-1::GFP were identified as 'low', 'medium', and 'high'. 'Low' refers to worms with almost no visible nuclear translocation; 'medium' refers to C. elegans with nuclear translocation in the partial body; 'high' refers to worms with nuclear translocation throughout the whole body, as shown in Figure 4.6D. Worms were counted and analyzed as percentages of nuclear translocation including both 'medium' and 'high' translocation (103).

\subsubsection{Western blotting}


The C. elegans protein extraction was obtained according to the protocol published with minor modification $(101,106)$. Worms were collected and washed four times with M9 buffer to remove bacteria. Then worms (approximately 6000 young adults per sample) were suspended by $500 \mu \mathrm{L}$ PBS mixed with protease and phosphatase inhibitors (Beyotime Biotechnology, Shanghai, China). Samples were homogenized and centrifuged $(12,000 \times \mathrm{g})$ at $4{ }^{\circ} \mathrm{C}$ for $10 \mathrm{~min}$ to harvest the supernatants. After protein normalization, the samples with $5 \times$ sample buffer were electrophoresed on a $10 \%$ SDS polyacrylamide gel and transferred onto a polyvinylidine fluoride membrane (107). After blocking with 5\% skim milk, the membrane was incubated with rabbit monoclonal antiphospho-AMPK and rabbit anti- $\alpha$-tubulin antibody. Horseradish peroxidase-conjugated goat anti-rabbit IgG was used as the secondary antibody. Protein blots were pictured by a Tanon 5500 image station (Shanghai, China) with ECL Substrate Kit (Bio-Rad Co., Hercules, CA, USA). Images were quantified with ImageJ software.

\subsubsection{Statistical analysis}

Survival curves were analyzed by Log-rank (Mantel-Cox) tests by Graph Pad Prism version 6.0 (GraphPad Software, Inc., San Diego, CA, USA). The data of nuclear translocation of SKN-1::GFP in Fig. 4.6D was analyzed by chi square tests by SAS software (version 9.3, SAS Institute Inc., Cary, NC, USA). For other data, differences between each treatment were analyzed using one-way ANOVA (Tukey's multiple-range test, SAS). Significance of differences was defined as $P$ values of $<0.05$.

\subsubsection{Results}




\subsubsection{Chicoric acid extended the lifespan of $C$. elegans}

Compared with the control, chicoric acid at 25 and $50 \mu \mathrm{M}$, but not $12.5 \mu \mathrm{M}$, significantly extended the lifespan of wild type nematodes ( $P<0.0001$ for both; Fig. 1). There was no difference in lifespan between 25 and $50 \mu \mathrm{M}$ chicoric acid treatments. Table 1 also exhibited the extended median lifespan of wild type N2 worms with 25 and $50 \mu \mathrm{M}$ chicoric acid treatment, compared to the control. This was consistently observed from 3 independent experiments: (median lifespan as days) $18.7 \pm 0.3$ for control, $23.3 \pm 0.9$ for $25 \mu \mathrm{M}$ chicoric acid, and $22.7 \pm 0.7$ for $50 \mu \mathrm{M}$ chicoric acid with $\mathrm{P}=0.0062$ and $\mathrm{P}=0.0128$ for 25 and $50 \mu \mathrm{M}$ chicoric acid compared to the control, respectively.

Thus, 25 and $50 \mu \mathrm{M}$ were used to determine the aging-related phenotypes.

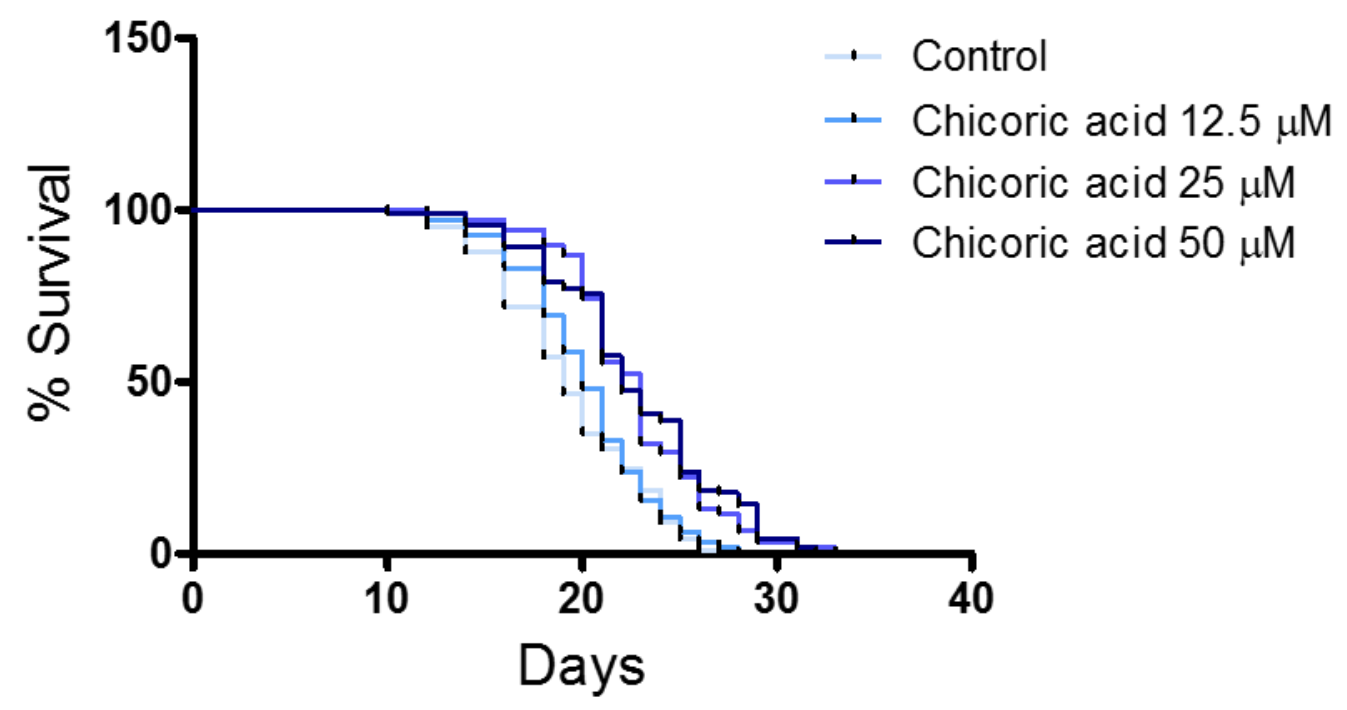

Figure 4.1 Survival of wild-type C. elegans treated with chicoric acid. C. elegans (N2) were treated with final concentrations of 0 (control), $12.5,25$, or $50 \mu \mathrm{M}$ chicoric acid starting from L4 stage (day 0 ). The survivals were recorded every other day until all the worms died ( $\mathrm{n}=206-266 /$ treatment). Log-rank (Mantel-Cox) test was used for statistical analysis. Significant differences of median/maximum lifespan compared between the control and the treatment groups were marked as $* * * P<0.001$.

The physiological properties of C. elegans treated by chicoric acid after 
synchronization were determined by growth rate, body size, and progeny (Fig. 4.2). After $48 \mathrm{~h}$ of treatment, $76-80 \%$ of the nematodes reached L4 stage or young adult stage, while 20-23\% were at early/mid L4 stage (Fig. 4.2A). As shown in Fig. 4.2, no difference was observed in the growth rate, worm length, worm width, or progeny number of C. elegans treated with or without chicoric acid. The results suggest that the physiological properties of nematodes were not affected by chicoric acid.
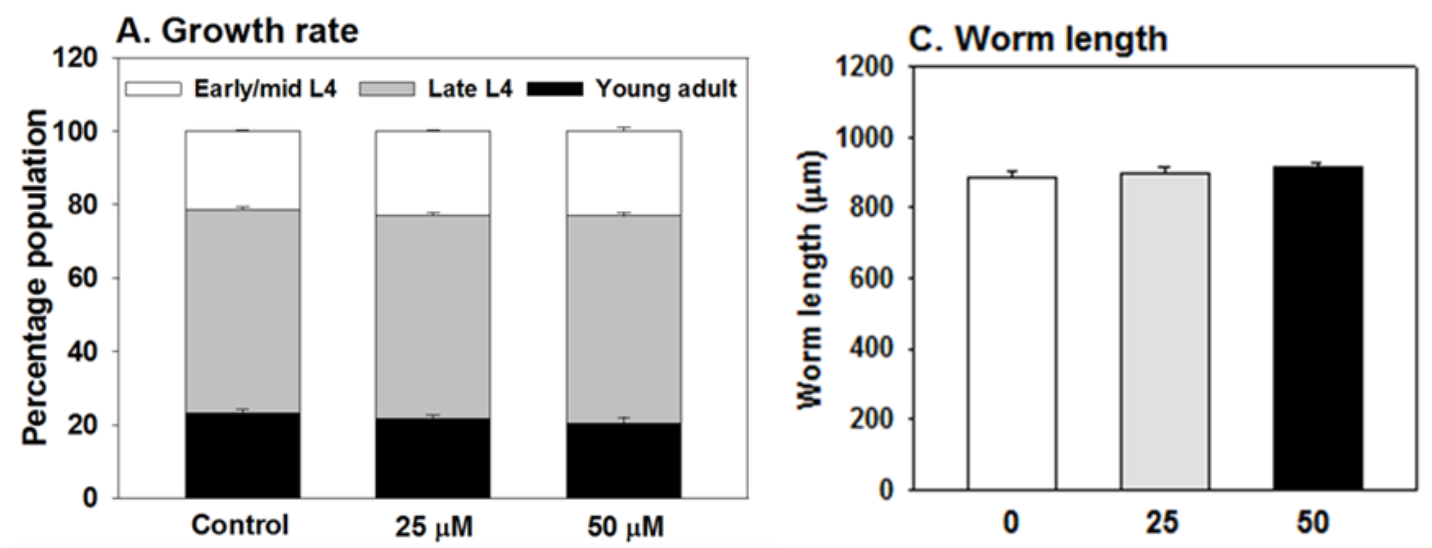

B. Progeny production

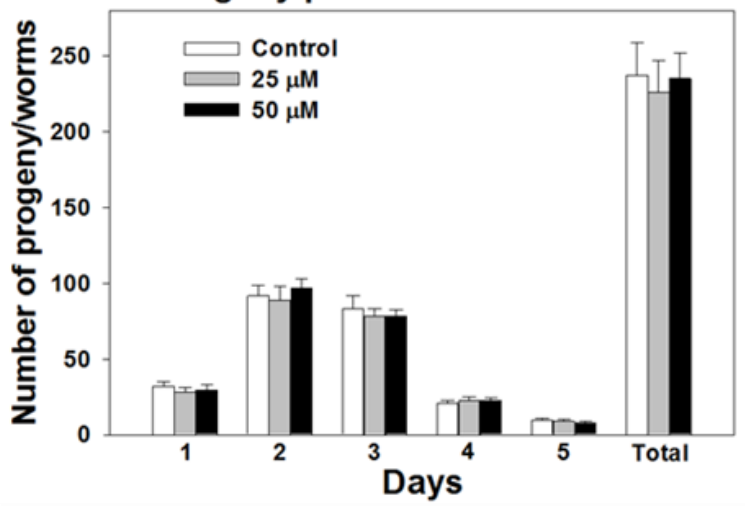

D. Worm width

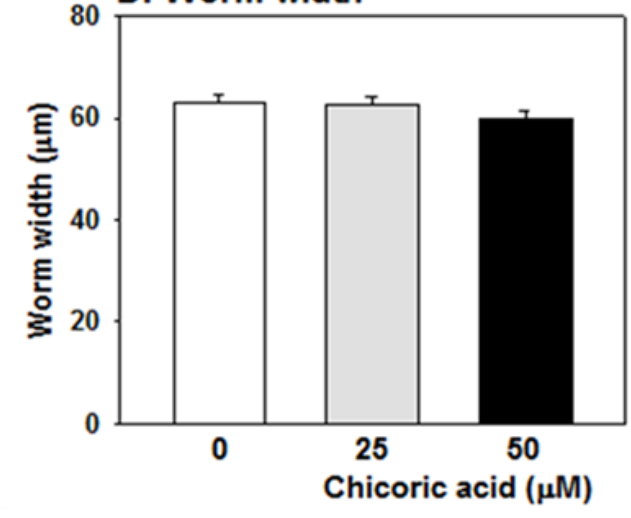

Figure 4.2 Effect of chicoric acid on growth rate, worm size and progeny production of C. elegans. (A) Synchronized L1 worms were raised on NGM plates with 0, 25 and 50 $\mu \mathrm{M}$ chicoric acid. After $48 \mathrm{~h}$ treatments, the number of worms at three developmental stages was counted and calculated in percent of proportion. (B) Synchronized L1 larval worms were treated with chicoric acid and the number of progeny hatching out from day 1 to day 5 were recorded. (C) Worm length and (D) width were measured by WormLab software after $48 \mathrm{~h}$ treatments from the $\mathrm{L} 1$ stage. Values are means \pm S.E. $(\mathrm{n}=3$ plates and $\sim 150$ worms/plate for growth rate; $\mathrm{n}=3$ plates and 2 worms/plate for progeny production, $\mathrm{n}=140-180$ for worm size). 


\subsubsection{Effect of chicoric acid on the age-associated decline of phenotypes and ROS levels}

Two typical markers of aging-relative phenotypes in C. elegans are pharyngeal pumping rate and locomotive activity (102). Thus, we next detected the effects of chicoric acid on these two markers (Fig. 4.3). The pumping rate as expected declined progressively with aging: $60 \%$ decline of the pumping rate in the control group from day 4 to day 12 (Fig. 4.3). Chicoric acid treatments with both 25 and $50 \mu \mathrm{M}$ statistically delayed the decrease of pumping rate compared to the control (Fig. 4.3A). A significant difference was first observed on day 6 , and a greater difference was found on day 12 (29.1\% and 32.9\% improvement, respectively, compared to the control). Aging resulted in the decline of moving speed by $76.4 \%$ on day 12 , compared with that of day 2 in the control (Fig. 4.3B). Consistent with changes in the pumping rate, the decline in locomotive activity of the worms was lessened by chicoric acid treatments; On day 12, chicoric acid-treated C. elegans moved 59\% and $86 \%$ faster than the control group, respectively (Fig. 4.3B).
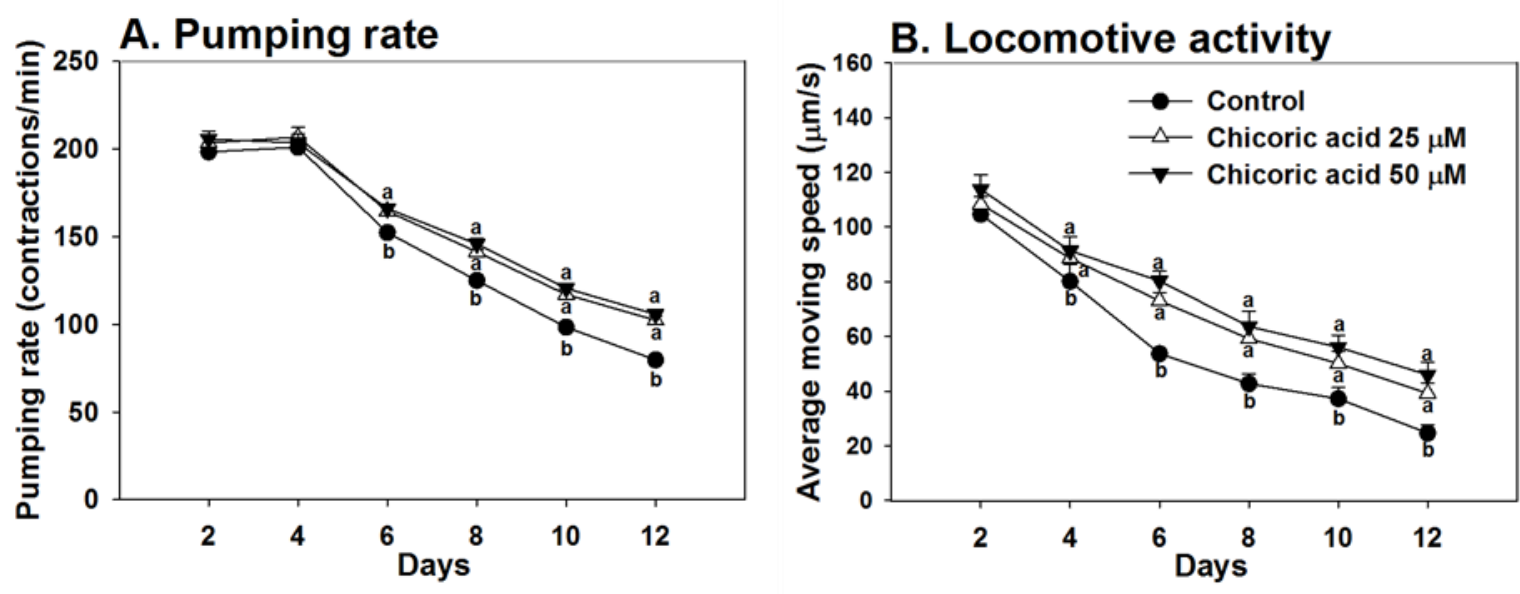

Figure 4.3 Chicoric acid ameliorated the age-related decline of movement and pumping rate. (A) Synchronized L1 C. elegans were treated with chicoric acid and pharyngeal 
pumping was counted every other day until day 12. (B) Synchronized L1 C. elegans were treated with chicoric acid and moving speed was recorded by WormLab tracking system. Then the average moving speed of each worm was calculated using WormLab Software. Values are means \pm S.E. $\mathrm{n}=12$ worms for pumping rate; $\mathrm{n}=140-180$ for worm speed. Values with different letters at each point in time are significantly different $(P<0.05)$.

Previous studies have reported that extended lifespan is closely associated with increased resistance towards oxidative stress $(93,94,96)$. Since chicoric acid is known to possess anti-oxidative properties, the intracellular ROS levels of C. elegans were determined after the treatment of chicoric acid. The intracellular ROS levels in the chicoric acid treatment groups were significantly reduced (14.6\% and $19.2 \%$ for 25 and $50 \mu \mathrm{M}$ chicoric acid with $P=0.0392$ and $P=0.0134$, respectively) compared with the control (Fig. 4.4).

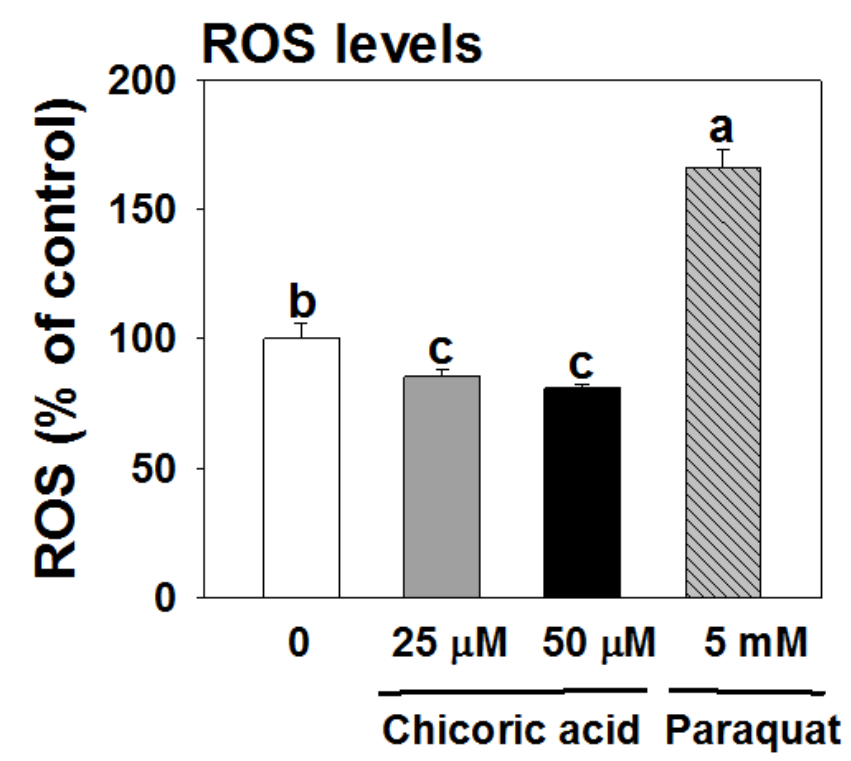

Figure 4.4 Effect of chicoric acid on the oxidative stress sensitivity of wild type N2 worms. Relative formation of reactive oxygen species (ROS) after $72 \mathrm{~h}$ of exposure to chicoric acid $(0,25$ or $50 \mu \mathrm{M})$. Paraquat $(5 \mathrm{mM})$ was used as the positive control. Values are means \pm S.E. ( $\mathrm{n}=9$; 60 worms/well). Values with different letters are significantly different $(P<0.05)$. 


\subsubsection{Extended lifespan by chicoric acid required AAK-2 and SKN-1}

Several studies reported that $a a k-2$ is an essential regulator in the aging of $C$. elegans through dietary restriction and/or stress resistance $(96,108-110)$. aak-2 encodes a homolog of the catalytic- $\alpha$ subunit of AMPK in C. elegans. The deletion of aak-2 shortens C. elegans lifespan, while the overexpression of aak-2 results in C. elegans lifespan extension $(108,109)$. Since chicoric acid has been found to upregulate AMPK in hepatocytes (52), we determined the role of AMPK homolog in chicoric acid's effects on lifespan extension using aak-2 loss-of function mutants. As shown in Table 4.1 and Fig $4.5 \mathrm{~A}$, the deletion of aak-2 abolished chicoric acid-mediated lifespan extension, indicating the requirement of aak-2 in C. elegans-lifespan extension by this compound.

In addition to $a a k-2$, it is known that the insulin/IGF-1 signaling pathway is involved in aging in nematodes $(111,112) . d a f-2$ encodes for the insulin-like growth factor 1 (IGF-1) receptor, which is an important regulator for aging in nematodes (113). Mutants deficient in $d a f-2$ exhibit longer lifespan due to an extended dauer stage (114). One of the downstream targets of $d a f-2$ is age-1, another gene mainly regulating the longevity of C. elegans (113). Furthermore, DAF-2 and AGE-1 have been reported to inhibit DAF-16, an important transcription factor that regulates C. elegans' lifespan (94). To determine whether chicoric acid extends lifespan through the insulin signaling pathway, we tested daf-2, age-1, and daf-16 null mutants. The daf-2 deficient and age-1 deficient mutants treated with 25 and $50 \mu \mathrm{M}$ chicoric acid displayed significantly extended median lifespan compared to the respective controls $(P<0.0001$ for both; Table 4.1 and Fig 4.5B-C). Similarly, the median lifespan of daf-16 mutants was significantly extended with the exposure of both 25 and $50 \mu \mathrm{M}$ chicoric acid compared to the control; 
14 days for the control (daf-16 mutants) vs. 18 and 20 days with chicoric acid at 25 and $50 \mu \mathrm{M}$, with $P=0.0012$ and $P=0.05$, respectively (Fig $4.5 \mathrm{D}$ ). These results indicate that chicoric acid may act independently of the insulin/IGF-1 signaling pathway in C. elegans (113).

In addition to AMPK and the insulin signaling pathway, several other molecular targets are known to regulate $C$. elegans lifespan, such as SKN-1 and EAT-2. SKN-1/Nrf2 transcription factor has been found to mediate C. elegans lifespan extension mainly through the oxidative stress response pathway $(115,116)$. From Table 4.1 and Fig 4.5E, skn-1 mutation abolished chicoric acid-induced lifespan extension, suggesting that $s k n-1$ is required for chicoric acid-mediated lifespan extension. Lastly, we tested eat-2 mutant, which has defects of pharyngeal pumping that leads to prolonged lifespan via dietary restriction pathway (117). As shown in Table 1 and Fig. 4.5F, chicoric acid treatments shortened the median lifespan of eat-2 deficient mutant, indicating the extended lifespan by chicoric acid might not occur through modulation of dietary intake. Since the observations that 25 and $50 \mu \mathrm{M}$ chicoric acid led to similar effects on the aging and lifespan of mutants, we used $25 \mu \mathrm{M}$ chicoric acid for the rest of the experiments. 
Table 4.1 Effect of chicoric acid on median lifespan of wild type and mutant C. elegans.

\begin{tabular}{|c|c|c|c|}
\hline Strain & $\begin{array}{c}\text { Chicoric acid } \\
\text { treatment }(\mu \mathrm{M})\end{array}$ & $\begin{array}{l}\text { Median lifespan }{ }^{\mathrm{a}} \\
\text { (days) }\end{array}$ & $\begin{array}{c}\text { Genetic } \\
\text { requirement }^{b}\end{array}$ \\
\hline \multirow[t]{3}{*}{ N2 } & Control & 19 & - \\
\hline & 25 & $23 * * *$ & \\
\hline & 50 & $22 * * *$ & \\
\hline \multirow[t]{3}{*}{ aak-2 (ok524) X } & Control & 12 & Yes \\
\hline & 25 & 12 & \\
\hline & 50 & 14 & \\
\hline \multirow[t]{3}{*}{ daf-2 (e1370) III } & Control & 32 & No \\
\hline & 25 & $36 * * *$ & \\
\hline & 50 & $38 * * *$ & \\
\hline \multirow[t]{3}{*}{ age-1 (hx546) II } & Control & 28 & No \\
\hline & 25 & $32 * * *$ & \\
\hline & 50 & $32 *$ & \\
\hline \multirow[t]{3}{*}{ daf-16 (mgDf50) I } & Control & 14 & No \\
\hline & 25 & $18 * *$ & \\
\hline & 50 & $20 * * *$ & \\
\hline \multirow[t]{3}{*}{ skn-1(zu135) IV } & Control & 16 & Yes \\
\hline & 25 & 18 & \\
\hline & 50 & 18 & \\
\hline \multirow[t]{3}{*}{ eat-2(ad1116) II } & Control & 32 & No \\
\hline & 25 & $24 * * *$ & \\
\hline & 50 & $26^{*}$ & \\
\hline
\end{tabular}

All strains were raised at $20^{\circ} \mathrm{C}$, except daf-2 (e1370) III, which was raised at $15^{\circ} \mathrm{C}$. Worms were treated with chicoric acid $(25$ or $50 \mu \mathrm{M})$ from the L4 stage (day 0 ).

Floxuridine (FUdR) was added with a final concentration of $120 \mu \mathrm{M}$. The survivals were recorded every other day until all the worms died. ${ }^{\text {a }}$ The median lifespan was referred to the time when survival rate dropped to $50 \%{ }^{*}, * *$, and $* * *$ mean significant difference compare with their respective control at $P<0.05, P<0.01$ and $P<0.001$, respectively (n= 180-266 worms/treatment). ${ }^{b}$ Significant differences were analyzed by Log-rank (MantelCox) tests. Genetic requirement was defined as "Yes" at $P<0.05$ or "No" at $P>0.05$. 

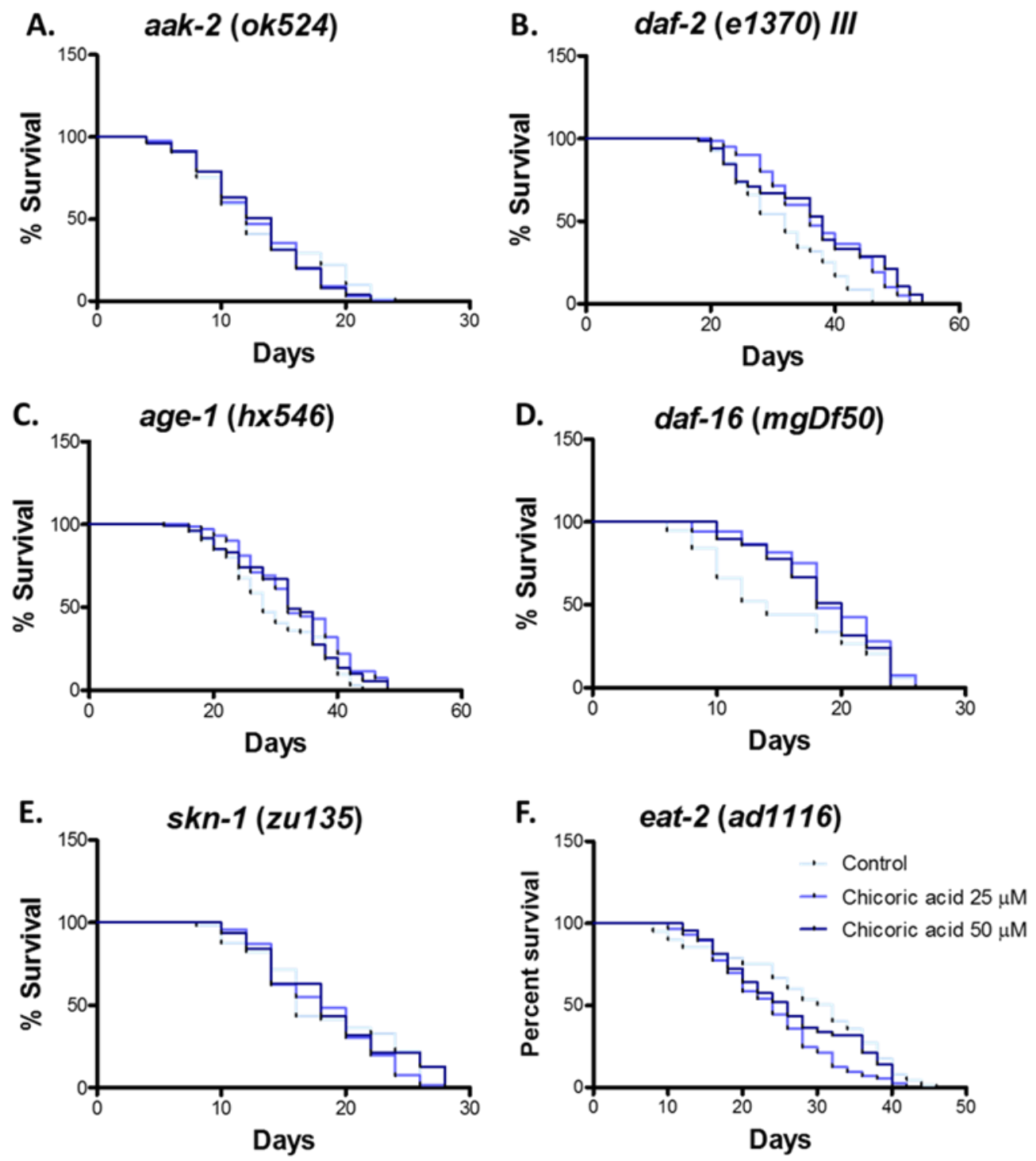

Figure 4.5. Survival of several mutant strains treated with chicoric acid. (A-F) Loss of function mutant strains in several genes related to lifespan regulation were treated with final concentrations of 0 (control), 25, or $50 \mu \mathrm{M}$ chicoric acid starting from L4 stage (day $0)$. The survivals were recorded every other day until all the mutants died $(\mathrm{n}=180-266$ worms/treatment). Log-rank (Mantel-Cox) test was used for statistical analysis.

\subsubsection{Chicoric acid might extend C. elegans lifespan via the regulation of AAK-2} and SKN-1. 
Since the loss of aak-2 and skn-1 abolished chicoric acid-mediated lifespan extension, we determined the role of aak-2 and skn-1 in chicoric acid's effect on lifespan extension. First, we found that chicoric acid upregulated the mRNA expression of aak-2 (Fig. 4.6A). Since it is known that AAK-2 can be regulated post-translationally $(97,118$, 119), we measured the role of chicoric acid in phosphorylation of AAK-2 and found that chicoric acid significantly increased the phospho-AAK-2 level $(P<0.001$, Fig. $4.6 \mathrm{~B}$ and C). We further determined the mRNA expression of a downstream target of AAK-2, $n h r$ 49, a homolog of peroxisome proliferator-activated receptor alpha (PPAR $\alpha)$ (120). As shown in Fig. 4.6A, chicoric acid treatment significantly increased expression of $n h r-49$ compared to the control $(P=0.0066)$.

Chicoric acid also significantly upregulated gene expression of skn-1 (Fig. 4.6A). Similar to AAK-2, SKN-1 can be regulated post-translationally, which can be measured by nuclear translocation of SKN-1 in C. elegans (121). Chicoric acid treatment significantly promoted SKN-1 translocation from the cytoplasm to the nuclei $(P=0.0041$, Fig 4.6D), suggesting potential post-translational regulation of SKN-1 by chicoric acid. Next, we further tested two SKN-1 target genes, gsr-1 (glutathione reductase) and $p b s-5$ (proteasome beta subunit) (122-124), which were increased by chicoric acid treatment - a 1.7-fold and 2.4-fold increase over the control, with $P=0.0259$ and $P=0.0112$, respectively (Fig. 5A). 
A. Gene expression in wild type worms

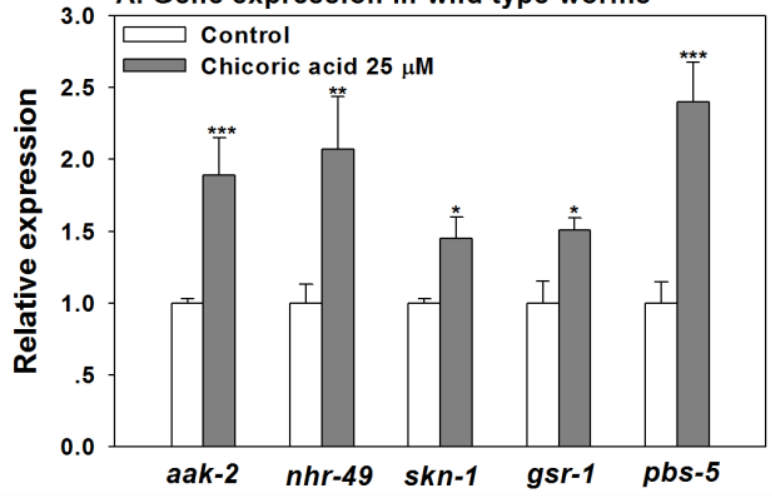

B.
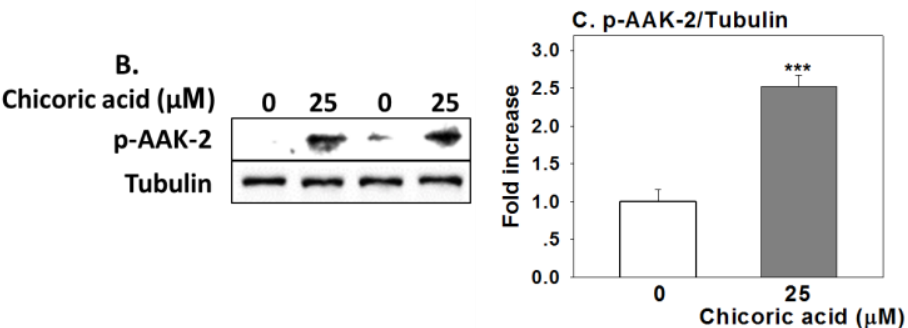

D.

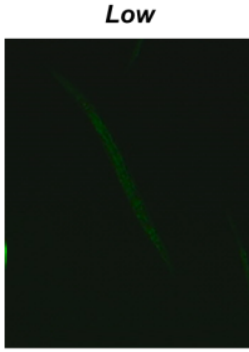

Medium

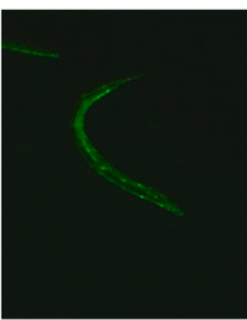

High

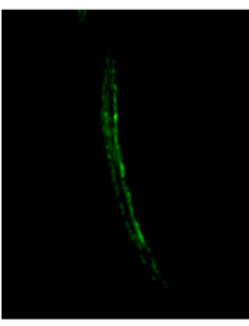

$500 \mu \mathrm{m}$

E. SKN-1 nuclear translocation

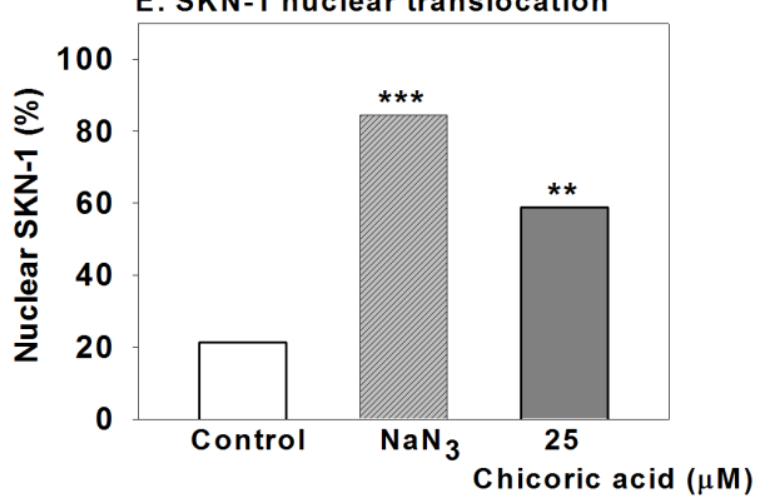

Figure 4.6 Chicoric acid might extend C. elegans lifespan via the regulation of AAK-2 and SKN-1. Synchronized L1 worms were treated with 0 or $25 \mu \mathrm{M}$ chicoric acid for $48 \mathrm{~h}$ (A). The mRNA expression of aak-2, nhr-49, skn-1,gsr-1, and $p b s-5$ were determined by real-time PCR. ama-1 was used as an internal control. (B) Phospho-AAK-2 and tubulin were determined by immunoblotting. (C) p-AAK-2/Tubulin, phospho-AAK-2/Tubulin. 
Values are means \pm S.E. $(\mathrm{n}=3),{ }^{*} P<0.05, * * P<0.01$, and $* * * P<0.001$. (D) Intracellular localization of SKN-1::GFP. The nuclear translocation patterns of SKN-1::GFP were identified as 'low', 'medium' and 'high'. (E) Nuclear translocation of SKN-1 in ldIs 7; worms treated with chicoric acid were analyzed using the chi square test, and therefore do not show standard error bars (control, $\mathrm{n}=94$; NaN3, $\mathrm{n}=82$; chicoric acid $25 \mu \mathrm{M}, \mathrm{n}=$ 107). A NaN3 (2\%) treated group was used as the positive control. ** and *** mean significant difference compared with the control at $P<0.01$ and $P<0.001$, respectively.

\subsubsection{Chicoric acid enhanced oxidative stress resistance in wild type $\mathrm{N} 2$ worms but}

\section{not aak-2 mutants}

As $a a k-2$ has been reported to regulate oxidative stress resistance in nematodes (119), we then evaluated the influence of chicoric acid on oxidative stress responses of wild type worms and aak-2 mutants. After 2, 4, and 6 days of paraquat exposure, the survival of chicoric acid-treated wild type worms was consistently improved over that of the control $(12.2 \%, 12.8 \%$, and $20.8 \%$ improvement for $25 \mu \mathrm{M}$ chicoric acid with $P<0.001, P=0.002$, and $P<0.001$, respectively, Fig 4.7A). As shown in Fig 4.7B, the beneficial effect of chicoric acid against oxidative stress was abolished in aak-2 mutants, suggesting an essential role of AAK-2 in chicoric acid-enhanced oxidative stress resistance in C. elegans. 

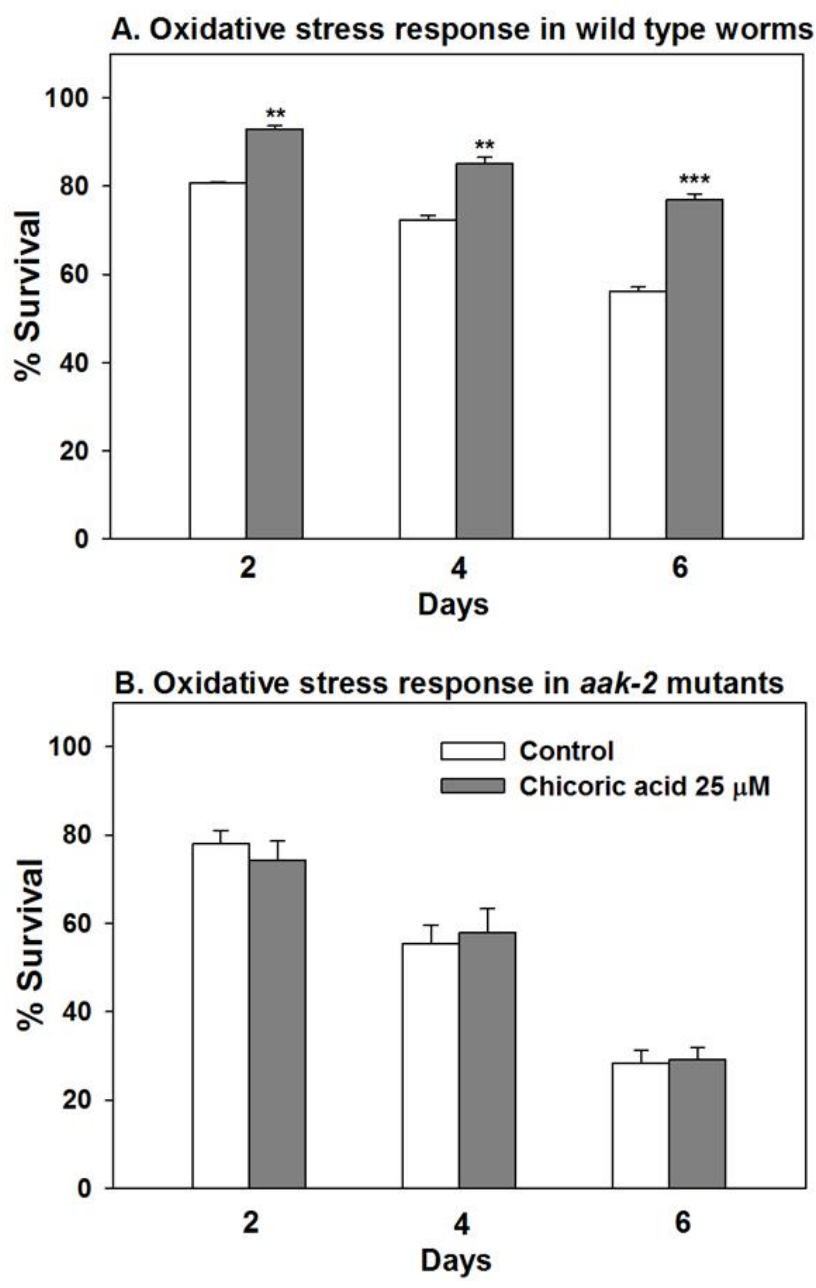

Figure 4.7 Chicoric acid enhanced the stress resistance of wild type worms but not aak-2 mutants. Wild type worms and aak-2 mutants (day 1 adults) were exposed to $5 \mathrm{mM}$ paraquat with or without chicoric acid for 2, 4, and 6 days and survivals were counted. Values are means \pm S.E. $(\mathrm{n}=3 ; 120-145$ worms/treatment $), * * P<0.01$ and $* * * P<0.001$.

\subsubsection{Discussion}

In the current study, we found that chicoric acid at 25 and $50 \mu \mathrm{M}$ significantly extended the lifespan of $C$. elegans. These findings are consistent with a previous study showing that chicoric acid at $25 \mu \mathrm{M}$ or higher concentrations extended $C$. elegans lifespan (7). Here, we further investigated that chicoric acid-induced lifespan extension was accompanied by the prevention of age-associated declines of pumping rate and 
locomotor activity as well as reduced oxidative stress sensitivity without influencing other physiological functions. The effects of chicoric acid was determined to be mediated in part by cellular energy sensor, AAK-2, and oxidative stress responsive transcription factor, SKN-1, but not via the insulin signaling pathway or dietary restriction.

C. elegans lifespan extension is closely associated with enhanced stress resistance, including oxidative stress (125). Increased oxidative stress, resulting from the excess production of free radicals, typically ROS, accelerates the aging of C. elegans (70). Here, we found that chicoric acid reduced ROS level in vivo and increased survivals under an oxidative stress condition, which is consistent with previous reports that chicoric acid reduced ROS and enhanced oxidative stress resistance in RGC-5 rat retinal ganglion cells and SH-SY5Y human neuroblastoma $(67,75)$. Antioxidative compounds, including chicoric acid, may directly act as ROS scavenger and/or indirectly activate stress-related signaling pathways, although based on the current results, it is not clear how chicoric acid elicits its effects on ROS.

The aak-2 gene encodes an isomer of the AMPKa in C. elegans (108). A few studies have reported that $a a k-2$ is intimately involved in the control of C. elegans lifespan through the regulation of dietary restriction and/or stress resistance $(96,108-$ 110). Based on the significant effect by chicoric acid on eat-2 mutants, we determined that chicoric acid elicits its protective effects via stress resistance. In particular, Lee et al. (119) reported that aak-2 mutations are hypersensitive to paraquat-induced oxidative stress, suggesting an important role of aak-2 to C. elegans oxidative stress responses. This is supported by the current observation that aak-2 deficiency completely abolished 
the chicoric acid-mediated lifespan extension and survival during the oxidative stress conditions in C. elegans (Table 4.1 and Fig.4.5). The $n h r-49$ is one of the downstream genes of aak-2 (120), and it was suggested that NHR-49 may participate in oxidative stress responses and prevent ROS-induced toxicity in C. elegans (121). In fact, MorenoArriola et al. suggested that phospho-AAK-2 can regulate oxidative metabolism through increased transcriptional activity of $n h r-49$ (126). Thus, chicoric acid may extend lifespan in part via a mechanism dependent upon aak-2 and its target, $n h r-49$. However, since we were not able to determine the effects of chicoric acid on AAK-2 due to lack of available antibodies for AAK-2 of C. elegans, it is not conclusive that chicoric acid upregulated AAK-2 transcriptionally and/or post-translationally with the current results only.

$s k n-1$ is also involved in chicoric acid-induced lifespan extension in C. elegans. SKN-1 starts regulating C. elegans lifespan as early as the postembryonic stage by activating cellular defense responses to oxidative stress (127). This was further supported by the fact that loss of function mutants in $s k n-1$ are sensitive to oxidative stress and the transgene of SKN-1::GFP rescues their embryonic developmental defect (115). It was reported that nuclear translocation of SKN-1 results in the higher cellular oxidative stress resistance in C. elegans (121). The current study showed that chicoric acid induced translocation of SKN-1 from the cytoplasm to nuclei with minimum enhancement of $s k n$ 1 expression. This may suggest that chicoric acid's effect on post-translational regulation of SKN-1 contributes significantly to the antioxidation and lifespan benefits of chicoric acid in C. elegans. This was further confirmed by the observation of increased expression with chicoric acid treatment of two downstream genes of SKN-1, glutathione reductase $(g s r-1)$ and proteasome subunit ( $p b s-5)$, which are essential for oxidative stress tolerance 
in C. elegans (122). However, it is not clear if chicoric acid regulates SKN-1 directly or by regulating upstream regulators of SKN-1. In fact, AAK-2 was suggested to be the upstream of SKN-1 for metformin-mediated lifespan extension in a previous publication (96). Moreover, another study suggested that glycogen synthase kinase-3 (GSK-3) is the upstream of SKN-1 contributing to the increased resistance of oxidative stress in $C$. elegans (128). Thus, future experiments are needed to determine the target of chicoric acid on enhanced oxidative stress resistance.

Moreover, both AAK-2 and SKN-1 have been reported to regulate mitochondrial biogenesis against oxidative stress and contribute to longevity in C. elegans, suggesting the possible effect of chicoric acid on mitochondrial function $(98,129)$. One study reported that chicoric acid promotes mitochondrial biogenesis by increasing citrate synthase activity and the deacetylation of peroxisome proliferator-activated receptor- $\gamma$ coactivator, which is directly activated by AMPK $\alpha$ (7). Thus, we cannot exclude the possibility that chicoric acid extends lifespan via a mitochondrial biogenesis-mediated mechanism.

Chicoric acid is metabolized to 2-caffeoyl-L-tartaric acid (cafraric acid) and caffeic acid by rat liver microsomes (76). A recent study reported that chicoric acid has low absorption in rats, with peak plasma concentration at $3.44 \pm 0.53 \mu \mathrm{M}$ after $4 \mathrm{~h}$ of oral administration of chicoric acid at $50 \mathrm{mg} / \mathrm{kg}$ body weight (91). Thus, the $25 \mu \mathrm{M}$ of chicoric acid used in the current study might be difficult to achieve in animals and humans. Moreover, currently the comparability of chicoric plasma concentration between 
C. elegans and other in vivo models is not known; thus, it is important to determine the biological relevance of chicoric acid in humans.

In conclusion, chicoric acid significantly extended the lifespan of $C$. elegans in part through regulation of $a a k-2$ and $s k n-1$. Because of the high conservation of these two genes between nematodes and mammals, these findings indicate that chicoric acid may have potential for promoting healthy aging as well as combatting age-related diseases in humans.

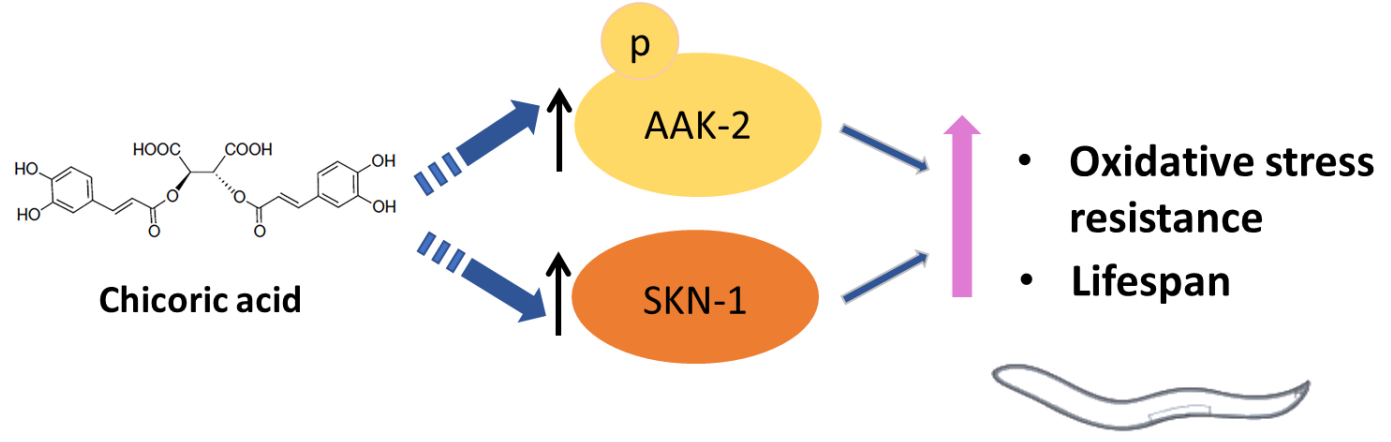

Fig. 4.8 The graphic contents. Chicoric acid significantly extended the lifespan of $C$. elegans and increase the oxidative stress resistance in part through regulation of aak-2 and $s k n-1$. 


\subsection{Effect of chicoric acid on glucose uptake and the underlying mechanism}

\subsubsection{Introduction}

Type 2 diabetes mellitus (T2DM) has been recognized as a major public health challenge throughout the world in recent decades $(8,9)$. Impaired glucose uptake in muscle tissues is the primary associated with hyperglycemia in T2DM (10). Upon insulin signaling, protein kinase B, also known as Akt, is activated by phosphorylation that leads to translocation of glucose transporter 4 (GLUT4) to the plasma membrane, which subsequently promotes glucose uptake $(126,130-132)$. In addition to insulin signaling, several studies indicated that AMP-activated protein kinase $\alpha$ (AMPK $\alpha)$, which is known to regulate energy homeostasis $(133,134)$, also activates Akt, independent of insulin, and results in increased glucose uptake $(135,136)$.

Accumulating evidence suggests that many natural bioactive components have great potential to be used for the prevention or amelioration of T2DM $(126,137)$. Among natural bioactives, chicoric acid has been recently reported to have potential anti-diabetic effects $(11,51,73)$. It is a naturally occurring dicaffeoyl ester often found in chicory plants and basil (Fig. 1A) $(138,139)$. One study reported that chicoric acid promoted glucose uptake in L6 muscle cells (11). More recently, another study revealed that chicoric acid regulated glucose homeostasis, stimulated the AMPK $\alpha$ pathway, and reversed insulin resistance in HepG2 cells (52). However, it is still not clear whether chicoric acid enhances glucose uptake in muscle cells via an AMPK $\alpha$-mediated pathway. A better understanding of chicoric acid's impact in promoting glucose uptake in muscle cells would be significant given that muscle is responsible for approximately $75 \%$ of glucose disposal in the body (140). We used C2C12 myotubes, derived from murine 
skeletal muscle cells and a C57BL/6J mice model to determine the role of chicoric acid on glucose homeostasis and its underlying mechanisms $(141,142)$.

\subsubsection{Materials and methods}

\subsubsection{Materials}

Chicoric acid ( $\geq 98 \%$ ) was obtained from Santa Cruz Biotechnology, Inc. (Santa Cruz, CA, USA). C2C12 murine skeletal myoblasts were from American Type Cell Collection (Manassas, VA, USA). Bovine serum albumin, recombinant human insulin, and a penicillin/streptomycin mixture were purchased from Thermo Fisher (Waltham, MA, USA). Dulbecco's Modified Eagle's Medium (DMEM), Kaighn's Modification of Ham's F-12 (F-12K), fetal bovine serum (FBS), dimethyl sulfoxide (DMSO), 3-(4,5dimethylthiazol-2-yl)-2,5-diphenyltetrazolium bromide (MTT, purity $\geq 93 \%$ ), and horse serum (HS) were obtained from Sigma-Aldrich (St. Louis, MO, USA). Dorsomorphin dihydrochloride (purity $\geq 98 \%$ ), also named Compound C, was purchased from MedChem Express (Monmouth Junction, NJ, USA). The protein content was determined using protein DC assay kits from Bio-Rad Co. (Hercules, CA, USA). Rabbit antibodies for phospho-Akt (Ser473), Akt (total), phospho-AMPKa, AMPKa (total), phospho-acetylCoA carboxylase (ACC) (Ser79), ACC (total), mouse antibody for GLUT4, goat antirabbit IgG-horseradish peroxidase (HRP), and goat anti-mouse IgG-HRP were obtained from Cell Signaling Technology (Danvers, MA, USA). Rabbit antibodies for $\beta$-actin and $\mathrm{Na}^{+} / \mathrm{K}^{+}$-ATPase were purchased from Santa Cruz Biotechnology, Inc (Dallas, TX, USA).

\subsubsection{C2C12 culture}


$\mathrm{C} 2 \mathrm{C} 12$ cells were cultured in a $5 \% \mathrm{CO}_{2}$ atmosphere at $37^{\circ} \mathrm{C}$. Myoblasts were cultured and differentiated into multinucleated myotubes based on previous studies (143). After 6 days of differentiation, myotubes were treated with chicoric acid (12.5, 25, and 50 $\mu \mathrm{M})$ for $24 \mathrm{~h}$. For insulin treatment, $100 \mathrm{nM}$ insulin was added into media for $30 \mathrm{~min}$ before the harvest of cells. A previous study reported that $20 \mu \mathrm{M}$ Compound $\mathrm{C}$ inhibited the activity of AMPK $\alpha$ in primary hepatocytes (144). In the current model, $10 \mu \mathrm{M}$ Compound $\mathrm{C}$ was able to inhibit $\mathrm{AMPK} \alpha$ phosphorylation in $\mathrm{C} 2 \mathrm{C} 12$ cells. Thus, $10 \mu \mathrm{M}$ Compound C was used in this study. An MTT-based cell viability test was used to determine the cytotoxicity of chicoric acid (145). Briefly, C2C12 myoblasts were seeded in 96-well plates at a density of $1 \times 10^{6}$ cells $/ \mathrm{mL}$. Cells were treated with $12.5,25$, and 50 $\mu \mathrm{M}$ of chicoric acid for $24 \mathrm{~h}$. The medium was then replaced with $5 \mathrm{mg} / \mathrm{mL}$ MTT in DMEM (without phenol red) for $4 \mathrm{~h}$ at $37^{\circ} \mathrm{C}$. After incubation, cells were washed thrice with phosphate-buffered saline (PBS) and formazan crystals dissolved in DMSO were measured with microplate reader SpectraMax i3 (Sunnyvale, CA, USA) at $570 \mathrm{~nm}$. Concentrations of chicoric acid tested $(0-50 \mu \mathrm{M})$ were not toxic to $\mathrm{C} 2 \mathrm{C} 12$ cells as shown in Fig. 4.9A.

\subsubsection{Glucose uptake assay}

For glucose uptake measurement, 2-(N-(7-nitrobenz-2-oxa-1,3-diazol-4yl)amino)-2-deoxyglucose (2-NBDG), a fluorescent derivative of glucose, was used as described previously (143). Myoblasts were seeded in the 24-well plates. After 6 days of differentiation, myotubes were treated with various doses of chicoric acid in F-12K (5.5 mM glucose) serum free media for different time periods as shown the Figure legends. 
C2C12 myotubes were then treated with and without $100 \mathrm{nM}$ insulin for $30 \mathrm{~min}$ in a Krebs-Ringer buffer that was prepared as published previously (146). Insulin (30 min) stimulation was used to activate insulin signaling in $\mathrm{C} 2 \mathrm{C} 12$ myotubes as previously described $(147,148)$. After insulin stimulation, the 2-NBDG solution was added to each well at a final concentration of $200 \mu \mathrm{M}$, and the cells were incubated at $37^{\circ} \mathrm{C}$ for $30 \mathrm{~min}$. Cells were then rinsed thrice with ice cold PBS and the fluorescence was immediately measured using the SpectraMax i3 microplate reader at excitation/emission 465/540 nm. The cell lysate was prepared by adding $40 \mu 10.1 \mathrm{M} \mathrm{NaOH}$ per well. The protein content of each well was determined by protein DC assay kits. The fluorescence was normalized by protein content.

\subsubsection{Cell membrane protein extraction}

Integral membrane proteins and membrane-related proteins from $\mathrm{C} 2 \mathrm{C} 12$ myotubes were prepared using a Mem-PER Plus Membrane Protein Extraction Kit from Thermo Fisher Scientific (Waltham, MA, USA) according to the manufacturer's instructions. GLUT4 translocated to membrane and total GLUT4 were determined by immunoblotting.

\subsubsection{Transfection of shRNA-AMPKa}

Lentivirus-mediated short hairpin RNA (shRNA) targeting of the mouse AMPK $\alpha 1$ gene (shAMPK $\alpha$ ) was established by Shanghai R\&S Biotechnology Co. Ltd (Shanghai, China). Detailed information is provided in supplementary data (Supplementary Table S1 and Figure S2). The effective target sequence 
ACGAGTTGACCGGACATAAAA in the mouse AMPKa1 gene (GeneID:

NM_001013367.3) was selected, and a scrambled sequence:

GAAATGTACTGCGCGTGGAGACGTTTTGGCCACTGACTGACGTCTCCACGCA

GTACATTT that had no significant homology to any mouse gene sequences was used as a negative control (shControl). Transfection of shRNA was performed based on the manufacturer's instructions. A virus counted with approximately 100 multiplicity of infection (MOI) was used to infect one well of cells in a 6-well plate for $12 \mathrm{~h}$ in a serumfree medium with polybrene $(8 \mu \mathrm{g} / \mathrm{mL})$, and the cell culture medium was then changed to a normal medium containing 10\% FBS. After $48 \mathrm{~h}$ incubation, cells were treated with chicoric acid $(0$ or $25 \mu \mathrm{M})$ for $24 \mathrm{~h}$. AMPK $\alpha$ protein levels were measured by Western blotting.

\subsubsection{Western blotting}

C2C12 cell lysate was prepared for immunoblotting as described previously (107, 148). Protein concentrations were determined by the protein DC assay kits. $\beta$-Actin was used as an internal control. Horseradish peroxidase-conjugated goat anti-rabbit IgG was used as the secondary antibody. Protein blots were pictured by the Image Station 4000MM (Carestream Health, New Haven, CT, USA) with a Clarity Western ECL Substrate Kit (Bio-Rad Co.). Images were quantified with ImageJ software (version 1.49, National Institutes of Health, MD, USA).

\subsubsection{AMP/ATP assay}

The measurement of AMP and ATP from $\mathrm{C} 2 \mathrm{C} 12$ myotubes was conducted using 
a mouse AMP/ATP ELISA kit (Shanghai Fan Ke Biotechnology Co., Ltd., Shanghai, China). Briefly, C2C12 cells were treated with chicoric acid $(25 \mu \mathrm{M})$ for $24 \mathrm{~h}$, followed by washing with cold PBS twice at $4^{\circ} \mathrm{C}$. Then cells were diluted by PBS to reach the final concentration $1 \times 10^{7}$ cells $/ \mathrm{ml}$. The diluted cells were lysed with a lysis buffer according to the manufacturer's instructions. The supernatant was obtained by centrifugation for 20 min at the speed of $3000 \mathrm{rpm}$. Then the supernatant of cell lysate was taken to detect the levels of AMP and ATP following the protocol of the ELISA kit. The absorbance of each sample was read by the Multiskan FC microplate reader (Thermo Fisher Scientific, Waltham, MA, USA) at 450nm after adding a stop solution for $15 \mathrm{~min}$.

\subsubsection{Glucose tolerance test (GTT)}

Male C57BL/6J mice (8 week) were obtained from the Laboratory Animal Research Center of Jiangsu University (Jiangsu, China) and maintained on a $12 \mathrm{~h}$ light/dark cycle with free access to food and water for 2 weeks. Institutional guidelines (serial No. UJS-LAER-2018042301) for animal care and use were followed, and the animal protocol was approved by the animal ethics committee of Jiangsu University. After adaptation, the mice were divided into two groups (6-8 mice/group). For the treatment groups, mice received a daily oral administration of $5 \mathrm{mg} / \mathrm{kg} \mathrm{BW}$ chicoric acid for 5 days, based on a previous publication (59). For the control group, mice will be administrated with saline solution orally. Mice body weight will be measured before glucose tolerance test (GTT). On the fifth day, all mice will be fasted for $12 \mathrm{~h}$ following the first measurement of blood glucose from the tail vein by a blood glucose meter $(0 \mathrm{~min}$, ACCU-CHEK, Jiangsu, China). Then, all mice will be subjected to an intraperitoneal 
GTT (2 g glucose/kg BW). The blood glucose levels will be further monitored at 15, 30, 60, and $120 \mathrm{~min}$. The areas under the curves (AUCs) were calculated with Sigma Plot (version 11.0, Systat Software, Inc, San Jose, CA, USA).

\subsubsection{Statistical analysis}

SAS software (version 9.3, SAS Institute Inc., Cary, NC, USA) was used for statistical analysis. One-way analysis of variance (ANOVA) was used for all data except for Fig. 4.9B, Fig. 4.10A, Fig. 4.11B, Fig. 4.13, and Fig. 4.14, where two-way ANOVA with least squares means statement was used. When there were no interactions between chicoric acid and another factor (insulin or time), no letter was used in the figures. If there were significant interactions between chicoric acid and another factor (Compound C or shAMPK), letters were used in the figures to present the differences between each group. The Tukey-Kramer method was applied for the multiple comparisons among the groups. A difference was considered significant at $P<0.05$.

\subsubsection{Results}

\subsubsection{Chicoric acid promoted glucose uptake}

When overall glucose uptake was measured by using 2-NBDG from C2C12 myotubes, there were significant effects of insulin $(P=0.0006)$ and chicoric acid $(P<$ 0.0001) without any significant interaction between insulin and chicoric acid (Fig. 4.9A). Glucose uptake in insulin-stimulated groups was 20\%-25\% higher than in non-insulinstimulated groups. This is consistent with previous reports that less than $30 \%$ increase of glucose uptake after insulin stimulation with 2-NBDG $(149,150)$. The exposure of 
$\mathrm{C} 2 \mathrm{C} 12$ cells to chicoric acid at the concentrations of 25 and $50 \mu \mathrm{M}$ resulted in significant increases in glucose uptake regardless of insulin stimulation compared to the controls $(P=$ 0.001 and $P=0.009$, respectively, Fig. 4.9B). The effect of increased glucose uptake by chicoric acid $(25 \mu \mathrm{M})$ peaked after a $24 \mathrm{~h}$ treatment, and was significantly $36 \%$ greater than the control group $(P<0.0001$; Fig. $4.9 \mathrm{C})$; thus, a $24 \mathrm{~h}$ chicoric acid treatment was used for the subsequent experiments. 

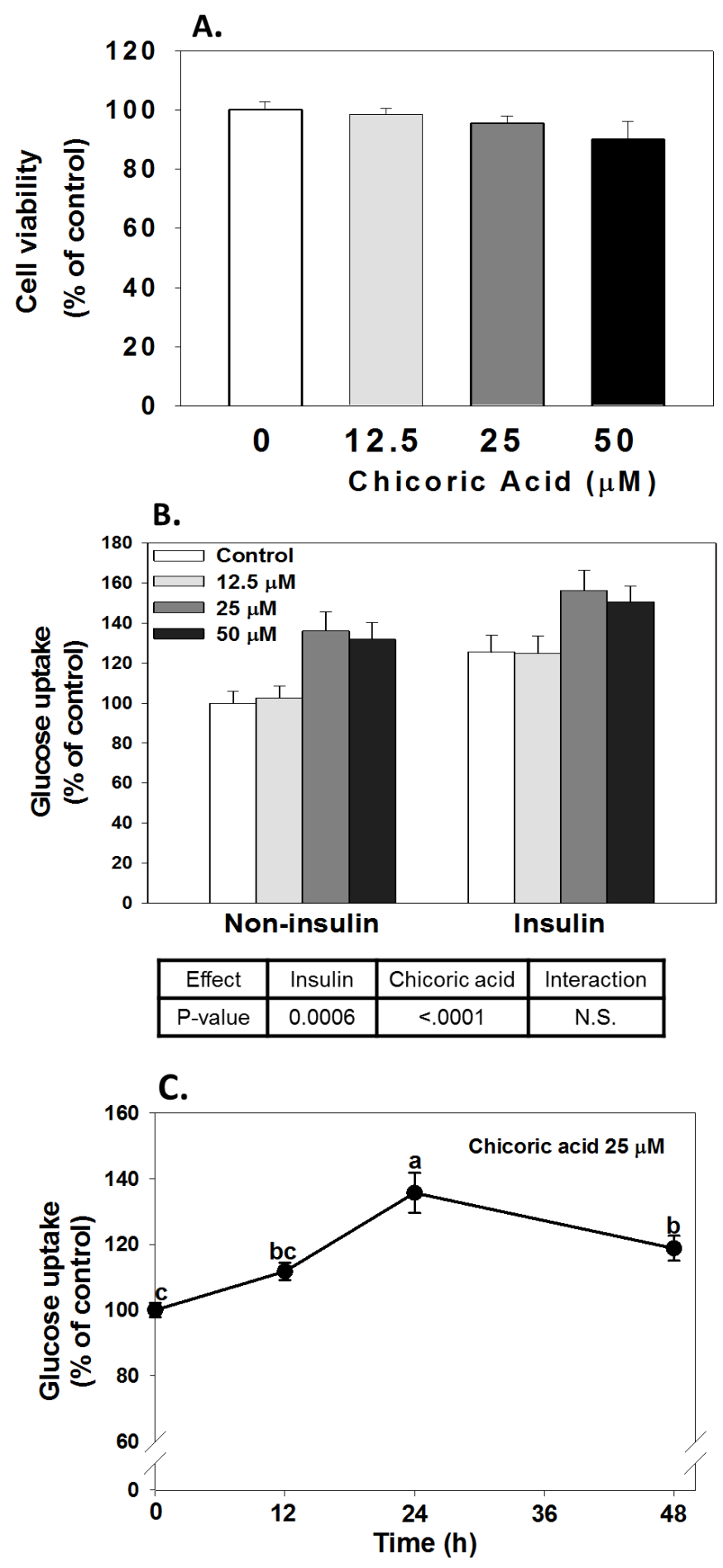

Figure 4.9 Chicoric acid promoted glucose uptake in $\mathrm{C} 2 \mathrm{C} 12$ myotubes. (A) Concentrations of chicoric acid tested $(0-50 \mu \mathrm{M})$ were not toxic to $\mathrm{C} 2 \mathrm{C} 12$ cells. (B) Glucose uptake was measured from myotubes that were treated with chicoric acid (12.5, 25 or $50 \mu \mathrm{M}$ ) for $24 \mathrm{~h}$, followed by a 30-min treatment with or without $100 \mathrm{nM}$ of insulin. (C) Myotubes were treated with $25 \mu \mathrm{M}$ chicoric acid for $12 \mathrm{~h}, 24 \mathrm{~h}$ or $48 \mathrm{~h}$ in F-12K (5.5 
$\mathrm{mM}$ glucose) serum free media, respectively. Since glucose uptake was induced by chicoric acid independent of insulin, no insulin stimulation was conducted in this test. After washing thrice, 2-NBDG solution was added to each well at a final concentration of $200 \mu \mathrm{M}$, and the cells were incubated at $37^{\circ} \mathrm{C}$ for $30 \mathrm{~min}$. Cells were then rinsed thrice with ice cold PBS and the fluorescence was immediately measured using the SpectraMax i3 microplate reader at excitation/emission $465 / 540 \mathrm{~nm}$. Numbers are mean \pm S.E. $(\mathrm{n}=5)$. Means with different letters are significantly different at $P<0.05$.

We also conducted in vivo glucose tolerance tests (GTT) to determine if chicoric acid supplementation would have the similar results. There were significant effects in GTT for time $(P<0.0001)$ and chicoric acid $(P<0.0001)$ without any significant interaction between time and chicoric acid (Fig. 4.10A). Chicoric acid treatment (5 mg/kg BW for 5 days) led to a $14.5 \%$ reduction of the overall blood glucose level compared to the control $(P<0.0001 ;$ Fig. 2A). The areas under the curves (AUCs) in GTT further confirms that chicoric acid treatments improved glucose tolerance in mice $(P=0.0065$, Fig. 4.10B). 


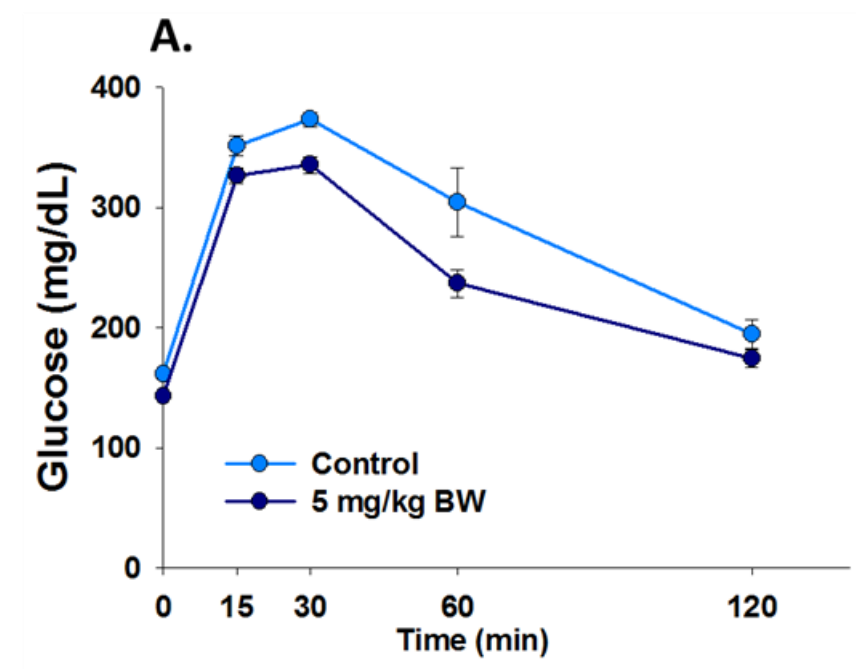

\begin{tabular}{|c|c|c|c|}
\hline Effect & Time & Chicoric acid & Interaction \\
\hline P-value & $<.0001$ & $<.0001$ & N.S. \\
\hline
\end{tabular}

B.

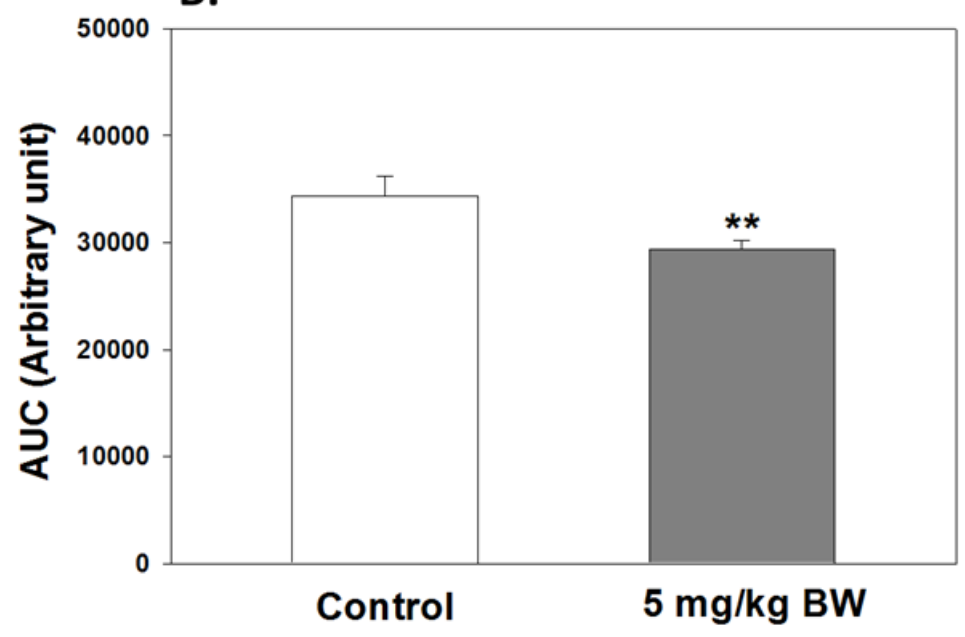

Fig. 4.10 Effects of chicoric acid on glucose tolerance test (GTT). Mice were administrated with ( 0 and $5 \mathrm{mg} / \mathrm{kg}$ BW/day) chicoric acid orally for 5 days. (A) Blood was collected from the tail vein, and glucose levels were measured at $0 \mathrm{~min}$, then the glucose solution ( $2 \mathrm{~g} / \mathrm{kg} \mathrm{BW}$ ) was administered by intraperitoneal injection and blood glucose level were determined at 15, 30,60 and $120 \mathrm{~min}$. (B) Area under the curve (AUC). Values are means \pm S.E. $(\mathrm{n}=6), * * P<0.01$. Means with different letters are significantly different at $P<0.05$. 


\subsubsection{Effects of chicoric acid on Akt activation and GLUT4 translocation in C2C12 myotubes}

It is known that the activation of Akt (phosphorylated-Akt, p-Akt) and GLUT4 translocation to the plasma membrane directly regulate glucose uptake in muscle cells $(9$, 131). Thus, we examined the effects of chicoric acid on the phosphorylation of Akt and GLUT4 translocation from C2C12 myotubes. Fig. 4.11A and 4.11B show that the ratio of $\mathrm{p}$-Akt/Akt (as an indicator of activation of Akt) was significantly increased by insulin $(P<0.0001)$ and chicoric acid $(P=0.0003)$, without any interaction between insulin and chicoric acid. These results are consistent with the results in Fig. 4.9A that chicoric acid promoted glucose uptake in an insulin-independent manner. From these results, we selected $25 \mu \mathrm{M}$ chicoric acid to determine its effect on GLUT4 translocation since this concentration had the highest activation of Akt, a $132 \%$ increase over the control in this model $(P<0.0001$; Fig. 4.11A). As shown in Fig 4.11C-E, the treatment of $25 \mu \mathrm{M}$ chicoric acid resulted in a $28 \%$ increase in GLUT4 translocation to the cell membrane compared to the control $(P=0.0086)$ without altering total GLUT4 expression. These results suggest that chicoric acid activated Akt independently of insulin and promoted GLUT4 translocation to the plasma membrane. 

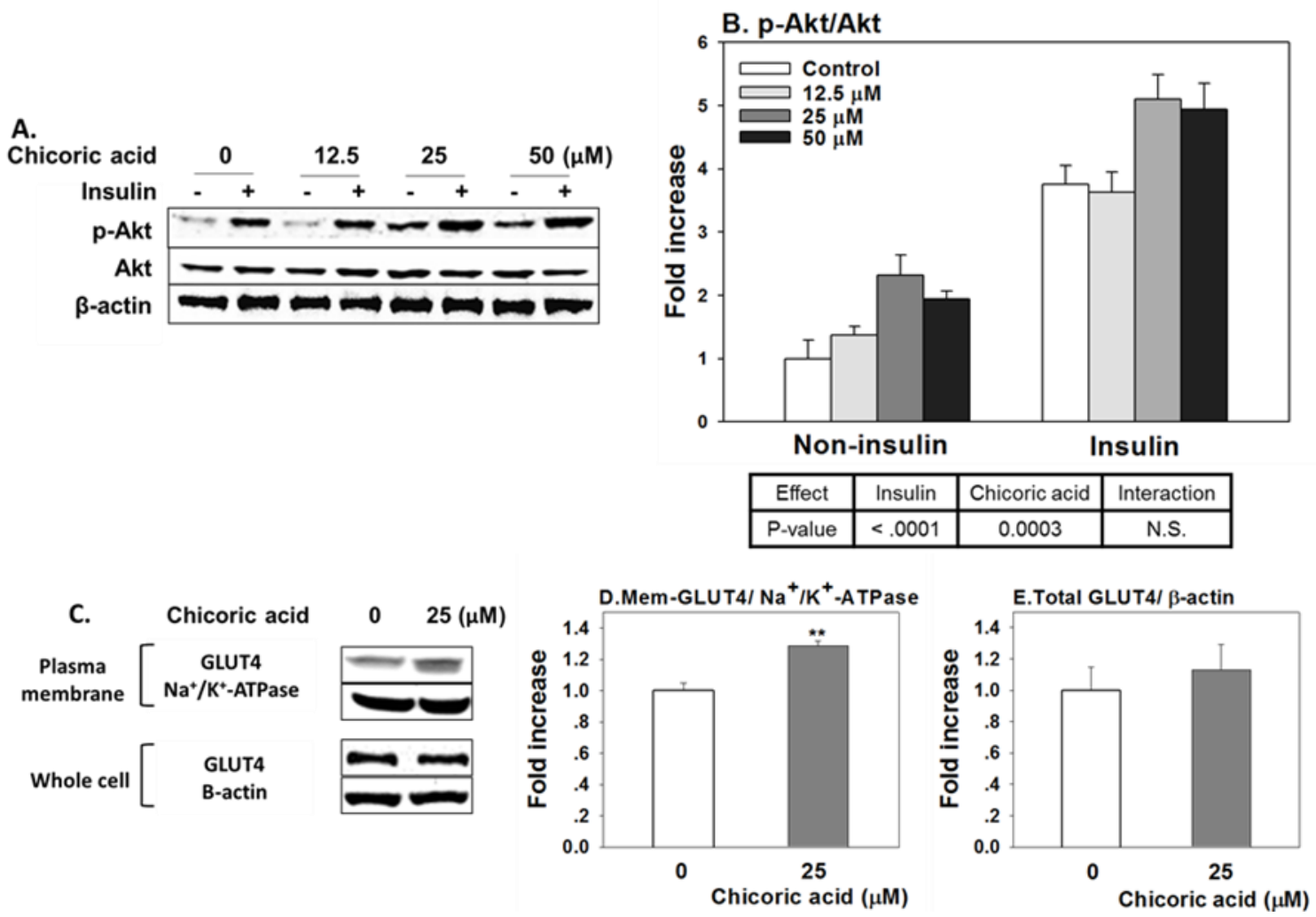

Figure 4.11 Activation of Akt and translocation of glucose transporter 4 (GLUT4) by chicoric acid in $\mathrm{C} 2 \mathrm{C} 12$ myotubes. (A) Myotubes were treated with the indicated concentrations of chicoric acid for $24 \mathrm{~h}$ and/or $100 \mathrm{nM}$ insulin for $30 \mathrm{~min}$ in F-12K serum-free medium. Then phosphorylation of Akt and total Akt was determined by immunoblotting. (B) p-Akt/Akt, phosphorylated protein kinase B/protein kinase B. (C) Chicoric acid induced translocation of GLUT4. C2C12 myotubes were treated with 25 $\mu \mathrm{M}$ of chicoric acid for $24 \mathrm{~h}$. Then the protein of plasma membrane and whole cells were extracted to immunoblotting. (D) Mem-GLUT4/ $\mathrm{Na}^{+} / \mathrm{K}^{+}$-ATPase, GLUT4 on cell membrane/internal reference on cell membrane. (E) Total GLUT4/ $\beta$-actin, GLUT4 in whole cells/internal reference in whole cells. Numbers are mean \pm S.E. $(n=3), * * P<0.01$. Means with different letters are significantly different at $P<0.05$.

\subsubsection{Effects of chicoric acid on the AMPKa signaling pathway in C2C12}

\section{myotubes}

Based on previous reports that chicoric acid promoted AMPK $\alpha$ activation in L6 myocytes (73), and AMPK $\alpha$ can activate $\operatorname{Akt}(135,136)$, we determine if the effect of chicoric acid (12.5 and $25 \mu \mathrm{M})$ on Akt was dependent upon AMPKa (Fig. 4.12). First, 
we have confirmed that chicoric acid $(25 \mu \mathrm{M})$ activated AMPK $\alpha$ (the ratio of $\mathrm{p}$ AMPK $\alpha / \mathrm{AMPK} \alpha$ ) with a $131 \%$ increase over the control (Fig. 4.12B). It was further confirmed that phosphorylation of ACC/ACC, one of main downstream targets of AMPK $\alpha$, was consistently increased by chicoric acid treatment (Fig. 4.12C).

A.
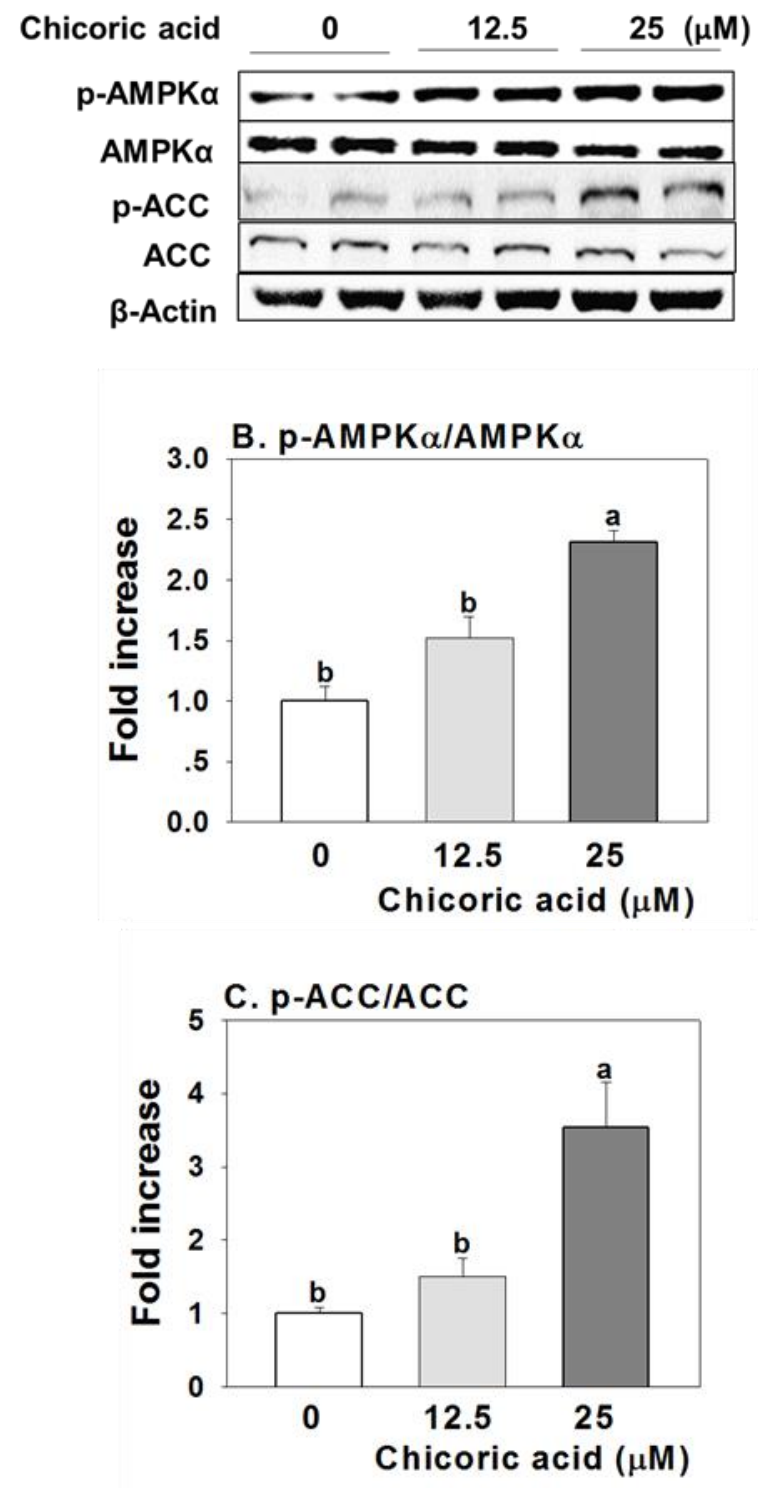

Figure 4.12 Activation of AMP-activated protein kinase $\alpha$ (AMPK $\alpha$ ) pathway by chicoric acid in $\mathrm{C} 2 \mathrm{C} 12$ myotubes. (A) Myotubes were treated with chicoric acid (12.5 $\mu \mathrm{M}$ or $25 \mu \mathrm{M}$ ) for $24 \mathrm{~h}$ in DMEM. Phosphorylation of AMPK $\alpha$ and ACC and the total were determined by immunoblotting. (B) p-AMPK $\alpha / A M P K \alpha$, phosphorylated AMPactivated protein kinase- $\alpha /$ AMP-activated protein kinase- $\alpha$. (C) p-ACC/ACC, 
phosphorylated acetyl-CoA carboxylase/acetyl-CoA carboxylase. Numbers are mean \pm S.E. $(\mathrm{n}=4)$. Means with different letters are significantly different at $P<0.05$.

Next, we further proved that activation of AMPK $\alpha$ is involved in the effects of chicoric acid on activation of Akt by using two approaches: an AMPK $\alpha$ inhibitor (Compound C) and AMPKa knockdown cells. Compound $\mathrm{C}$ was regarded as a selective AMPK $\alpha$ inhibitor with no influence on kinases that have similar structures with AMPK $\alpha$ (144). Therefore, we used Compound $\mathrm{C}$ first to determine whether Akt activation by chicoric acid is mediated by an AMPK $\alpha$-dependent pathway (144). Both Compound C and chicoric acid had significant effects on Akt phosphorylation, with a significant interaction between Compound $\mathrm{C}$ and chicoric acid $(P=0.049)$. The phosphorylation of AMPK $\alpha$ by chicoric acid was inhibited after treatment with Compound $\mathrm{C}(10 \mu \mathrm{M})$ as expected (Fig 4.13A-B). Chicoric acid consistently upregulated Akt phosphorylation without Compound $\mathrm{C}(P=0.003)$, while chicoric acid induced Akt activation was abolished by Compound C (Fig. 4.13A \& C). 

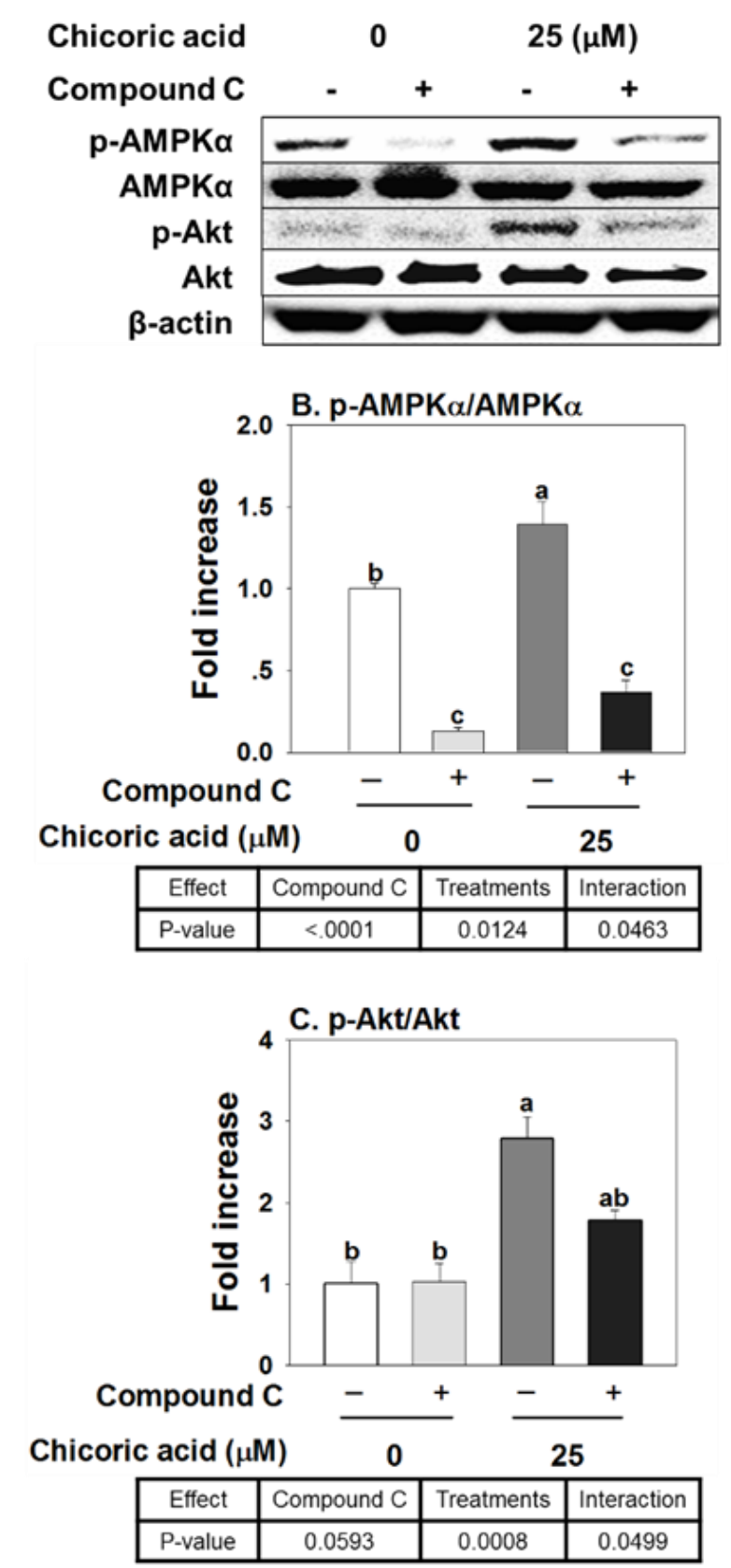

Figure 4.13 Increased glucose uptake and activation of Akt by chicoric acid were abolished by the inhibition of AMPK $\alpha$. (A) Cells were treated with $10 \mu \mathrm{M}$ compound $\mathrm{C}$ for $12 \mathrm{~h}$, then myotubes were treated with $25 \mu \mathrm{M}$ chicoric acid for $24 \mathrm{~h}$. (B) pAMPK $\alpha / A M P K \alpha$, phosphorylated AMP-activated protein kinase- $\alpha / A M P$-activated protein kinase- $\alpha$. (C) p-Akt/Akt, phosphorylated protein kinase B/protein kinase B. Numbers are mean \pm S.E. $(n=3-5)$. Means with different letters are significantly different at $P<0.05$. 
Next, we prepared AMPK $\alpha$ knockout cells to further confirm if chicoric acid had significant effects on Akt phosphorylation and glucose uptake. As shown in Fig. 4.14A and $\mathrm{B}$, knocking-out AMPK $\alpha$ abolished the effects of chicoric acid on the activation of Akt. Consistently, increased glucose uptake by chicoric acid was abolished in AMPK $\alpha$ knock-out cells (Fig. 4.14C). These results indicate that chicoric acid increases the phosphorylation of Akt and glucose uptake through an AMPKa-dependent pathway. 

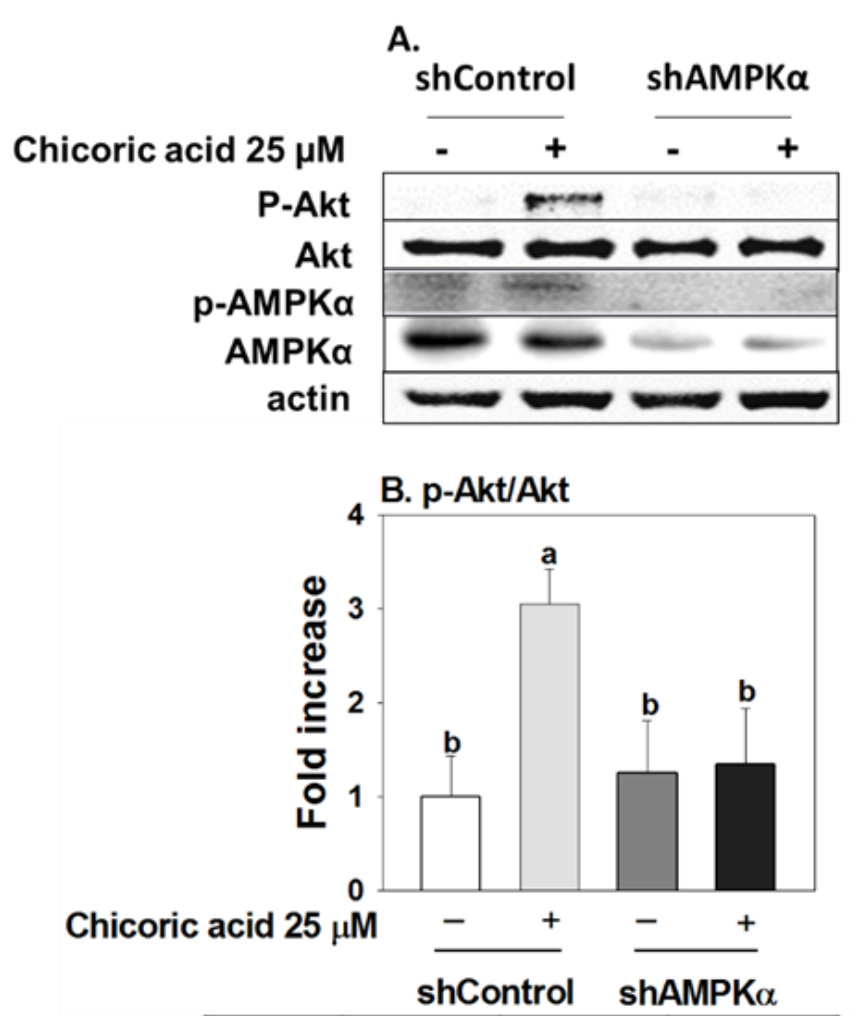

\begin{tabular}{|c|c|c|c|}
\hline Effect & shAMPKa & Treatments & Interaction \\
\hline P-value & 0.0532 & 0.0154 & 0.0208 \\
\hline
\end{tabular}

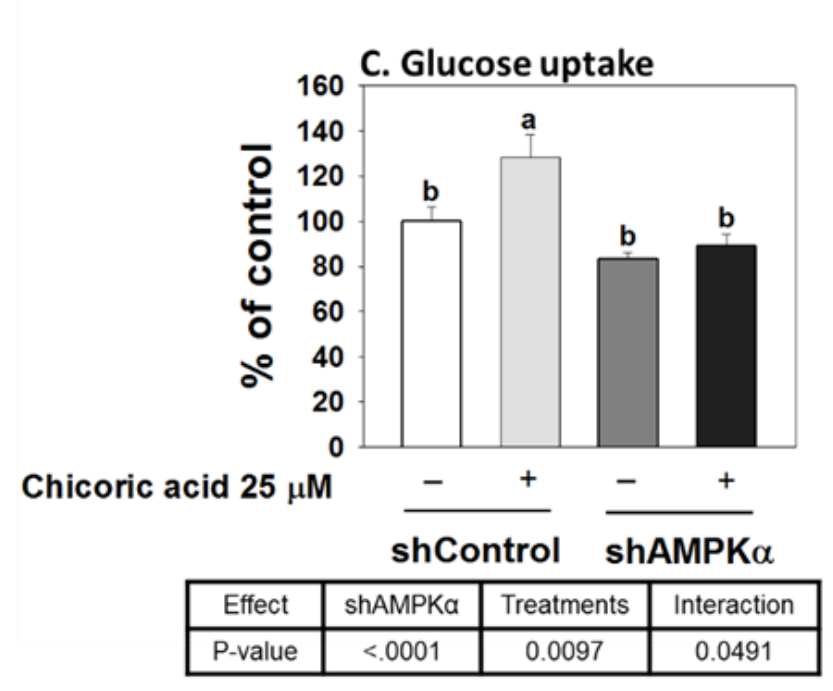

Figure 4.14 Increased glucose uptake and activation of Akt by chicoric acid were abolished by shAMPK $\alpha$. (A) Cells were infected with lentivirus vectors expressing shRNA targeting mouse AMPK $\alpha 1$. Phosphorylation of Akt and the total Akt were determined by immunoblotting. (B) p-Akt/Akt, phosphorylated protein kinase B/ protein kinase B. (C) Glucose levels were determined in C2C12 myotubes treated with 0 or 25 $\mu \mathrm{M}$ chicoric acid for $24 \mathrm{~h}$. Numbers are mean \pm S.E. $(n=3-5)$. Means with different letters are significantly different at $P<0.05$. 
In aerobic respiration, an increase in AMP level has been reported to directly induce AMPK $\alpha$ activation (151). Thus, we further determined if AMPK $\alpha$ activation by chicoric acid is attributed to the enhanced AMP/ATP ratio. Figure 4.15 showed that the AMP/ATP ratio was increased by $133 \%$ in the $25 \mu \mathrm{M}$ chicoric acid treatment group compared with the control $(P<0.001)$, which suggests that chicoric acid activates AMPK $\alpha$ via regulation of the AMP/ATP ratio.

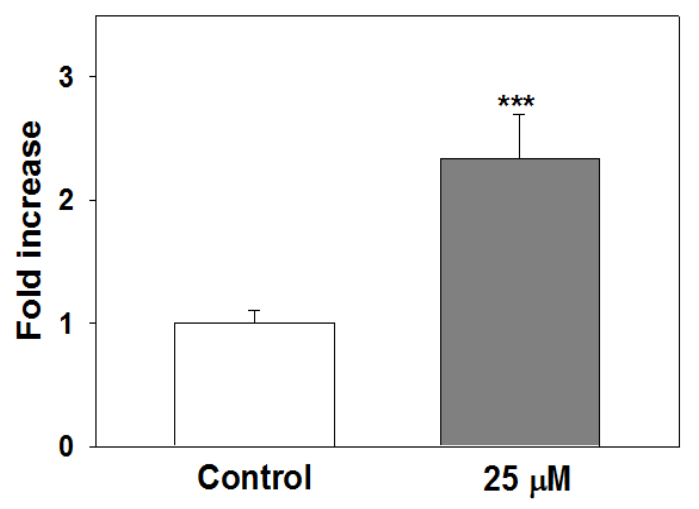

Figure 4.15 Chicoric acid enhanced AMP/ATP ratio in C2C12 myotubes. After $24 \mathrm{~h}$ of chicoric acid treatment, cells were lysed, and the supernatant lysate was taken to detect the levels of AMP and ATP using a commercial kit. The absorbance of each sample was determined by after incubation for $15 \mathrm{~min}$. Values are means \pm S.E. $(\mathrm{n}=3), * * * P<0.001$.

\subsubsection{Discussion}

Several phenolic secondary metabolites, such as chlorogenic acid and caffeic acid, have been used as antihyperglycemic compounds in pharmaceutical formulations (11, 137). Chicoric acid, a new phenolic ester, has been reported to have a beneficial effect on glucose transport in several studies $(51,52,59,73)$. Consistently, we demonstrated that chicoric acid increased glucose uptake and activated Akt, particularly at $25 \mu \mathrm{M}$, and these effects were dependent to AMPK $\alpha$, but not insulin, in $\mathrm{C} 2 \mathrm{C} 12$ myotubes. The 
hypoglycemic effects of chicoric acid were further confirmed in vivo using mice at $5 \mathrm{mg}$ chicoric acid/kg BW.

One in vivo study showed that chicoric acid ( $3 \mathrm{mg} / \mathrm{kg} \mathrm{BW}$ ) decreased the blood glucose level by $53 \%$ with a 120 min treatment in diabetic mice (59). Others reported that the extract from Cichorium intybus root, containing about 15 or $30 \mathrm{mg}$ chicoric acid/kg BW, significantly decreased blood glucose level after 4 days in male Wistar rats (152). This is consistent with the current study which found that chicoric acid can significantly reduce glucose levels in male mice. Zhu et al. (51) reported that chicoric acid ameliorated glucosamine-induced insulin resistance through enhanced glucose uptake in HepG2 cells. Our current results indicate that chicoric acid increased glucose uptake, independent of insulin, in $\mathrm{C} 2 \mathrm{C} 12$ myotubes $(51,149,153)$. However, the current results are inconsistent with Tousch et al. (11), who found chicoric acid to increase glucose uptake only with insulin stimulation in L6 skeletal muscle cells . The inconsistency between the current results and that of Tousch et al. might be due to the different statistical analysis methods used for the latter (i.e. one-way, vs. two-way ANOVA in the current study). Alternatively, the inconsistency may derive from: using different cell lines (mice vs. rat muscle cell lines); different determination methods for glucose uptake; or a different length of treatment time (2-NBDG with $24 \mathrm{~h}$ treatment in the current study vs. $\left[{ }^{3} \mathrm{H}\right]$ deoxyglucose with $1 \mathrm{~h}$ treatment in Tousch et al.).

$\mathrm{AMPK} \alpha$ is a master regulator of energy homeostasis by activating proteins in catabolism pathways while turning off biosynthetic pathways (154). In cellular respiration, an increase in AMP level relative to ATP activates AMPK $\alpha$, which further stimulates the acute upregulation of ATP production and the downregulation of non- 
essential energy expenditure (155). Previous evidence also suggests that AMPK $\alpha$ is an important regulator of glucose transport, especially in muscle tissue by phosphorylation of insulin receptors, independent of insulin (135). Moreover, chicoric acid was previously reported to activate $\mathrm{AMPK} \alpha$, which was suggested to be involved in the regulation of glucose metabolism in HepG2 cells (52). In our study, a different cell line (C2C12 myotubes) was used, and we further found that chicoric acid increased the cellular AMP/ATP ratio, which might contribute to AMPK $\alpha$ activation. Prior study has suggested that increased cellular AMP level may attributed to an increase in mitochondrial respiration without a corresponding increase in ATP synthesis by oxidative phosphorylation, a process described as uncoupling of oxidative phosphorylation (156). It was reported that some caffeic acid phenethyl esters (CAPEs), the derivatives of caffeic acid, activated AMPK $\alpha$ through uncoupling of oxidative phosphorylation-induced increase in AMP level $(156,157)$. Since chicoric acid is structurally similar to CAPEs, we speculate that the increased AMP/ATP ratio by chicoric acid may from a mechanism similar to that of CAPEs. However, this still needs to be determined in future study.

Along with its hypoglycemic effect, there are reports of chicoric acid having other functional properties, such as antioxidation, anti-inflammation, and anti-fatty liver activities $(63,84)$. Chicoric acid has been considered a potent antioxidant agent due to its effect on inhibiting the accumulation of reactive oxygen species and the generation of inflammatory cytokines, such as nitrogen oxide, interleukin 6 , and tumor necrosis factor$\alpha(51,64)$. Others have reported that chicoric acid promotes mitochondrial biogenesis by enhancing the citrate synthase activity and the deacetylation of peroxisome proliferatoractivated receptor- $\gamma$ coactivator, which is directly activated by $\operatorname{AMPK} \alpha(73,154)$. More 
information about such properties will help us better understand chicoric acid as a functional food bioactive.

In conclusion, chicoric acid promoted insulin-independent glucose uptake and Akt phosphorylation by post-translational regulation of $\mathrm{AMPK} \alpha$ in $\mathrm{C} 2 \mathrm{C} 12$ myotubes, and improved glucose tolerance in the mice model. The current study demonstrated that chicoric acid is a potential antidiabetic nutraceutical that may be used for the prevention or treatment of diabetes.

\section{Chicoric acid}

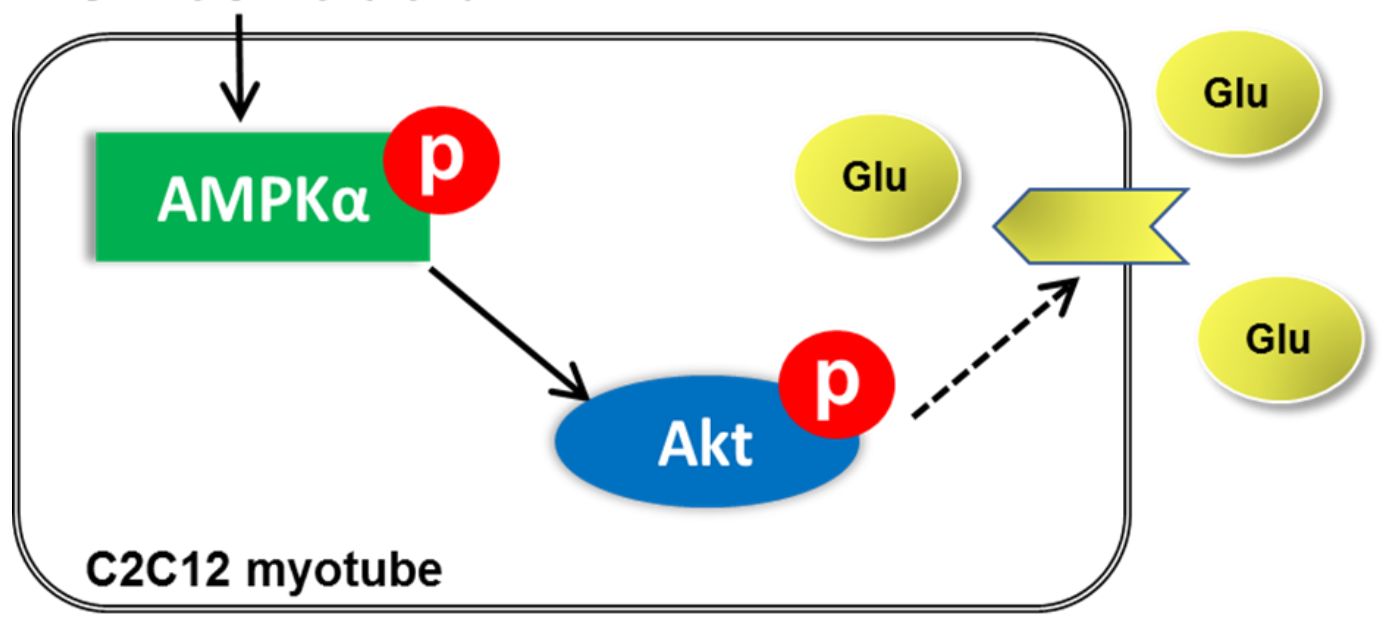

Fig. 4.16 The graphic contents. Chicoric acid promoted insulin-independent glucose uptake and Akt phosphorylation by post-translational regulation of AMPK $\alpha$ in $\mathrm{C} 2 \mathrm{C} 12$ myotubes. 


\section{BIBLIOGRAPHY}

1. Lee, J.; Scagel, C. F., Chicoric acid: chemistry, distribution, and production. Front. Chem. 2013, 31, 40.

2. Bauer, R., Chemistry, analysis and immunological investigations of Echinacea phytopharmaceuticals. In Immunomodulatory agents from plants, Springer: 1999; pp 4188.

3. $\quad$ Birt, D. F.; Widrlechner, M. P.; LaLone, C. A.; Wu, L.; Bae, J.; Solco, A. K. S.; Kraus, G. A.; Murphy, P. A.; Wurtele, E. S.; Leng, Q.; Hebert, S. C.; Maury, W. J.; Price, J. P., Echinacea in infection. Am. J. Clin. Nutr. 2008, 87, 488-492.

4. Zhang, Y.; Tang, T.; He, H.; Wu, H.; Hu, Z., Influence of several postharvest processing methods on polyphenol oxidase activity and cichoric acid content of Echinacea purpurea roots. Ind. Crop Prod. 2011, 34, 873-881.

5. Harman, D., The free radical theory of aging. Antioxid. Redox Signal. 2003, 5, $557-561$.

6. Niccoli, T.; Partridge, L., Ageing as a risk factor for disease. Curr. Biol. 2012, 22, 741-752.

7. Schlernitzauer, A.; Oiry, C.; Hamad, R.; Galas, S.; Cortade, F.; Chabi, B.; Casas, F.; Pessemesse, L.; Fouret, G.; Feillet-Coudray, C.; Cros, G.; Cabello, G.; Magous, R.; Wrutniak-Cabello, C., Chicoric acid is an antioxidant molecule that stimulates AMP kinase pathway in L6 myotubes and extends lifespan in Caenorhabditis elegans. PloS one 2013, 8, e78788.

8. $\quad$ Copeland, K. C.; Silverstein, J.; Moore, K. R.; Prazar, G. E.; Raymer, T.; Shiffman, R. N.; Springer, S. C.; Thaker, V. V.; Anderson, M.; Spann, S. J., Management of newly diagnosed type 2 diabetes mellitus (T2DM) in children and adolescents. Pediatrics 2013, 131, 364-382.

9. Donath, M. Y.; Shoelson, S. E., Type 2 diabetes as an inflammatory disease. Nat. Rev. Immunol. 2011, 11, 98-107.

10. Saltiel, A. R., New perspectives into the molecular pathogenesis and treatment of type 2 diabetes. Cell 2001, 104, 517-529.

11. Tousch, D.; Lajoix, A. D.; Hosy, E.; Azay-Milhau, J.; Ferrare, K.; Jahannault, C.; Cros, G.; Petit, P., Chicoric acid, a new compound able to enhance insulin release and glucose uptake. Biochemical and Biophysical Research Communications 2008, 377, 131135. 
12. Bais, H. P.; Ravishankar, G. A., Cichorium intybus L-cultivation, processing, utility, value addition and biotechnology, with an emphasis on current status and future prospects. J. Sci. Food Agric. 2001, 81, 467-484.

13. Street, R. A.; Sidana, J.; Prinsloo, G., Cichorium intybus: traditional uses, phytochemistry, pharmacology, and toxicology. Evid. Based Complement. Alternat. Med. 2013, 2013, 1-13.

14. Nandagopal, S.; Kumari, B. R., Phytochemical and antibacterial studies of Chicory (Cichorium intybus L.)-A multipurpose medicinal plant. Adv. Biol. Res. 2007, 1, $17-21$.

15. Barrett, B., Medicinal properties of Echinacea: A critical review. Phytomedicine 2003, 10, 66-86.

16. Robinson, W. E.; Reinecke, M. G.; AbdelMalek, S.; Jia, Q.; Chow, S. A., Inhibitors of HIV-1 replication that inhibit HPV integrase. Proc. Natl. Acad. Sci. U. S. A. 1996, 93, 6326-6331.

17. Beale, K. K.; Robinson, E. W., Combinations of reverse transcriptase, protease, and integrase inhibitors can be synergistic in vitro against drug-sensitive and RT inhibitor-resistant molecular clones of HIV-1. Antivir. Res. 2000, 46, 223-232.

18. Crosby, D. C.; Lei, X. Y.; Gibbs, C. G.; McDougall, B. R.; Robinson, W. E.; Reinecke, M. G., Design, synthesis, and biological evaluation of novel hybrid dicaffeoyltartaric/diketo acid and tetrazole-substituted 1-chicoric acid analogue inhibitors of human immunodeficiency virus type 1 Integrase. J. Med. Chem. 2010, 53, 8161-8175.

19. King, P. J.; Ma, G. X.; Miao, W. F.; Jia, Q.; McDougall, B. R.; Reinecke, M. G.; Cornell, C.; Kuan, J.; Kim, T. R.; Robinson, W. E., Structure-activity relationships: Analogues of the dicaffeoylquinic and dicaffeoyltartaric acids as potent inhibitors of human immunodeficiency virus type 1 integrase and replication. J. Med. Chem. 1999, 42, 497-509.

20. King, P. J.; Robinson, W. E., Resistance to the anti-human immunodeficiency virus type 1 compound L-chicoric acid results from a single mutation at amino acid 140 of integrase. J. Virol. 1998, 72, 8420-8424.

21. Reinke, R. A.; King, P. J.; Victoria, J. G.; McDougall, B. R.; Ma, G. X.; Mao, Y. Q.; Reinecke, M. G.; Robinson, W. E., Dicaffeoyltartaric acid analogues inhibit human immunodeficiency virus type 1 (HIV-1) integrase and HIV-1 replication at nontoxic concentrations. J. Med. Chem. 2002, 45, 3669-3683.

22. Reinke, R. A.; Lee, D. J.; McDougall, B. R.; King, P. J.; Victoria, J.; Mao, Y. Q.; Lei, X. Y.; Reinecke, M. G.; Robinson, W. E., L-chicoric acid inhibits human 
immunodeficiency virus type 1 integration in vivo and is a noncompetitive but reversible inhibitor of HIV-1 integrase in vitro. Virology 2004, 326, 203-219.

23. Robinson, W. E., L-chicoric acid, an inhibitor of human immunodeficiency virus type 1 (HIV-1) integrase, improves on the in vitro anti-HIV-l effect of Zidovudine plus a protease inhibitor (AG1350). Antivir. Res. 1998, 39, 101-111.

24. Chiu, T. K.; Davies, D. R., Structure and function of HIV-1 integrase. Curr. Top. Med. Chem. 2004, 4, 965-977.

25. Hu, J. P.; Chang, S.; Chen, W. Z.; Wang, C. X., Study on the drug resistance and the binding mode of HIV-1 integrase with LCA inhibitor. Sci. China Ser. B 2007, 50, 665-674.

26. King, P. J.; Lee, D. J.; Reinke, R. A.; Victoria, J. G.; Beale, K.; Robinson, W. E., Human immunodeficiency virus type-1 integrase containing a glycine to serine mutation at position 140 is attenuated for catalysis and resistant to integrase inhibitors. Virology 2003, 306, 147-161.

27. Healy, E. F.; Sanders, J.; King, P. J.; Robinson, W. E., A docking study of Lchicoric acid with HIV-1 integrase. J. Mol. Graph. Model. 2009, 27, 584-589.

28. Sahu, K. K.; Ravichandran, V.; Jain, P. K.; Sharma, S.; Mourya, V. K.; Agrawal, R. K., QSAR analysis of chicoric acid derivatives as HIV-1 integrase inhibitors. Acta. Chim. Slov. 2008, 55, 138-145.

29. McDougall, B.; King, P. J.; Wu, B. W.; Hostomsky, Z.; Reinecke, M. G.; Robinson, W. E., Jr., Dicaffeoylquinic and dicaffeoyltartaric acids are selective inhibitors of human immunodeficiency virus type 1 integrase. Antimicrob Agents Chemother 1998, $42,140-146$.

30. Charvat, T. T.; Lee, D. J.; Robinson, W. E.; Chamberlin, A. R., Design, synthesis, and biological evaluation of chicoric acid analogs as inhibitors of HIV-1 integrase. Bioorganic Med. Chem. 2006, 14, 4552-4567.

31. Meadows, D. C.; Mathews, T. B.; North, T. W.; Hadd, M. J.; Kuo, C. L.; Neamati, N.; Gervay-Hague, J., Synthesis and biological evaluation of geminal disulfones as HIV1 integrase inhibitors. J. Med. Chem. 2005, 48, 4526-4534.

32. Meadows, D. C.; Sanchez, T.; Neamati, N.; North, T. W.; Gervay-Hague, J., Ring substituent effects on biological activity of vinyl sulfones as inhibitors of HIV-1.

Bioorganic Med. Chem. 2007, 15, 1127-1137.

33. Liu, Q.; Chen, Y. W.; Shen, C.; Xiao, Y. T.; Wang, Y. T.; Liu, Z. G.; Liu, X. B., Chicoric acid supplementation prevents systemic inflammation- induced memory 
impairment and amyloidogenesis via inhibition of NF-kappa B. Faseb J. 2017, 31, 14941507.

34. Liu, Q.; Hu, Y. Y.; Cao, Y. F.; Song, G.; Liu, Z. G.; Liu, X. B., Chicoric acid ameliorates lipopolysaccharide-lnduced oxidative stress via promoting the Keapl/Nrf2 transcriptional signaling pathway in BV-2 Microglial cells and mouse brain. J. Agric. Food Chem. 2017, 65, 338-347.

35. Matthias, A.; Banbury, L.; Stevenson, L. M.; Bone, K. M.; Leach, D. N.; Lehmann, R. P., Alkylamides from Echinacea modulate induced immune responses in macrophages. Immunol. Invest. 2007, 36, 117-130.

36. Stevenson, L. M.; Matthias, A.; Banbury, L.; Penman, K. G.; Bone, K. M.; Leach, D. L.; Lehmann, R. P., Modulation of macrophage immune responses by Echinacea. Molecules 2005, 10, 1279-1285.

37. Brasier, A. R., The NF-кB regulatory network. Cardiovasc. Toxicol. 2006, 6, 111130.

38. Gilmore, T. D., Introduction to NF-kB: players, pathways, perspectives. Oncogene 2006, 25, 6680.

39. Zheng, X. X.; Maslinski, W.; Ferrari-Lacraz, S.; Strom, T. B., Cytokines in the Treatment and Prevention of Autoimmune Responses. 2013.

40. Jiang, L.; Li, W. Z.; Wang, Y. C.; Zhang, X. S.; Yu, D. Q.; Yin, Y. Y.; Xie, Z. W.; Yuan, Y., Effects of cichoric acid extract from Echinacea purpurea on collagen-induced arthritis in rats. Am. J. Chinese Med. 2014, 42, 679-692.

41. Kour, K.; Bani, S.; Sangwan, P. L.; Singh, A., Upregulation of Th1 polarization by Taraxacum officinale in normal and immune suppressed mice. Curr. Sci. 2016, 111, 671-685.

42. Tsai, K. L.; Kao, C. L.; Hung, C. H.; Cheng, Y. H.; Lin, H. C.; Chu, P. M., Chicoric acid is a potent anti-atherosclerotic ingredient by anti-oxidant action and antiinflammation capacity. Oncotarget 2017, 8, 29600-29612.

43. Xue, Y. S.; Zhang, S. M.; Du, M.; Zhu, M. J., Dandelion extract suppresses reactive oxidative species and inflammasome in intestinal epithelial cells. J. Funct. Foods 2017, 29, 10-18.

44. Matthias, A.; Banbury, L.; Bone, K. M.; Leach, D. N.; Lehmann, R. P., Echinacea alkylamides modulate induced immune responses in T-cells. Fitoterapia 2008, 79, 53-58. 
45. Goel, V.; Chang, C.; Slama, J. V.; Barton, R.; Bauer, R.; Gahler, R.; Basu, T. K., Echinacea stimulates macrophage function in the lung and spleen of normal rats. J. Nutr. Biochem. 2002, 13, 487-492.

46. Johnson, R. W., Inhibition of growth by pro-inflammatory cytokines: An integrated view. J. Aanim. Sci. 1997, 75, 1244-1255.

47. Park, C. M.; Jin, K. S.; Lee, Y. W.; Song, Y. S., Luteolin and chicoric acid synergistically inhibited inflammatory responses via inactivation of PI3K-Akt pathway and impairment of NF-kappa B translocation in LPS stimulated RAW 264.7 cells. Eur. J. Pharmacol. 2011, 660, 454-459.

48. Park, C. M.; Park, J. Y.; Noh, K. H.; Shin, J. H.; Song, Y. S., Taraxacum officinale Weber extracts inhibit LPS-induced oxidative stress and nitric oxide production via the NF-kappa B modulation in RAW 264.7 cells. J. Ethnopharmacol. 2011, 133, 834842.

49. Gertsch, J.; Schoop, R.; Kuenzle, U.; Suter, A., Echinacea alkylamides modulate TNF- $\alpha$ gene expression via cannabinoid receptor CB2 and multiple signal transduction pathways. FEBS Letters 2004, 577, 563-569.

50. Azay-Milhau, J.; Ferrare, K.; Leroy, J.; Aubaterre, J.; Tournier, M.; Lajoix, A. D.; Tousch, D., Antihyperglycemic effect of a natural chicoric acid extract of chicory (Cichorium intybus L.): A comparative in vitro study with the effects of caffeic and ferulic acids. Journal of Ethnopharmacology 2013, 150, 755-760.

51. Zhu, D.; Wang, Y. T.; Du, Q. W.; Liu, Z. G.; Liu, X. B., Cichoric acid reverses insulin resistance and suppresses inflammatory responses in the glucosamine-induced HepG2 cells. J. Agric. Food Chem. 2015, 63, 10903-10913.

52. Zhu, D.; Zhang, N.; Zhou, X. L.; Zhang, M. Y.; Liu, Z. G.; Liu, X. B., Cichoric acid regulates the hepatic glucose homeostasis via AMPK pathway and activates the antioxidant response in high glucose-induced hepatocyte injury. $R S C A d v$. 2017, 7, 13631375.

53. Dalar, A.; Konczak, I., Cichorium intybus from Eastern Anatolia: Phenolic composition, antioxidant and enzyme inhibitory activities. Ind. Crops Prod. 2014, 60, 7985 .

54. McCue, P.; Kwon, Y.-I.; Shetty, K., Anti-diabetic and anti-hypertensive potential of sprouted and solid-state bioprocessed soybean. Asia Pac. J. Clin. Nutr. 2005, 14, 145.

55. Zhang, A. J.; Rimando, A. M.; Mizuno, C. S.; Mathews, S. T., $\alpha$-Glucosidase inhibitory effect of resveratrol and piceatannol. J. Nutr. Biochem. 2017, 47, 86-93. 
56. Zhang, Z. Y., Protein tyrosine phosphatases: Prospects for therapeutics. Curr. Opin. Chem. Biol. 2001, 5, 416-423.

57. Baskaran, S. K.; Goswami, N.; Selvaraj, S.; Muthusamy, V. S.; Lakshmi, B. S., Molecular dynamics approach to probe the allosteric inhibition of PTP1B by chlorogenic and cichoric acid. J. Chem. Inf. Model 2012, 52, 2004-2012.

58. Lipchock, J. M.; Hendrickson, H. P.; Douglas, B. B.; Bird, K. E.; Ginther, P. S.; Rivalta, I.; Ten, N. S.; Batista, V. S.; Loria, J. P., Characterization of Protein Tyrosine Phosphatase 1B Inhibition by Chlorogenic Acid and Cichoric Acid. Biochemistry 2017, 56, 96-106.

59. Casanova, L. M.; da Silva, D.; Sola-Penna, M.; Camargo, L. M. D.; Celestrini, D. D.; Tinoco, L. W.; Costa, S. S., Identification of chicoric acid as a hypoglycemic agent from Ocimum gratissimum leaf extract in a biomonitoring in vivo study. Fitoterapia 2014, 93, 132-141.

60. Casanova, L. M.; Espindola-Netto, J. M.; Tinoco, L. W.; Sola-Penna, M.; Costa, S. $\mathrm{S}$., The use of NMR metabolite profiling and in vivo hypoglycemic assay for comparison of unfractionated aqueous leaf extracts of two Ocimum species. Chem. Biodivers. 2016, $13,686-694$.

61. Zhu, D.; Zhang, X.; Niu, Y.; Diao, Z.; Ren, B.; Li, X.; Liu, Z.; Liu, X., Cichoric acid improved hyperglycaemia and restored muscle injury via activating antioxidant response in MLD-STZ-induced diabetic mice. Food Chem. Toxicol. 2017, 107, 138-149.

62. Lyon, C. J.; Law, R. E.; Hsueh, W. A., Minireview: Adiposity, inflammation, and atherogenesis. Endocrinology 2003, 144, 2195-2200.

63. Kim, M.; Yoo, G.; Randy, A.; Kim, H. S.; Nho, C. W., Chicoric acid attenuate a nonalcoholic steatohepatitis by inhibiting key regulators of lipid metabolism, fibrosis, oxidation, and inflammation in mice with methionine and choline deficiency. Mol. Nutr. Food Res. 2017, 61, 1-14.

64. Xiao, H. F.; Xie, G.; Wang, J. W.; Hou, X. F.; Wang, X.; Wu, W. Q.; Liu, X. B., Chicoric acid prevents obesity by attenuating hepatic steatosis, inflammation and oxidative stress in high-fat diet-fed mice. Food Res. Int. 2013, 54, 345-353.

65. Landmann, M.; Kanuri, G.; Spruss, A.; Stahl, C.; Bergheim, I., Oral intake of chicoric acid reduces acute alcohol-induced hepatic steatosis in mice. Nutrition 2014, 30, 882-889.

66. Kour, K.; Bani, S., Chicoric acid regulates behavioral and biochemical alterations induced by chronic stress in experimental Swiss albino mice. Pharmacol. Biochem. Behav. 2011, 99, 342-348. 
67. Ahn, H. R.; Lee, H. J.; Kim, K. A.; Kim, C. Y.; Nho, C. W.; Jang, H.; Pan, C. H.; Lee, C. Y.; Jung, S. H., Hydroxycinnamic acids in Crepidiastrum denticulatum protect oxidative stress-induced retinal damage. J. Agric. Food Chem. 2014, 62, 1310-1323.

68. Ogomori, K.; Kitamoto, T.; Tateishi, J.; Sato, Y.; Suetsugu, M.; Abe, M., Betaprotein amyloid is widely distributed in the central nervous system of patients with Alzheimer's disease. Am. J. Pathol. 1989, 134, 243.

69. Bekinschtein, P.; Cammarota, M.; Katche, C.; Slipczuk, L.; Rossato, J. I.; Goldin, A.; Izquierdo, I.; Medina, J. H., BDNF is essential to promote persistence of long-term memory storage. PNAS 2008, 105, 2711-2716.

70. Finkel, T.; Holbrook, N. J., Oxidants, oxidative stress and the biology of ageing. Nature 2000, 408, 239.

71. Apel, K.; Hirt, H., Reactive oxygen species: metabolism, oxidative stress, and signal transduction. Annu. Rev. Plant Biol. 2004, 55, 373-399.

72. Kimura, T.; Yamamoto, S.; Ogawa, I.; Miura, H.; Hasegawa, M., Antioxidant ability of chicoric acid and its analogous compounds. Nippon Kagaku Kaishi 1999, 739750 .

73. Schlernitzauer, A.; Oiry, C.; Hamad, R.; Galas, S.; Cortade, F.; Chabi, B.; Casas, F.; Pessemesse, L.; Fouret, G.; Feillet-Coudray, C.; Cros, G.; Cabello, G.; Magous, R.; Wrutniak-Cabello, C., Chicoric Acid Is an Antioxidant Molecule That Stimulates AMP Kinase Pathway in L6 Myotubes and Extends Lifespan in Caenorhabditis elegans. PloS one 2013, 8 .

74. Thygesen, L.; Thulin, J.; Mortensen, A.; Skibsted, L. H.; Molgaard, P., Antioxidant activity of cichoric acid and alkamides from Echinacea purpurea, alone and in combination. Food Chem. 2007, 101, 74-81.

75. Wang, Y. T.; Diao, Z. J.; Li, J.; Ren, B.; Zhu, D.; Liu, Q.; Liu, Z. G.; Liu, X. B., Chicoric acid supplementation ameliorates cognitive impairment induced by oxidative stress via promotion of antioxidant defense system. $R S C A d v$. 2017, 7, 36149-36162.

76. Liu, Q.; Wang, Y. T.; Xiao, C. X.; Wu, W. Q.; Liu, X. B., Metabolism of chicoric acid by rat liver microsomes and bioactivity comparisons of chicoric acid and its metabolites. Food Funct. 2015, 6, 1928-1935.

77. Montagna, W.; Carlisle, K., Structural changes in aging human skin. J. Investig. Dermatol. 1979, 73, 47-53.

78. Ma, Q., Role of nrf2 in oxidative stress and toxicity. Annu. Rev. Pharmacol. Toxicol. 2013, 53, 401-426. 
79. Valle, I.; Álvarez-Barrientos, A.; Arza, E.; Lamas, S.; Monsalve, M., PGC-1 $\alpha$ regulates the mitochondrial antioxidant defense system in vascular endothelial cells. Cardiovasc. Res. 2005, 66, 562-573.

80. Tsai, Y. L.; Chiu, C. C.; Yi-Fu Chen, J.; Chan, K. C.; Lin, S. D., Cytotoxic effects of Echinacea purpurea flower extracts and cichoric acid on human colon cancer cells through induction of apoptosis. J. Ethnopharmacol. 2012, 143, 914-919.

81. Segueni, N.; Magid, A. A.; Decarme, M.; Rhouati, S.; Lahouel, M.; Antonicelli, F.; Lavaud, C.; Hornebeck, W., Inhibition of stromelysin-1 by caffeic acid derivatives from a propolis sample from Algeria. Planta. Medica. 2011, 77, 999-1004.

82. Lin, Z. W.; Neamati, N.; Zhao, H.; Kiryu, Y.; Turpin, J. A.; Aberham, C.; Strebel, K.; Kohn, K.; Witvrouw, M.; Pannecouque, C.; Debyser, Z.; De Clercq, E.; Rice, W. G.; Pommier, Y.; Burke, T. R., Chicoric acid analogues as HIV-1 integrase inhibitors. J. Med. Chem. 1999, 42, 1401-1414.

83. Pluymers, W.; Neamati, N.; Pannecouque, C.; Fikkert, V.; Marchand, C.; Burke, T. R.; Pommier, Y.; Schols, D.; De Clercq, E.; Debyser, Z.; Witvrouw, M., Viral entry as the primary target for the anti-HIV activity of chicoric acid and its tetra-acetyl esters. Mol. Pharmacol. 2000, 58, 641-648.

84. Dalby-Brown, L.; Barsett, H.; Landbo, A. K. R.; Meyer, A. S.; Molgaard, P., Synergistic antioxidative effects of alkamides, caffeic acid derivatives, and polysaccharide fractions from Echinacea purpurea on in vitro oxidation of human lowdensity lipoproteins. J. Agric. Food Chem. 2005, 53, 9413-9423.

85. Chen, L. X.; Huang, G.; Gao, M.; Shen, X. D.; Gong, W.; Xu, Z. Z.; Zeng, Y. J.; He, F. T., Chicoric acid suppresses BAFF expression in B lymphocytes by inhibiting NFkappa B activity. Int. Immunopharmacol. 2017, 44, 211-215.

86. Zhang, H. L.; Dai, L. H.; Wu, Y. H.; Yu, X. P.; Zhang, Y. Y.; Guan, R. F.; Liu, T.; Zhao, J., Evaluation of hepatocyteprotective and anti-hepatitis B virus properties of cichoric acid from Cichorium intybus leaves in cell culture. Biol. Pharm. Bull. 2014, 37, 1214-1220.

87. Huntimer, E. D.; Halaweish, F. T.; Chase, C. C. L., Proliferative activity of Echinacea angustifolia root extracts on cancer cells: Interference with doxorubicin cytotoxicity. Chem. Biodivers. 2006, 3, 695-703.

88. Kour, K.; Bani, S., Augmentation of immune response by chicoric acid through the modulation of CD28/CTLA-4 and Th1 pathway in chronically stressed mice. Neuropharmacology 2011, 60, 852-860. 
89. Lee, N. Y.; Chung, K. S.; Jin, J. S.; Bang, K. S.; Eom, Y. J.; Hong, C. H.; Nugroho, A.; Park, H. J.; An, H. J., Effect of chicoric acid on mast cell-mediated allergic inflammation in vitro and in vivo. J. Nat. Prod. 2015, 78, 2956-2962.

90. Perkins, N. D., Integrating cell-signalling pathways with NF- $\kappa \mathrm{B}$ and IKK function. Nat. Rev. Mol. Cell Bio. 2007, 8, 49.

91. Wang, Y. T.; Xie, G.; Liu, Q.; Duan, X.; Liu, Z. G.; Liu, X. B., Pharmacokinetics, tissue distribution, and plasma protein binding study of chicoric acid by HPLC-MS/MS. $J$. Chromatogr. B 2016, 1031, 139-145.

92. Lee, K. S.; Lee, B. S.; Semnani, S.; Avanesian, A.; Um, C. Y.; Jeon, H. J.; Seong, K. M.; Yu, K.; Min, K. J.; Jafari, M., Curcumin extends life span, improves health span, and modulates the expression of age-associated aging genes in Drosophila melanogaster. Rejuvenation Res. 2010, 13, 561-570.

93. Rathor, L.; Akhoon, B. A.; Pandey, S.; Srivastava, S.; Pandey, R., Folic acid supplementation at lower doses increases oxidative stress resistance and longevity in Caenorhabditis elegans. Age 2015, 37, 113.

94. Shen, P.; Yue, Y.; Sun, Q.; Kasireddy, N.; Kim, K. H.; Park, Y., Piceatannol extends the lifespan of Caenorhabditis elegans via DAF-16. BioFactors 2017, 43, 379387.

95. Zheng, S. Q.; Huang, X. B.; Xing, T. K.; Ding, A. J.; Wu, G. S.; Luo, H. R., Chlorogenic acid extends the lifespan of Caenorhabditis elegans via insulin/IGF-1 signaling pathway. J. Gerontol. A. Biol. Sci. Med. Sci. 2017, 72, 464-472.

96. Hart, A. C.; Onken, B.; Driscoll, M., Metformin induces a dietary restriction-like state and the oxidative stress response to extend C. elegans healthspan via AMPK, LKB1, and SKN-1. PloS one 2010, 5, e8758.

97. Hwang, A. B.; Ryu, E.-A.; Artan, M.; Chang, H.-W.; Kabir, M. H.; Nam, H.-J.; Lee, D.; Yang, J.-S.; Kim, S.; Mair, W. B., Feedback regulation via AMPK and HIF-1 mediates ROS-dependent longevity in Caenorhabditis elegans. PNAS 2014, 111, 44584467.

98. Salminen, A.; Kaarniranta, K., AMP-activated protein kinase (AMPK) controls the aging process via an integrated signaling network. Ageing Res. Rev. 2012, 11, 230241.

99. Tullet, J. M.; Hertweck, M.; An, J. H.; Baker, J.; Hwang, J. Y.; Liu, S.; Oliveira, R. P.; Baumeister, R.; Blackwell, T. K., Direct inhibition of the longevity-promoting factor SKN-1 by insulin-like signaling in C. elegans. Cell 2008, 132, 1025-1038. 
100. Stiernagle, T., Maintenance of C. elegans. WormBook: the online review of $C$. elegans biology 2006, 1-11.

101. Sun, Q.; Yue, Y.; Shen, P.; Yang, J. J.; Park, Y., Cranberry product decreases fat accumulation in Caenorhabditis elegans. J. Med. Food 2016, 19, 427-433.

102. Shen, P.; Yue, Y.; Zheng, J.; Park, Y., Caenorhabditis elegans: A convenient in vivo model for assessing the impact of food bioactive compounds on obesity, aging, and Alzheimer's disease. Annu. Rev. Food Sci. Technol. 2018, 9, 1-22.

103. Yue, Y.; Shen, P.; Xu, Y.; Park, Y., p-Coumaric acid improves oxidative and osmosis stress responses in Caenorhabditis elegans. J. Sci. Food Agric. 2018, 99, 11901197.

104. Colmenares, D.; Sun, Q.; Shen, P.; Yue, Y.; McClements, D. J.; Park, Y., Delivery of dietary triglycerides to Caenorhabditis elegans using lipid nanoparticles: Nanoemulsion-based delivery systems. Food Chem. 2016, 202, 451-457.

105. Chen, Y.; Onken, B.; Chen, H.; Xiao, S.; Liu, X.; Driscoll, M.; Cao, Y.; Huang, Q., Mechanism of longevity extension of Caenorhabditis elegans induced by pentagalloyl glucose isolated from eucalyptus leaves. J Agric. Food Chem. 2014, 62, 3422-3431.

106. Jeong, D. E.; Lee, Y.; Lee, S. J. V., Western blot analysis of C. elegans proteins. In Hypoxia, Springer: 2018; pp 213-225.

107. Sun, Q.; Zhang, Z.; Zhang, R.; Gao, R.; McClements, D. J., Development of functional or medical foods for oral administration of insulin for diabetes treatment: Gastroprotective edible microgels. J Agric. Food Chem. 2018, 66, 4820-4826.

108. Apfeld, J.; O'Connor, G.; McDonagh, T.; DiStefano, P. S.; Curtis, R., The AMPactivated protein kinase AAK-2 links energy levels and insulin-like signals to lifespan in C. elegans. Gene Dev. 2004, 18, 3004-3009.

109. Mair, W.; Morantte, I.; Rodrigues, A. P.; Manning, G.; Montminy, M.; Shaw, R. J.; Dillin, A., Lifespan extension induced by AMPK and calcineurin is mediated by CRTC-1 and CREB. Nature 2011, 470, 404.

110. Sagi, D.; Kim, S. K., An engineering approach to extending lifespan in C. elegans. PLoS Genet. 2012, 8, e1002780.

111. Kenyon, C.; Chang, J.; Gensch, E.; Rudner, A.; Tabtiang, R., A C. elegans mutant that lives twice as long as wild type. Nature 1993, 366, 461. 
112. Kimura, K. D.; Tissenbaum, H. A.; Liu, Y.; Ruvkun, G., daf-2, an insulin receptor-like gene that regulates longevity and diapause in Caenorhabditis elegans. Science 1997, 277, 942-946.

113. Dorman, J. B.; Albinder, B.; Shroyer, T.; Kenyon, C., The Age-1 and Daf-2 genes function in a common pathway to control the lifespan of Caenorhabditis elegans.

Genetics 1995, 141, 1399-1406.

114. Vowels, J. J.; Thomas, J. H., Genetic analysis of chemosensory control of dauer formation in Caenorhabditis elegans. Genetics 1992, 130, 105-123.

115. An, J. H.; Blackwell, T. K., SKN-1 links C. elegans mesendodermal specification to a conserved oxidative stress response. Gene Dev. 2003, 17, 1882-1893.

116. Bowerman, B.; Eaton, B. A.; Priess, J. R., skn-1, a maternally expressed gene required to specify the fate of ventral blastomeres in the early $C$. elegans embryo. Cell 1992, 68, 1061-1075.

117. Lakowski, B.; Hekimi, S., The genetics of caloric restriction in Caenorhabditis elegans. PNAS 1998, 95, 13091-13096.

118. Chang, H. W.; Pisano, S.; Chaturbedi, A.; Chen, J.; Gordon, S.; Baruah, A.; Lee, S. S., Transcription factors CEP - 1/p53 and CEH - 23 collaborate with AAK - 2/AMPK to modulate longevity in Caenorhabditis elegans. Aging Cell 2017, 16, 814-824.

119. Lee, H.; Cho, J. S.; Lambacher, N.; Lee, J.; Lee, S. J.; Lee, T. H.; Gartner, A.; Koo, H. S., The Caenorhabditis elegans AMP-activated protein kinase AAK-2 is phosphorylated by LKB1 and is required for resistance to oxidative stress and for normal motility and foraging behavior. J. Biol. Chem. 2008, 283, 14988-14993.

120. Moreno-Arriola, E.; Hafidi, M. E.; Ortega-Cuéllar, D.; Carvajal, K., AMPactivated protein kinase regulates oxidative metabolism in Caenorhabditis elegans through the NHR-49 and MDT-15 transcriptional regulators. PloS one 2016, 11, e0148089.

121. Li, H.; Liu, X.; Wang, D.; Su, L.; Zhao, T.; Li, Z.; Lin, C.; Zhang, Y.; Huang, B.; Lu, J., O-GlcNAcylation of SKN-1 modulates the lifespan and oxidative stress resistance in Caenorhabditis elegans. Sci. Rep. 2017, 7, 43601.

122. Blackwell, T. K.; Steinbaugh, M. J.; Hourihan, J. M.; Ewald, C. Y.; Isik, M., SKN-1/Nrf, stress responses, and aging in Caenorhabditis elegans. Free Radic. Biol. Med. 2015, 88, 290-301.

123. Chondrogianni, N.; Georgila, K.; Kourtis, N.; Tavernarakis, N.; Gonos, E. S., $20 \mathrm{~S}$ proteasome activation promotes life span extension and resistance to proteotoxicity in 
Caenorhabditis elegans. FASEB journal : official publication of the Federation of American Societies for Experimental Biology 2015, 29, 611-622.

124. Lüersen, K.; Stegehake, D.; Daniel, J.; Drescher, M.; Ajonina, I.; Ajonina, C.; Hertel, P.; Woltersdorf, C.; Liebau, E., The glutathione reductase GSR-1 determines stress tolerance and longevity in Caenorhabditis elegans. PloS one 2013, 8, e60731.

125. Harman, D., Aging: A theory based on free radical and radiation chemistry. Sci. Aging Knowledge Environ. 2002, 2002, 14.

126. Maeda, A.; Kai, K.; Ishii, M.; Ishii, T.; Akagawa, M., Safranal, a novel protein tyrosine phosphatase $1 \mathrm{~B}$ inhibitor, activates insulin signaling in C2C12 myotubes and improves glucose tolerance in diabetic KK - Ay mice. Mol. Nutr. Food Res. 2014, 58, 1177-1189.

127. Sykiotis, G. P.; Bohmann, D., Stress-activated cap'n'collar transcription factors in aging and human disease. Sci. Signal. 2010, 3, 1-45.

128. An, J. H.; Vranas, K.; Lucke, M.; Inoue, H.; Hisamoto, N.; Matsumoto, K.; Blackwell, T. K., Regulation of the Caenorhabditis elegans oxidative stress defense protein SKN-1 by glycogen synthase kinase-3. PNAS 2005, 102, 16275-16280.

129. Palikaras, K.; Lionaki, E.; Tavernarakis, N., Coordination of mitophagy and mitochondrial biogenesis during ageing in C. elegans. Nature 2015, 521, 525.

130. Bjornholm, M.; Zierath, J. R., Insulin signal transduction in human skeletal muscle: identifying the defects in type II diabetes. Biochem. Soci. Transact. 2005, 33, 354-357.

131. Kahn, S. E.; Hull, R. L.; Utzschneider, K. M., Mechanisms linking obesity to insulin resistance and type 2 diabetes. Nature 2006, 444, 840-846.

132. Wijesekara, N.; Thong, F. S. L.; Anonescu, C. N.; Klip, A., Diverse signals regulate glucose uptake into skeletal muscle. Can. J. Diabetes 2006, 30, 80-88.

133. Carling, D., The AMP-activated protein kinase cascade--a unifying system for energy control. Trends Biochem. Sci. 2004, 29, 18-24.

134. Fujii, N.; Jessen, N.; Goodyear, L. J., AMP-activated protein kinase and the regulation of glucose transport. Am. J. Physiol. Endocrinol. Metab. 2006, 291, 867-877.

135. Chopra, I.; Li, H. F.; Wang, H.; Webster, K. A., Phosphorylation of the insulin receptor by AMP-activated protein kinase (AMPK) promotes ligand-independent activation of the insulin signalling pathway in rodent muscle. Diabetologia 2012, 55, 783-794. 
136. Tao, R.; Gong, J.; Luo, X.; Zang, M.; Guo, W.; Wen, R.; Luo, Z., AMPK exerts dual regulatory effects on the PI3K pathway. J. Mol. Signal. 2010, 5, 1-9.

137. Meng, S.; Cao, J.; Feng, Q.; Peng, J.; Hu, Y., Roles of chlorogenic acid on regulating glucose and lipids metabolism: A review. Evid-Based Compl. Alt. 2013, 2013, $1-11$.

138. Innocenti, M.; Gallori, S.; Giaccherini, C.; Ieri, F.; Vincieri, F. F.; Mulinacci, N., Evaluation of the phenolic content in the aerial parts of different varieties of Cichorium intybus L. J. Agric. Food Chem. 2005, 53, 6497-6502.

139. Lee, J.; Scagel, C. F., Chicoric acid found in basil (Ocimum basilicum L.) leaves. Food Chem. 2009, 115, 650-656.

140. DeFronzo, R. A.; Gunnarsson, R.; Bjorkman, O.; Olsson, M.; Wahren, J., Effects of insulin on peripheral and splanchnic glucose metabolism in noninsulin-dependent (type II) diabetes mellitus. J. Clinic. Investig. 1985, 76, 149-155.

141. Deshmukh, A. S.; Murgia, M.; Nagaraj, N.; Treebak, J. T.; Cox, J.; Mann, M., Deep proteomics of mouse skeletal muscle enables quantitation of protein isoforms, metabolic pathways, and transcription factors. Mol. Cell. Proteom. 2015, 14, 841-853.

142. Sharples, A. P.; Al-Shanti, N.; Stewart, C. E., C2 and C2C12 murine skeletal myoblast models of atrophic and hypertrophic potential: relevance to disease and aging. $J$. Cell. Physiol. 2010, 225, 240-250.

143. Kim, J.; Park, Y.; Yoon, K. S.; Clark, J. M.; Park, Y., Permethrin alters adipogenesis in 3T3-L1 adipocytes and causes insulin resistance in C2C12 myotubes. $J$. Biochem. Mol. Toxicol. 2014, 28, 418-424.

144. Zhou, G.; Myers, R.; Li, Y.; Chen, Y.; Shen, X.; Fenyk-Melody, J.; Wu, M.; Ventre, J.; Doebber, T.; Fujii, N.; Musi, N.; Hirshman, M. F.; Goodyear, L. J.; Moller, D. E., Role of AMP-activated protein kinase in mechanism of metformin action. J. Clinic. Invest. 2001, 108, 1167-1174.

145. Xiao, H.; Wang, J.; Yuan, L.; Xiao, C.; Wang, Y.; Liu, X., Chicoric acid induces apoptosis in 3T3-L1 preadipocytes through ROS-mediated PI3K/Akt and MAPK signaling pathways. Journal of Agriculture and Food Chemistry 2013, 61, 1509-1520.

146. Osorio-Fuentealba, C.; Contreras-Ferrat, A. E.; Altamirano, F.; Espinosa, A.; Li, Q.; Niu, W.; Lavandero, S.; Klip, A.; Jaimovich, E., Electrical stimuli release ATP to increase GLUT4 translocation and glucose uptake via PI3K $\gamma$-Akt-AS160 in skeletal muscle cells. Diabetes 2013, 62, 1519-1526. 
147. Huang, Z.; Xie, X., Chemerin induces insulin resistance in $\mathrm{C} 2 \mathrm{C} 12$ cells through nuclear factor-kappaB pathway-mediated inflammatory reaction. Chinese J. Cell. Mol. Immun. 2015, 31, 725-729.

148. Sun, Q.; Peng, Y.; Qi, W.; Kim, Y.; Clark, J. M.; Kim, D.; Park, Y., Permethrin decreased insulin-stimulated AKT phosphorylation dependent on extracellular signalregulated kinase-1 (ERK), but not AMP-activated protein kinase alpha (AMPKalpha), in C2C12 myotubes. Food Chem. Toxicol. 2017, 109, 95-101.

149. Kim, S.; Go, G. W.; Imm, J. Y., Promotion of glucose uptake in C2C12 myotubes by Cereal Flavone Tricin and its underlying molecular mechanism. J. Agric. Food Chem. 2017, 65, 3819-3826.

150. Sun, C.; Zhang, F.; Ge, X.; Yan, T.; Chen, X.; Shi, X.; Zhai, Q., SIRT1 improves insulin sensitivity under insulin-resistant conditions by repressing PTP1B. Cell Metab. 2007, 6, 307-319.

151. Sun, Q.; Qi, W.; Xiao, X.; Yang, S. H.; Kim, D.; Yoon, K. S.; Clark, J. M.; Park, Y., Imidacloprid promotes high fat diet-induced adiposity in female C57BL/6J mice and enhances adipogenesis in 3T3-L1 adipocytes via the AMPKalpha-mediated pathway. $J$. Agric. Food Chem. 2017, 65, 6572-6581.

152. Azay-Milhau, J.; Ferrare, K.; Leroy, J.; Aubaterre, J.; Tournier, M.; Lajoix, A. D.; Tousch, D., Antihyperglycemic effect of a natural chicoric acid extract of chicory (Cichorium intybus L.): A comparative in vitro study with the effects of caffeic and ferulic acids. J. Ethnopharmacol. 2013, 150, 755-760.

153. García-Vicente, S.; Yraola, F.; Marti, L.; González-Muñoz, E.; García-Barrado, M. J.; Cantó, C.; Abella, A.; Bour, S.; Artuch, R.; Sierra, C.; Brandi, N.; Carpéné, C.; Moratinos, J.; Camps, M.; Palacín, M.; Testar, X.; Gumà, A.; Albericio, F.; Royo, M.; Mian, A.; Zorzano, A., Oral insulin-mimetic compounds that act independently of insulin. Diabetes 2007, 56, 486-493.

154. Wan, Z.; Root-McCaig, J.; Castellani, L.; Kemp, B. E.; Steinberg, G. R.; Wright, D. C., Evidence for the role of AMPK in regulating PGC-1 alpha expression and mitochondrial proteins in mouse epididymal adipose tissue. Obesity 2014, 22, 730-738.

155. Canto, C.; Auwerx, J., PGC-1alpha, SIRT1 and AMPK, an energy sensing network that controls energy expenditure. Curr. Opin. Lipidol. 2009, 20, 98-105.

156. Eid, H. M.; Vallerand, D.; Muhammad, A.; Durst, T.; Haddad, P. S.; Martineau, L. C., Structural constraints and the importance of lipophilicity for the mitochondrial uncoupling activity of naturally occurring caffeic acid esters with potential for the treatment of insulin resistance. Biochem. Pharmacol. 2010, 79, 444-454. 
157. Lee, E. S.; Uhm, K.; Lee, Y. M.; Han, M.; Lee, M.; Park, J. M.; Suh, P.; Park, S.;

Kim, H. S., CAPE (caffeic acid phenethyl ester) stimulates glucose uptake through

AMPK (AMP-activated protein kinase) activation in skeletal muscle cells. Biochem.

Biophys. Res. Commun. 2007, 361, 854-858. 